\title{
Internet-based cognitive and behavioural therapies for post- traumatic stress disorder (PTSD) in adults (Review)
}

Lewis C, Roberts NP, Bethell A, Robertson L, Bisson JI

Lewis C, Roberts NP, Bethell A, Robertson L, Bisson JI.

Internet-based cognitive and behavioural therapies for post-traumatic stress disorder (PTSD) in adults.

Cochrane Database of Systematic Reviews 2018, Issue 12. Art. No.: CD011710.

DOI: 10.1002/14651858.CD011710.pub2.

www.cochranelibrary.com 
TABLE OF CONTENTS

HEADER

ABSTRACT

PLAIN LANGUAGE SUMMARY

SUMMARY OF FINDINGS

BACKGROUND

OBJECTIVES

METHODS

RESULTS

Figure 1.

Figure 2.

Figure 3.

DISCUSSION

AUTHORS' CONCLUSIONS

ACKNOWLEDGEMENTS

REFERENCES

CHARACTERISTICS OF STUDIES

DATA AND ANALYSES

Analysis 1.1. Comparison 1: Internet-based cognitive and behavioural therapy (I-C/BT) versus wait list (WL), Outcome 1: Severity of PTSD symptoms (post-treatment)

Analysis 1.2. Comparison 1: Internet-based cognitive and behavioural therapy (I-C/BT) versus wait list (WL), Outcome 2: Severity of PTSD symptoms (follow-up $<6$ months)

Analysis 1.3. Comparison 1: Internet-based cognitive and behavioural therapy (I-C/BT) versus wait list (WL), Outcome 3: Dropouts

Analysis 1.4. Comparison 1: Internet-based cognitive and behavioural therapy (I-C/BT) versus wait list (WL), Outcome 4: Diagnosis of PTSD after treatment

Analysis 1.5. Comparison 1: Internet-based cognitive and behavioural therapy (I-C/BT) versus wait list (WL), Outcome 5: Severity of depression (post-treatment)

Analysis 1.6. Comparison 1: Internet-based cognitive and behavioural therapy (I-C/BT) versus wait list (WL), Outcome 6: Severity of depression (follow-up $<6$ months)

Analysis 1.7. Comparison 1: Internet-based cognitive and behavioural therapy (I-C/BT) versus wait list (WL), Outcome 7: Severity of anxiety symptoms (post-treatment)

Analysis 1.8. Comparison 1: Internet-based cognitive and behavioural therapy (I-C/BT) versus wait list (WL), Outcome 8: Severity of anxiety symptoms (follow-up $<6$ months)

Analysis 1.9. Comparison 1: Internet-based cognitive and behavioural therapy (I-C/BT) versus wait list (WL), Outcome 9: Quality of Life (post-treatment)

Analysis 2.1. Comparison 2: Internet-based cognitive and behavioural therapy (I-C/BT) versus I-non-C/BT, Outcome 1: Severity of PTSD symptoms (post-treatment)

Analysis 2.2. Comparison 2: Internet-based cognitive and behavioural therapy (I-C/BT) versus I-non-C/BT, Outcome 2: Severity of PTSD symptoms (follow-up $<6$ months)

Analysis 2.3. Comparison 2: Internet-based cognitive and behavioural therapy (I-C/BT) versus I-non-C/BT, Outcome 3: Severity of PTSD symptoms (follow-up 6-12 months)

Analysis 2.4. Comparison 2: Internet-based cognitive and behavioural therapy (I-C/BT) versus I-non-C/BT, Outcome 4: Dropouts

Analysis 2.5. Comparison 2: Internet-based cognitive and behavioural therapy (I-C/BT) versus I-non-C/BT, Outcome 5: Severity of depressive symptoms (post-treatment)

Analysis 2.6. Comparison 2: Internet-based cognitive and behavioural therapy (I-C/BT) versus I-non-C/BT, Outcome 6: Severity of depressive symptoms (follow-up $<6$ months)

Analysis 2.7. Comparison 2: Internet-based cognitive and behavioural therapy (I-C/BT) versus I-non-C/BT, Outcome 7: Severity of depressive symptoms (follow-up 6-12 months)

Analysis 2.8. Comparison 2: Internet-based cognitive and behavioural therapy (I-C/BT) versus I-non-C/BT, Outcome 8: Severity of anxiety symptoms (post-treatment)

Analysis 2.9. Comparison 2: Internet-based cognitive and behavioural therapy (I-C/BT) versus I-non-C/BT, Outcome 9: Severity of anxiety symptoms (follow-up $<6$ months) 
Analysis 2.10. Comparison 2: Internet-based cognitive and behavioural therapy (I-C/BT) versus I-non-C/BT, Outcome 10:

Severity of anxiety symptoms (follow-up 6-12 months)

APPENDICES

WHAT'S NEW

HISTORY

CONTRIBUTIONS OF AUTHORS

DECLARATIONS OF INTEREST

SOURCES OF SUPPORT 
[Intervention Review]

\section{Internet-based cognitive and behavioural therapies for post-traumatic stress disorder (PTSD) in adults}

Catrin Lewis ${ }^{1}$, Neil P Roberts 1,2 , Andrew Bethell ${ }^{3}$, Lindsay Robertson 4 , Jonathan I Bisson ${ }^{1}$

1Division of Psychological Medicine and Clinical Neurosciences, Cardiff University School of Medicine, Cardiff, UK. 2Psychology \& Psychological Therapies Directorate, Cardiff and Vale University Health Board, Cardiff, UK. 3 Institute of Psychology, Health and Society, University of Liverpool, Liverpool, UK. ${ }^{4}$ Cochrane Common Mental Disorders, University of York, York, UK

Contact address: Catrin Lewis, LewisCE7@cf.ac.uk.

Editorial group: Cochrane Common Mental Disorders Group.

Publication status and date: Edited (no change to conclusions), published in Issue 5, 2020.

Citation: Lewis C, Roberts NP, Bethell A, Robertson L, Bisson JI. Internet-based cognitive and behavioural therapies for posttraumatic stress disorder (PTSD) in adults. Cochrane Database of Systematic Reviews 2018, Issue 12. Art. No.: CD011710. DOI: 10.1002/14651858.CD011710.pub2.

Copyright @ 2018 The Cochrane Collaboration. Published by John Wiley \& Sons, Ltd.

\section{A B S T R A C T}

\section{Background}

Therapist-delivered trauma-focused psychological therapies are an effective treatment for post-traumatic stress disorder (PTSD). These have become the accepted first-line treatments for the disorder. Despite the established evidence-base for these therapies, they are not always widely available or accessible. Many barriers limit treatment uptake, such as the limited number of qualified therapists to deliver the interventions, cost, and compliance issues, such as time off work, childcare, and transportation, associated with the need to attend weekly appointments. Delivering cognitive behavioural therapy (CBT) on the Internet is an effective and acceptable alternative to therapistdelivered treatments for anxiety and depression. However, fewer Internet-based therapies have been developed and evaluated for PTSD, and uncertainty surrounds the efficacy of Internet-based cognitive and behavioural therapy (I-C/BT) for PTSD.

\section{Objectives}

To assess the effects of I-C/BT for PTSD in adults.

\section{Search methods}

We searched the Cochrane Common Mental Disorders Group's Specialised Register (CCMDCTR) to June 2016 and identified four studies meeting the inclusion criteria. The CCMDCTR includes relevant randomised controlled trials (RCT) from MEDLINE, Embase, and PsycINFO. We also searched online clinical trial registries and reference lists of included studies, and contacted researchers in the field to identify additional and ongoing studies. We ran an update search on 1 March 2018, and identified four additional completed studies, which we added to the analyses along with two that were previously awaiting classification.

\section{Selection criteria}

We searched for RCTs of I-C/BT compared to face-to-face or Internet-based psychological treatment, psychoeducation, wait list or care as usual. We included studies of adults (aged over 16 years or over), in which at least $70 \%$ of the participants met the diagnostic criteria for PTSD, according to the Diagnostic and Statistical Manual (DSM) or the International Classification of Diseases (ICD).

\section{Data collection and analysis}

We entered data into Review Manager 5 software. We analysed categorical outcomes as risk ratios (RRs), and continuous outcomes as mean differences (MD) or standardised mean differences (SMDs), with 95\% confidence intervals (CI). We pooled data with a fixed-effect metaanalysis, except where heterogeneity was present, in which case we used a random-effects model. Two review authors independently 
assessed the included studies for risk of bias; any conflicts were discussed with a third author, with the aim of reaching a unanimous decision.

\section{Main results}

We included 10 studies with 720 participants in the review. Eight of the studies compared I-C/BT delivered with therapist guidance to a wait list control. Two studies compared guided I-C/BT with I-non-C/BT. There was considerable heterogeneity among the included studies.

Very low-quality evidence showed that, compared with wait list, I-C/BT may be associated with a clinically important reduction in PTSD post-treatment (SMD $-0.60,95 \% \mathrm{Cl}-0.97$ to -0.24 ; studies $=8$, participants $=560$ ). However, there was no evidence of a difference in PTSD symptoms when follow-up was less than six months (SMD $-0.43,95 \% \mathrm{Cl}-1.41$ to 0.56 ; studies $=3$, participants $=146$ ). There may be little or no difference in dropout rates between the I-C/BT and wait list groups (RR 1.39, 95\% Cl 1.03 to 1.88; studies $=8$, participants $=585$; lowquality evidence). I-C/BT was no more effective than wait list at reducing the risk of a diagnosis of PTSD after treatment (RR $0.53,95 \% \mathrm{Cl}$ 0.28 to 1.00 ; studies $=1$, participants $=62$; very low-quality evidence). I-C/BT may be associated with a clinically important reduction in symptoms of depression both post-treatment (SMD $-0.61,95 \% \mathrm{Cl}-1.17$ to -0.05 ; studies $=5$, participants $=425$; very low-quality evidence). Very low-quality evidence also suggested that I-C/BT may be associated with a clinically important reduction in symptoms of anxiety posttreatment (SMD $-0.67,95 \% \mathrm{Cl}-0.98$ to -0.36 ; studies $=4$, participants $=305$ ), and at follow-up less than six months $(\mathrm{MD}-12.59,95 \% \mathrm{Cl}-$ 20.74 to -4.44 ; studies $=1$, participants $=42$; very low-quality evidence). The effects of I-C/BT on quality of life were uncertain (SMD 0.60 , $95 \% \mathrm{Cl} 0.08$ to 1.12 ; studies $=2$, participants $=221$; very low-quality evidence) .

Two studies found no difference in PTSD symptoms between the I-C/BT and I-non-C/BT groups when measured post-treatment (SMD -0.08, $95 \% \mathrm{Cl}-0.52$ to 0.35 ; studies $=2$, participants $=82$; very low-quality evidence), or when follow-up was less than six months (SMD $0.08,95 \% \mathrm{Cl}$ -0.41 to 0.57 ; studies $=2$, participants $=65$; very low-quality evidence). However, those who received I-C/BT reported their PTSD symptoms were better at six- to 12 -month follow-up ( $\mathrm{MD}-8.83,95 \% \mathrm{Cl}-17.32$ to -0.34 ; studies $=1$, participants $=18$; very low-quality evidence). Two studies found no difference in depressive symptoms between the I-C/BT and I-non-C/BT groups when measured post-treatment (SMD $0.12,95 \% \mathrm{Cl}-0.78$ to 0.54 ; studies $=2$, participants $=84$; very low-quality evidence) or when follow-up was less than six months (SMD $0.20,95 \% \mathrm{Cl}-0.31$ to 0.71 ; studies $=2$, participants $=61$; very low-quality evidence). However, those who received I-C/BT reported their depressive symptoms were better at six- to 12 -month follow-up ( $\mathrm{MD}-8.34,95 \% \mathrm{Cl}-15.83$ to -0.85 ; studies $=1$, participants $=18$; very lowquality evidence). Two studies found no difference in symptoms of anxiety between the I-C/BT and I-non-C/BT groups when measured post-treatment (SMD $0.08,95 \% \mathrm{Cl}-0.78$ to 0.95 ; studies $=2$, participants $=74$; very low-quality evidence) or when follow-up was less than six months (SMD $-0.16,95 \% \mathrm{Cl}-0.67$ to 0.35 ; studies = 2, participants $=60$; very low-quality evidence). However, those who received I-C/BT reported their symptoms of anxiety were better at six- to 12 -month follow-up (MD $-8.05,95 \% \mathrm{Cl}-15.20$ to -0.90 ; studies $=1$, participants = 18; very low-quality evidence).

None of the included studies reported on cost-effectiveness or adverse events.

\section{Authors' conclusions}

While the review found some beneficial effects of I-C/BT for PTSD, the quality of the evidence was very low due to the small number of included trials. Further work is required to: establish non-inferiority to current first-line interventions, explore mechanisms of change, establish optimal levels of guidance, explore cost-effectiveness, measure adverse events, and determine predictors of efficacy and dropout.

\section{PLAIN LANGUAGE SUMMARY}

\section{Internet-based cognitive and behavioural therapies for post-traumatic stress disorder (PTSD)}

\section{Why was this review important?}

Post-traumatic stress disorder, or PTSD, is a common mental illness that can occur after a serious traumatic event. Symptoms include reexperiencing the trauma as nightmares, flashbacks, and distressing thoughts; avoiding reminders of the traumatic event; experiencing negative changes to thoughts and mood; and hyperarousal, which includes feeling on edge, being easily startled, feeling angry, having difficulties sleeping, and problems concentrating. PTSD can be treated effectively with talking therapies that focus on the trauma. Some of the most effective therapies are those based on cognitive behavioural therapy (CBT). Unfortunately, there are a limited number of qualified therapists who are able to deliver these therapies. There are also other factors that limit access to treatment, such as taking time off work to attend appointments, and transportation issues.

An alternative is to deliver psychological therapy on the Internet, with or without guidance from a therapist. Internet-based cognitive and behavioural therapies (I-C/BT) have received a great deal of attention, and are now used routinely to treat depression and anxiety. There have been fewer studies of I-C/BT for PTSD, and we do not yet know whether they are effective.

\section{Who will be interested in this review?}

- People with PTSD and their loved ones.

- Professionals working in mental health services. 
- General practitioners.

- Commissioners.

\section{What questions did this review try to answer?}

In adults with PTSD, we tried to find out if I-C/BT:

- was more effective than no therapy (waiting list);

- was as effective as psychological therapies delivered by a therapist;

- was more effective than other psychological therapies delivered online; or

- was more effective than education about the condition delivered online, at reducing symptoms of PTSD, and improving quality of life; or - was cost effective, compared to face-to-face therapy?

\section{Which studies were included in the review?}

We searched for randomised controlled trials (clinical studies where people are randomly put into one of two or more treatment groups) that examined I-C/BT for adults with PTSD, published between 1970 and 2 March 2018.

We included 10 studies with 720 participants.

\section{What did the evidence from the review tell us?}

- Very low-quality evidence from eight studies found that I-C/BT was more effective than no therapy (waiting list) at reducing symptoms of PTSD.

- Very low-quality evidence from two studies found no significant difference between I-C/BT and another type of psychological therapy delivered online.

-We found no studies that compared I-C/BT to psychological therapy delivered by a therapist, or education about the condition delivered online.

-We found no evidence to tell us whether people who received I-C/BT felt it was an acceptable treatment, or whether it was effective in improving quality of life.

-We found no studies that reported on the cost effectiveness of I-C/BT.

\section{What should happen next?}

The current evidence base is small. More studies are needed to decide if I-C/BT should be used routinely for the treatment of PTSD. 


\section{S U M MARY OF FINDINGS}

Summary of findings 1. Internet-based cognitive and behavioural therapy (I-C/BT) compared to wait list for post-traumatic stress disorder (PTSD) in adults

\section{I-C/BT compared to wait list for PTSD in adults}

Patient or population: adults with PTSD

Setting:

Intervention: I-C/BT

Comparison: wait list

\begin{tabular}{|c|c|c|c|c|c|c|}
\hline \multirow[t]{2}{*}{ Outcomes } & \multicolumn{2}{|c|}{ Anticipated absolute effects* $(95 \% \mathrm{Cl})$} & \multirow{2}{*}{$\begin{array}{l}\text { Relative ef- } \\
\text { fect } \\
(95 \% \mathrm{CI})\end{array}$} & \multirow{2}{*}{$\begin{array}{l}\text { № of partici- } \\
\text { pants } \\
\text { (studies) }\end{array}$} & \multirow{2}{*}{$\begin{array}{l}\text { Certainty of } \\
\text { the evidence } \\
\text { (GRADE) }\end{array}$} & \multirow[t]{2}{*}{ Comments } \\
\hline & Risk with wait list & Risk with I-C/BT & & & & \\
\hline $\begin{array}{l}\text { Severity of PTSD symptoms (mea- } \\
\text { sured with the IES-R, CAPS-5, PCL- } \\
\text { CPSS-I and PDS; higher score = } \\
\text { worse outcome) }\end{array}$ & $\begin{array}{l}\text { The mean severity of PTSD } \\
\text { symptoms (post-treatment) } \\
\text { was } 0\end{array}$ & $\begin{array}{l}\text { SMD } 0.6 \text { lower } \\
\text { ( } 0.97 \text { lower to } 0.24 \text { lower) }\end{array}$ & - & $\begin{array}{l}560 \\
\text { (8 RCTs) }\end{array}$ & $\begin{array}{l}\oplus \ominus \ominus \ominus \\
\text { Very low } a, b\end{array}$ & - \\
\hline \multicolumn{7}{|l|}{ Follow-up: post-treatment } \\
\hline \multirow[t]{2}{*}{ Dropouts } & \multicolumn{2}{|l|}{ Study population } & \multirow{2}{*}{$\begin{array}{l}\text { RR } 1.39 \\
\text { (1.03 to } 1.88)\end{array}$} & \multirow{2}{*}{$\begin{array}{l}585 \\
\text { (8 RCTs) }\end{array}$} & \multirow{2}{*}{$\begin{array}{l}\oplus \oplus \ominus \ominus \\
\text { Low } a\end{array}$} & \multirow[t]{2}{*}{-} \\
\hline & 186 per 1000 & $\begin{array}{l}258 \text { per } 1000 \\
(192 \text { to } 350)\end{array}$ & & & & \\
\hline \multirow[t]{2}{*}{ Diagnosis of PTSD after treatment } & Study population & & \multirow{2}{*}{$\begin{array}{l}\text { RR } 0.53 \\
(0.28 \text { to } 1.00)\end{array}$} & \multirow{2}{*}{$\begin{array}{l}62 \\
(1 \mathrm{RCT})\end{array}$} & \multirow{2}{*}{$\begin{array}{l}\oplus \ominus \ominus \ominus \\
\text { Very low } \mathrm{c}, \mathrm{d}\end{array}$} & \multirow[t]{2}{*}{-} \\
\hline & 548 per 1000 & $\begin{array}{l}291 \text { per } 1000 \\
(154 \text { to } 548)\end{array}$ & & & & \\
\hline $\begin{array}{l}\text { Severity of depressive symptoms } \\
\text { (measured with the BDI, PHQ and } \\
\text { CES-D; higher score = worse out- } \\
\text { come) }\end{array}$ & $\begin{array}{l}\text { The mean depression (post- } \\
\text { treatment) was } 0\end{array}$ & $\begin{array}{l}\text { SMD } 0.61 \text { lower } \\
\text { (1.17 lower to } 0.05 \text { lower) }\end{array}$ & - & $\begin{array}{l}425 \\
\text { (5 RCTs) }\end{array}$ & $\begin{array}{l}\oplus \ominus \odot \odot \\
\text { Very low b,e }\end{array}$ & - \\
\hline \multicolumn{7}{|l|}{ Follow-up: post-treatment } \\
\hline $\begin{array}{l}\text { Severity of anxiety symptoms } \\
\text { (measured with the BAI and GAD-7; } \\
\text { higher score = worse outcome) }\end{array}$ & $\begin{array}{l}\text { The mean anxiety (post- } \\
\text { treatment) was } 0\end{array}$ & $\begin{array}{l}\text { SMD } 0.67 \text { lower } \\
\text { (0.98 lower to } 0.36 \text { lower) }\end{array}$ & - & $\begin{array}{l}305 \\
\text { (4 RCTs) }\end{array}$ & $\begin{array}{l}\oplus \odot \odot \ominus \\
\text { Very low } \mathrm{f}, \mathrm{g}\end{array}$ & - \\
\hline
\end{tabular}




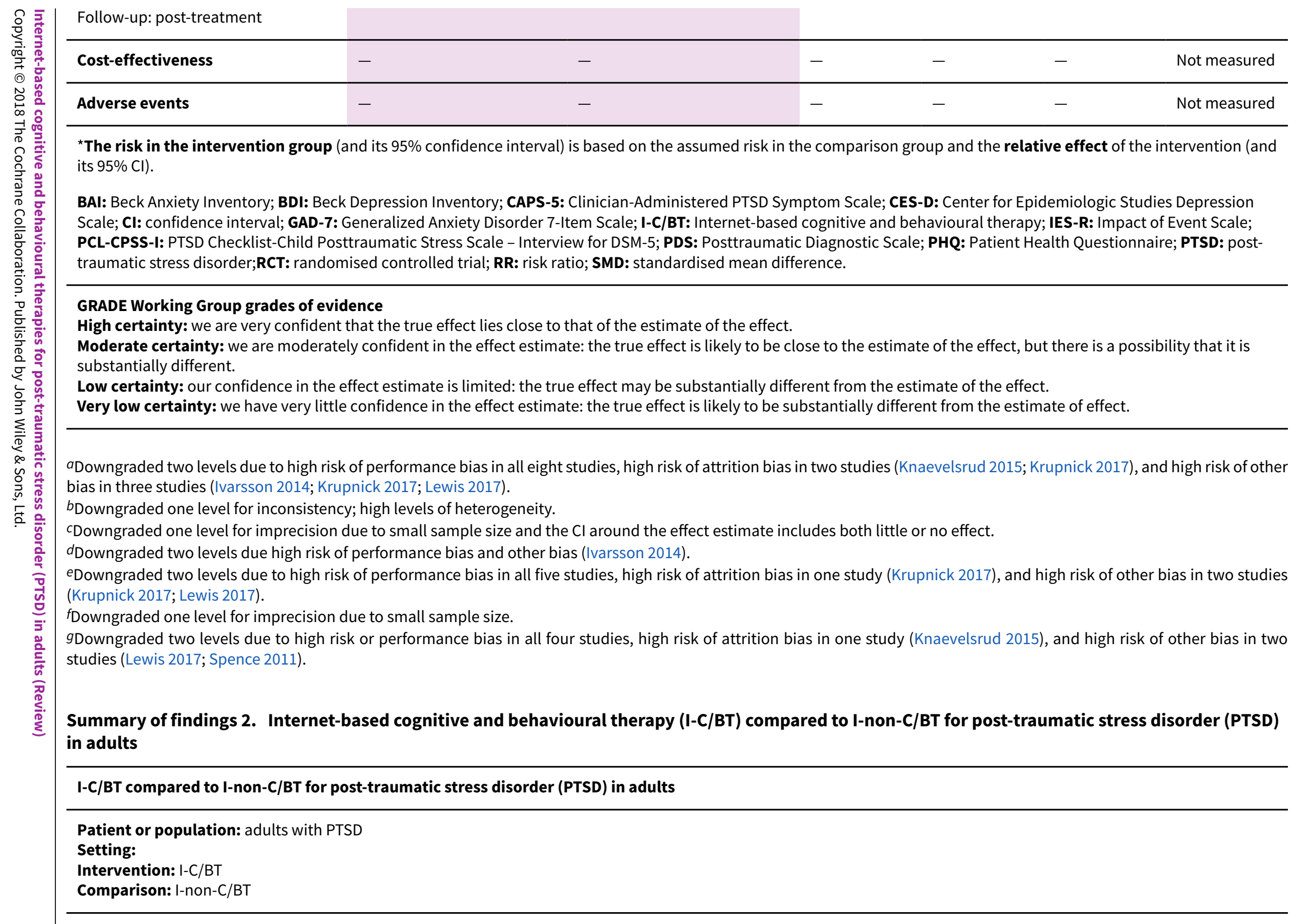

BAI: Beck Anxiety Inventory; BDI: Beck Depression Inventory; CAPS-5: Clinician-Administered PTSD Symptom Scale; CES-D: Center for Epidemiologic Studies Depression (

High certainty: we are very confident that the true effect lies close to that of the estimate of the effect.

substantially different.

Low certainty: our confidence in the effect estimate is limited: the true effect may be substantially different from the estimate of the effect.

a Downgraded two levels due to high risk of performance bias in all eight studies, high risk of attrition bias in two studies (Knaevelsrud 2015; Krupnick 2017), and high risk of other bias in three studies (Ivarsson 2014; Krupnick 2017; Lewis 2017).

fDowngraded one level for imprecision due to small sample size.

gDowngraded two levels due to high risk or performance bias in all four studies, high risk of attrition bias in one study (Knaevelsrud 2015), and high risk of other bias in two studies (Lewis 2017; Spence 2011).

Summary of findings 2. Internet-based cognitive and behavioural therapy (I-C/BT) compared to I-non-C/BT for post-traumatic stress disorder (PTSD) in adults 


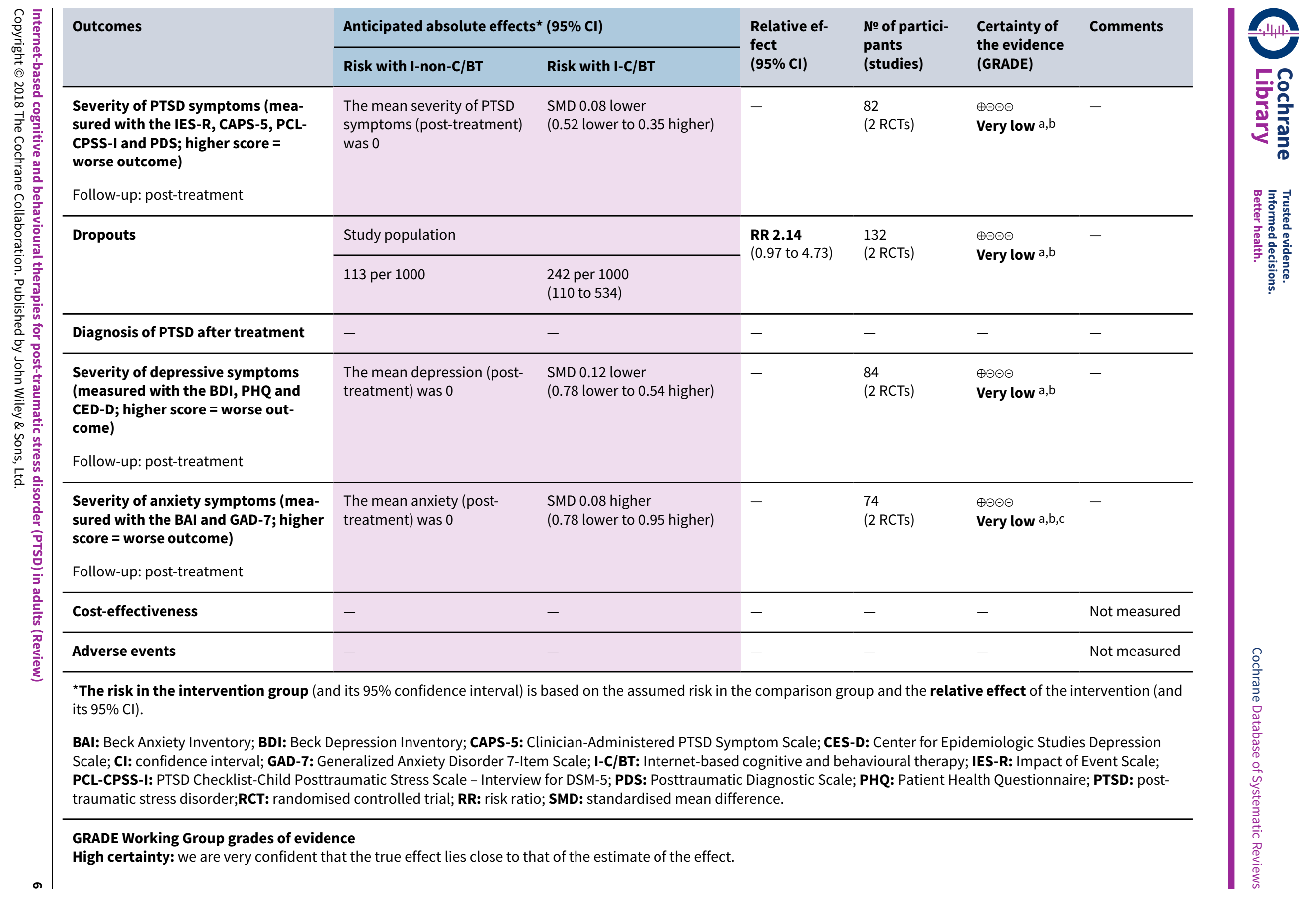


Moderate certainty: we are moderately confident in the effect estimate: the true effect is likely to be close to the estimate of the effect, but there is a possibility that it is substantially different.

Low certainty: our confidence in the effect estimate is limited: the true effect may be substantially different from the estimate of the effect.

Very low certainty: we have very little confidence in the effect estimate: the true effect is likely to be substantially different from the estimate of effect.

aDowngraded two levels due to high risk of performance bias due to lack of blinding participants and personnel in both studies (Littleton 2016; Litz 2007), high risk of detection bias due to lack of blinding outcome assessors in one study (Littleton 2016), and high risk of attrition bias and other bias in one study (Litz 2007).

bDowngraded two levels for imprecision due to small sample size and the $\mathrm{Cl}$ of the effect estimate includes both little or no effect.

cDowngraded one level for inconsistency due to high levels of heterogeneity. 


\section{B A C K G R O U N D}

\section{Description of the condition}

Post-traumatic stress disorder (PTSD) is characterised by the development of distressing psychological symptoms following exposure to a traumatic event (APA 2013). Exposure can involve direct personal experience, or can occur when witnessing a traumatic event happening to another person. Qualifying traumas are "of an exceptionally threatening or catastrophic nature, which is likely to cause pervasive distress in almost anyone" (WHO 1992). PTSD does not represent a valid diagnosis after life-events such as divorce or losing a job.

Diagnostic symptoms of PTSD include re-experiencing the trauma as upsetting thoughts, nightmares, or flashbacks; avoiding thoughts about the trauma or reminders of it; negative alterations in mood or cognitions, including difficulties recalling the trauma and constricted affect; and heightened physiological arousal, which can manifest as hypervigilance, exaggerated startle responses, and difficulties concentrating or sleeping. Diagnosis is dependent on symptoms that cause clinically significant distress and impairment to the person's capacity to work, socialise, or function in other important domains (APA 2013).

PTSD is a common disorder that imposes a significant personal and societal burden. Epidemiological research suggests that $60 \%$ of men and $50 \%$ of women experience at least one PTSD-qualifying traumatic event through the course of their lives (Kessler 1995). Lifetime prevalence of PTSD in Europe has been estimated at between 1.9\% (Alonso 2014) and 11\% (Ferry 2010); one largescale survey in Australia reported a 12-month prevalence of $1.33 \%$ (Creamer 2001), and a survey in Mexico revealed a lifetime prevalence rate of $11.2 \%$ (Norris 2003). Approximately $50 \%$ of people diagnosed with PTSD recover within two years, while about a third continue to meet criteria for diagnosis six years later (Kessler 1995).

There is substantial comorbidity between PTSD and other psychiatric disorders. One large-scale survey in the USA revealed the coexistence of at least one additional disorder in $88.3 \%$ of men and $79 \%$ of women with a history of PTSD (Kessler 1995). One UK survey found that people with PTSD were twice as likely as people without PTSD to have at least one other comorbid mood, anxiety, or substance-use disorder (NICTT 2008). Risk factors for the development of PTSD include pretrauma factors, such as psychiatric history and traumatic events; peritrauma factors, such as trauma severity and dissociation; and post-trauma factors, such as poor social support (Brewin 2000; Ozer 2003).

\section{Description of the intervention}

International guidelines for the treatment of PTSD converge on the recommendation of trauma-focused psychological therapies, including trauma-focused cognitive behavioural therapy (CBT), as effective treatments for the disorder (ACPMH 2007; APA 2004; NCCMH 2005). These are currently the treatments of choice for people with PTSD, but evidence is insufficient to support the use of pharmacotherapy in isolation, or the provision of nontrauma-focused psychological therapies, such as CBT without a trauma focus, supportive therapy, non-directive counselling, psychodynamic therapy, and present-centred therapy (NCCMH 2005). A shortage of suitably qualified and trained therapists who can deliver these interventions has prompted interest in new interventions that place less reliance on therapist time (Lewis 2013).

Internet-based cognitive and behavioural therapies (I-C/BT) deliver treatment protocols online using multi-media delivery methods (Cuijpers 2008). Therapists provide sufficient instruction to teach coping skills, or bring about improvement in target symptoms with limited therapist input (Spek 2007). I-C/BT programmes have been developed and implemented for a range of disorders, with the aim of reducing healthcare expenditures and broadening access to psychological therapies (Lewis 2010). The content of existing therapies is not usually altered, deviating from traditional psychological treatment only in terms of method of delivery (Cuijpers 2010).

The distinction between I-C/BT and online psychoeducation must be clear. Although the two overlap in content, psychoeducation aims to increase patient knowledge, while I-C/BT aims to teach skills and techniques that can be used to overcome specific symptoms (IAPT 2010). I-C/BT programmes are usually based on existing protocols, and share many common features (Andersson 2005). Most start treatment with psychoeducation, and then present the rationale for CBT-based treatment (IAPT 2010). These programmes incorporate cognitive techniques with the aim of identifying and modifying unhelpful patterns of cognition (Newman 2003). Usually, behavioural components are included also; for PTSD, they generally encompass imaginal exposure (which creates a narrative of the trauma memory and engages in repeated exposure to it), and in vivo exposure (which involves gradual, repeated exposure to feared or avoided situations; Lewis 2012). Most Internet-based self-help programmes conclude with a section on relapse prevention that focuses on staying well, recognising signs of relapse, and offering advice on what to do if problems recur (Gega 2004).

Many different types of I-C/BT have been developed; they can be distinguished on the basis of the level of therapist assistance provided. First, I-C/BT can be delivered with regular assistance from a highly engaged specialist who provides input and feedback on homework, which acts as a direct analogue to specialty care. Second, I-C/BT can be delivered by a non-specialist mental health professional who briefly introduces the programme and briefly intervenes to check on progress, often by telephone or by e-mail. Finally, I-C/BT can adopt a pure self-help approach, in which the participant is the sole agent of change, and no therapist assistance is provided.

\section{How the intervention might work}

Internet interventions may take a cognitive therapy (CT) or a behavioural therapy (BT) approach, but most are based on CBT (Andersson 2009). The core premise of CBT for PTSD is that fear conditioning and maladaptive cognitions contribute to emotional distress and problematic behaviours. Several disorder-specific CBT protocols have been developed, and the approach involves a collaborative problem-solving process, aimed at exploring and challenging unhelpful cognitions, and modifying problematic behaviours. Many I-C/BT programmes for PTSD have taken a trauma-focused CBT approach, which relies on general cognitive and behavioural techniques, with additional components aimed at addressing problematic thoughts and behaviours arising from the traumatic event itself. 
Face-to-face trauma-focused CBT is evidence based, and protocols draw on four core components: psychoeducation, anxiety management, exposure, and cognitive restructuring (Bisson 2013). These components are incorporated into Internet adaptations of the intervention (Lange 2003). Psychoeducation usually provides the starting point; anxiety management, exposure, and cognitive work are then gradually introduced (NCCMH 2005). Anxiety management techniques strengthen the individual's ability to cope with PTSD symptoms, recollection of traumatic memories, and the therapeutic process. Anxiety management may include breathing techniques, progressive muscle relaxation, or forms of guided imagery. Exposure plays an important role in many trauma-focused CBT protocols and may be carried out in vivo (real life) or imaginally (Bryant 2003). It is common for both techniques to be used in the treatment of people with PTSD, to target internally and externally feared stimuli (Creamer 2004). The trauma memory itself is often the primary feared stimulus, and exposure to the memory is carried out imaginally. The rationale for the use of imaginal exposure varies according to the specific trauma-focused CBT protocol applied. Imaginal exposure is based on principles of habituation (reduction of anxiety after prolonged exposure), information processing (reevaluation of old information and incorporation of new information into the trauma memory), or both (Foa 2008). In vivo exposure encourages the person to confront feared situations in real life, and cognitive work seeks to identify and modify unhelpful thoughts by testing and challenging self-held beliefs (Foa 2007; Wilson 2012).

\section{Why it is important to do this review}

PTSD causes clinically significant distress and impacts functioning (APA 2013). Therefore, it is important to develop effective interventions. Several systematic reviews of interventions for PTSD have been published in the Cochrane Library. Bisson 2013 and Bradley 2005 described fairly robust evidence for traumafocused CBT and eye movement desensitisation and reprocessing (EMDR) as treatments for chronic PTSD, with emerging evidence for some non-trauma-focused CBT interventions and trauma-focused CBT-based group interventions. Other Cochrane reviews have considered single-session psychological 'debriefing' (Rose 2002), and multiple-session early psychological interventions to prevent PTSD (Roberts 2009); and early psychological therapies (Roberts 2010), pharmacological treatments (Stein 2006), combined pharmacotherapy and psychological therapies (Hetrick 2010), and psychological therapies to treat PTSD in children and adolescents (Gillies 2012). Internet-based self-help represents an increasingly popular way of delivering psychological therapy, but its use for the treatment of people with PTSD has lagged behind that for other disorders (Lewis 2012). Internet-based cognitive and behavioural therapies for depression and anxiety have received significant attention, and numerous systematic reviews and meta-analyses have explored the efficacy of these interventions (e.g. Cuijpers 2008; Spek 2007). Positive findings, and the potential for these Internet-based treatments to broaden access to psychological therapy and reduce costs, have spurred on the development and evaluation of similar interventions for a wide range of mental health problems. This has led to a proliferation of studies evaluating the efficacy of I-C/BT for PTSD, deeming it a good time to conduct a Cochrane Review on this topic.

Two Cochrane Reviews are related to the current work, including the review of psychological therapies for PTSD, which did not include I-C/BT treatments (Bisson 2013), and a review of media- delivered CBT for anxiety disorders in adults, which excluded PTSD, as it was set to be separated from anxiety disorders in the Diagnostic and Statistical Manual of Mental Disorders, Fifth Edition (DSM-5), and the International Classification of Diseases, 11th Edition (ICD-11; Mayo-Wilson 2013). A third Cochrane Review of IC/BT for anxiety disorders included PTSD as an eligible diagnosis, but excluded interventions provided without therapist assistance, and interventions that included face-to-face therapist assistance; the stringent inclusion criteria resulted in inclusion of only one trial of I-C/BT for PTSD (Olthuis 2015). Therefore, there still is the need to summarise the evidence base for I-C/BT, which is unguided, or uses minimal face-to-face guidance.

\section{O B J E C T IVES}

To assess the effects of I-C/BT for PTSD in adults.

\section{METHODS}

\section{Criteria for considering studies for this review}

\section{Types of studies}

Randomised controlled trials (RCTs), randomised cross-over trials, and cluster-randomised trials. We only used data from the first randomisation period of cross-over trials to avoid a carry-over effect. We did not use sample size or publication status to determine whether a study should be included. We included studies published in all languages.

\section{Types of participants}

\section{Participant characteristics}

Adults, 16 years of age or older. We did not consider I-C/BT interventions for children under the age of 16 years for this review. We applied no restrictions on gender or ethnicity.

\section{Diagnosis}

Participants had traumatic stress symptoms, and at least $70 \%$ of people in any given study were required to meet diagnostic criteria for PTSD according to the Diagnostic and Statistical Manual of Mental Disorders (DSM-III; APA 1980), DSM-IIIR (APA 1987), DSM-IV (APA 2000), DSM-5 (APA 2013), International Statistical Classification of Diseases and Related Health Problems (ICD-9; WHO 1979), or ICD-10 (WHO 1992), assessed by clinical interview or a validated questionnaire. We included studies regardless of the index trauma, severity of symptoms, duration of symptoms, or length of time since trauma. We included studies of participants with PTSD as a comorbid disorder, as long as reduction in PTSD symptoms was the primary aim of the intervention. We placed no restriction on the basis or severity of PTSD symptoms or type of traumatic event.

\section{Comorbidities}

We applied no restrictions on the basis of comorbidity.

\section{Setting}

We applied no restrictions on the basis of setting. 


\section{Types of interventions}

\section{Experimental interventions}

We included I-C/BT interventions for the treatment of people with PTSD (with or without therapist guidance), including those delivered online and through native applications. We included programmes based on $\mathrm{CT}, \mathrm{BT}$, or CBT. These terms were defined as follows.

- Interventions based on CT had to incorporate components that aimed to identify and modify unhelpful cognitions.

- Interventions based on BT had to change behaviours associated with unhelpful cognitions or fear conditioning. This might have included exposure-based work.

- Interventions based on CBT must have included a combination of components based on $\mathrm{CT}$ and BT.

We drew a distinction between I-C/BT and online psychoeducation, and did not include online psychoeducation.

To be classified as I-C/BT, programmes had to be delivered via a computer or a mobile device. We included programmes that provided a maximum of five hours of therapist guidance, delivered face-to-face or remotely (e.g. telephone, e-mail, instant messaging). We applied no restrictions based on the number of interactions with a therapist, or the length of the online programme.

We excluded interventions based on EMDR and interventions using mindfulness-based approaches, apart from mindfulness-based I-C/ BT.

\section{Comparator interventions}

- Face-to-face psychological therapy (CBT-based).

- Face-to-face psychological therapy (non-CBT-based), categorised EMDR and other therapies (i.e. supportive therapy, non-directive counselling, psychodynamic therapy, and present-centred therapy) in line with the Cochrane Review of psychological therapies for adults with chronic PTSD (Bisson 2013).

- Wait list, repeated assessment, or usual care.

- Internet psychoeducation.

- Internet psychological therapy (non-CBT).

\section{Types of outcome measures}

We included studies that met the above inclusion criteria, regardless of whether they reported on the following outcomes.

\section{Primary outcomes}

- Severity of PTSD symptoms (measured using a standardised scale, such as the Clinician-Administered PTSD Symptom Scale (CAPS-5; Blake 1995), or the PTSD Checklist (PCL-5; Weathers 2013). When a study reported both a clinician-administered scale and a self-report measure, we used the clinicianadministered measure in the meta-analysis).

- Dropouts (measured by the number of participants still in treatment at the end of the intervention).

\section{Secondary outcomes}

- Diagnosis of PTSD after treatment (number of participants who met diagnostic criteria for PTSD in each arm of the study).

- Severity of depressive symptoms (using a standardised scale, e.g. Beck Depression Inventory (BDI; Beck 1961)).

- Severity of anxiety symptoms (using a standardised scale, e.g. Beck Anxiety Inventory (BAl; Beck 1993)).

- Cost-effectiveness (any measures of cost-effectiveness).

- Adverse events (e.g. symptom worsening (taking into account the measurement error of the instrument), relapses to substance use, hospitalisations, suicide attempts, and work absenteeism).

- Quality of life (any measures of quality of life).

\section{Timing of outcome assessment}

We grouped outcome measures according to length of follow-up as follows.

- Post-treatment.

- Follow-up less than six months' post-treatment.

- Follow-up between six months' and one year' post-treatment.

- Follow-up longer than one year' post-treatment.

Our primary outcome point was immediately post-treatment.

\section{Hierarchy of outcome measures}

We produced hierarchies of standardised measures based on their frequency of use within included studies. When a trial reported data from two or more measures of the same outcome, we only used data from the measure ranked highest.

\section{Search methods for identification of studies}

\section{Cochrane Common Mental Disorders Controlled Trials Register (CCMDCTR)}

The Cochrane Common Mental Disorders Group maintains a specialised register of RCTs, the CCMDCTR. This register contains over 40,000 reference records (reports of RCTs) for anxiety disorders, depression, bipolar disorder, eating disorders, self-harm, and other mental disorders within the scope of this Group. The CCMDCTR is a partially studies-based register with more than $50 \%$ of reference records tagged to about 12,500 individually population, intervention, comparison, outcome (PICO)-coded study records. Reports of trials for inclusion in the register are collated from (weekly) generic searches of Ovid MEDLINE (from 1950), Embase (from 1974), and PsycINFO (from 1967); quarterly searches of the Cochrane Central Register of Controlled Trials (CENTRAL); and review-specific searches of additional databases. Reports of trials are also sourced from international trial registries, drug companies, handsearching of key journals, conference proceedings, and other (non-Cochrane) systematic reviews and meta-analyses. Details of CCMD's core search strategies (used to identify RCTs) can be found on the Group's website, with an example of the core MEDLINE search displayed in Appendix 1.

\section{Electronic searches}

The Cochrane Common Mental Disorders Group's Information Specialist ran initial searches on their specialised register (24 September 2015 and 6 May 2016) using the following search terms. 
1. The CCMDCTR-Studies Register:

Condition = (PTSD or *trauma* or "acute stress" or "stress reaction") AND Intervention $=$ (computer ${ }^{\star}$ or internet or web* or online or selfhelp or self-manage* or self-change)

2. The CCMDCTR-References Register was searched using a more sensitive set of terms to identify additional untagged or uncoded reports of RCTs:

\#1. (PTSD or "trauma" or "combat disorder" or "stress reaction" or "acute stress" or "stress disorder" or "war neurosis"):ab,ti,kw,ky,emt,mh,mc

\#2. (self near3 (care or change or guide* or help or intervention or manag $^{\star}$ or support* or train*)):ab,ti,kw,ky,emt,mh,mc

\#3. (android or app or apps or audio* or blog or iCBT or cCBT or $\mathrm{i}-\mathrm{CBT}$ or C-CBT or CD-ROM or "cell phone" or cellphone or chat or computer* or cyber* or distance* or DVD or eHealth or ehealth or "electronic health*" or e-Portal or ePortal or eTherap* or e-therap* or forum * or gaming or "information technolog" or "instant messag*" or internet* or interapy or ipad or i-pad or iphone or i-phone or ipod or i-pod or web* or WWW or "smart phone" or smartphone or "mobile phone" or e-mail* or email* or mHealth or $\mathrm{m}$-health or mobile or multi-media or multimedia or online* or online or "personal digital assistant" or PDA or SMS or "social medi*" or Facebook or software or telecomm* or telehealth* or telemed or telemonitor ${ }^{\star}$ or telepsych*or teletherap* or "text messag*" or texting or tape or taped or video* or YouTube or podcast or virtual ${ }^{\star}$ or remote):ab,ti,kw,ky,emt, $\mathrm{mh}, \mathrm{mc}$

\#4. (\#1 and (\#2 or \#3))

[Key to CRS field tags: ab:abstract; ti:title; kw:CRG keywords; ky:other keywords; emt:EMTREE headings; mh:MeSH headings; mc:MeSH checkwords]

3. The Information Specialist also ran a complementary search on PILOTS (Published International Literature on Traumatic Stress, US Department of Veterans Affairs), using relevant subject headings and search syntax appropriate to this resource (1990 to 24 November 2014 in the first instance; Appendix 2).

4. The searches were updated (1 March 2018), but as the Group's specialised register was out of date at the time, the information specialist ran searches directly on the following databases (Appendix 3):

- Cochrane Central Register of Controlled Trials (CENTRAL) (Issue 2, 2018);

- Ovid MEDLINE (2016 to 1 March 2018);

- Ovid Embase (2016 to Week 9 2018);

- Ovid PsycINFO (2016 to February week 4 2018);

- ProQuest PILOTS (all years to 2 March 2018);

- CCMDCTR (May to June 2016).

5. We searched international trial registries via the World Health Organization (WHO) International Clinical Trials Registry Platform (ICTRP) and ClinicalTrials.gov to identify unpublished or ongoing studies (to March 2018).

We did not restrict the searches by date, language, or publication status.

\section{Searching other resources}

\section{Grey literature}

We searched sources of grey literature including dissertations and theses, clinical guidelines, and reports from regulatory agencies (when appropriate).

- ProQuest Dissertations and Theses Database.

- National Guideline Clearing House (guideline.gov/).

- Worldwide Regulatory Agencies (www.globepharm.org/links/ resource_agencies.html).

- Open Grey (www.opengrey.eu/).

\section{Reference lists}

We scrutinised the reference lists of all included studies and relevant systematic reviews to identify additional missed studies. We also conducted a cited reference search on the Web of Science.

\section{Correspondence}

We contacted trialists and subject matter experts for information on unpublished or ongoing studies, and to request additional trial data.

\section{Data collection and analysis}

We followed guidance on data collection and analysis provided by the Cochrane Handbook for Systematic Reviews of Interventions (Higgins 2011).

\section{Selection of studies}

Two review authors ( $C L$ and $A B$ ) independently screened titles and abstracts of studies identified by the search, and coded them as 'retrieve' or 'do not retrieve'. We retrieved the full-text publications of all potentially eligible studies, and the same two review authors independently screened and identified studies for inclusion. We recorded reasons for exclusion of ineligible studies. We resolved disagreements through discussion with a third review author (NR), and recorded the selection process in sufficient detail to complete a PRISMA flow diagram and Characteristics of excluded studies table.

\section{Data extraction and management}

We used a data extraction form that was piloted on one study in the review to extract study characteristics and outcome data.

Two review authors ( $C L$ and $A B$ ) independently extracted the following study characteristics and outcome data from included studies.

- Methods: study design, duration of the study, study setting, withdrawals, year of the study.

- Participants: number, mean age, age range, gender, primary trauma, time since trauma, severity of condition, diagnostic criteria, inclusion criteria and exclusion criteria, comorbidity, multiple traumas, trauma during childhood.

- Interventions: intervention, number of hours of guidance, nature of guidance, training and qualifications of guiding therapists, amount of time spent on the programme, comparison, concomitant and excluded interventions, type of device.

- Outcomes: primary and secondary outcomes specified and collected, time points reported. 
- Notes: funding for trial, notable conflicts of interest of trial authors (e.g. if they were involved in development of the intervention).

We noted in the Characteristics of included studies table if outcome data were not reported in a useable way. We resolved disagreements by consensus, or with involvement of a third review author (NR). One review author (CL) transferred data into Review Manager 5 (Review Manager 2014). We double-checked data by comparing data presented in the systematic review with data provided in the study reports. A second review author (AB) spotchecked study characteristics for accuracy against the trial report.

\section{Assessment of risk of bias in included studies}

Two review authors ( $C L$ and $A B$ or $L R$ ) independently assessed the risk of bias for each study, using the criteria outlined in the Cochrane Handbook for Systematic Reviews of Interventions and listed below (Higgins 2011). We resolved conflicts through discussion with a third review author (NR).

- Random sequence generation.

- Allocation concealment.

- Blinding of participants and personnel.

- Blinding of outcome assessment.

- Incomplete outcome data.

- Selective outcome reporting.

- Other bias (including baseline imbalances, early termination of the trial, researcher allegiance).

We judged each potential source of bias as high, low, or unclear, and provided a supporting quotation from the study report, together with a justification for the judgement, in the 'Risk of bias' table. We summarised risk of bias judgements across different studies for each of the domains listed. We considered blinding separately for different key outcomes when necessary. When information on risk of bias related to unpublished data or correspondence with a trialist, we noted this in the 'Risk of bias' table.

When considering treatment effects, we took into account risk of bias for studies that contributed to that outcome.

For cross-over trials, we also took the following into account.

- Suitability of the cross-over design.

- Possibility of carry-over effects.

- Whether only first period data were available.

- Incorrect analysis.

- Comparability of results with those from parallel-group trials.

\section{Measures of treatment effect}

\section{Dichotomous data}

We analysed dichotomous data as risk ratios (RR) to allow comparison across studies. We presented all outcomes with $95 \%$ confidence intervals (Cls).

\section{Continuous data}

We analysed continuous data as mean differences (MDs) or standardised mean differences (SMDs), to allow comparison across studies. We calculated MDs when all studies within a meta-analysis used the same outcome measure, and SMDs when studies used different measures. We entered data presented on a scale with a consistent direction of effect. We presented all outcomes using 95\% $\mathrm{Cls}$, and undertook meta-analyses only when it was meaningful to do so (i.e. when treatments, participants, and the underlying clinical question were sufficiently similar). We planned to describe skewed data reported as medians and interquartile ranges in a narrative, and when multiple trial arms were reported in a single trial, we planned to include only the relevant arms.

Clinical significance was assessed by taking into account the size of a treatment effect, the severity of the condition being treated, and the adverse effects of the treatment.

\section{Unit of analysis issues}

\section{Cluster-randomised trials}

We decided that, when necessary, we would adjust sample sizes, using an estimate of the intracluster or intraclass correlation coefficient (ICC), which describes the similarity of participants within the same cluster. We planned to derive this from the trial if possible, or from another source, such as a similar study, or from a resource providing examples of ICCs, if data were not available in the trial report.

\section{Cross-over trials}

When a study adopted a cross-over design, we planned to only include outcome data from the first randomisation period, to avoid a carry-over effect.

\section{Studies with multiple treatment groups}

We planned to undertake pair-wise meta-analysis with each arm, depending on the nature of the intervention in each arm and its relevance to the review objectives. We aimed to avoid multiple comparisons to limit the risk of false-positive results. If a study included three or more arms that were relevant to the review, we planned to assess the appropriateness of combining data from two arms if therapies were sufficiently similar, or of using data from the arms of the trial that fit most closely with the review objectives. For studies with multiple treatments arms, some of which are relevant to the review, we still listed the treatment arms in the Characteristics of included studies table. Decisions would follow guidance provided by the Cochrane Handbook for Systematic Reviews of Interventions (Higgins 2011).

\section{Dealing with missing data}

We contacted investigators to verify key study characteristics, and to request missing outcome data. We documented all correspondence with trialists, and reported which trialists responded. The protocol described the use of imputation of missing data; however, only published data were presented in the review. Should we find incidents of inadequate reporting of data in future updates of this review, we will attempt to impute missing data from other available information, in line with guidance provided by the Cochrane Handbook for Systematic Reviews of Interventions (Higgins 2011).

\section{Assessment of heterogeneity}

We assessed studies included in each comparison for clinical heterogeneity in terms of variability in experimental and comparator interventions, participants, settings, and outcomes. To further assess heterogeneity, we used both the $\mathrm{I}^{2}$ statistic and the 
$\mathrm{Chi}^{2}$ test of heterogeneity, and visually inspected the forest plots. We used the following scale suggested by the Cochrane Handbook for Systematic Reviews of Interventions as a guide to interpretation of the $\mathrm{I}^{2}$ statistic (Higgins 2011).

- $0 \%$ to $40 \%$ : might not be important.

- $30 \%$ to $60 \%$ : may represent moderate heterogeneity.

- $50 \%$ to $90 \%$ : may represent substantial heterogeneity.

- $75 \%$ to $100 \%$ : shows considerable heterogeneity.

The $\mathrm{I}^{2}$ statistic was interpreted with consideration of the size and direction of effects, as well as the strength of evidence for heterogeneity.

\section{Assessment of reporting biases}

We specified that if sufficient studies were available in a metaanalysis (10 or more), we would prepare funnel plots and examine these for signs of asymmetry. We specified that if asymmetry was identified, we would consider other possible reasons for this.

\section{Data synthesis}

We pooled data from more than one study when appropriate. We performed random-effects meta-analyses as the main analyses as substantial heterogeneity between trials was anticipated. We conducted fixed-effect analyses as sensitivity analyses to informally compare the results. When studies could not be combined, we summarised them in a narrative.

\section{Subgroup analysis and investigation of heterogeneity}

We specified that we would consider the following possible causes of clinical heterogeneity for exploration, if sufficient data allowed.

- Therapist assistance (e.g. Internet-based interventions delivered with guidance, Internet-based interventions delivered without guidance), as this varies substantially between interventions and may impact trial results.

- Type of therapist assistance (e.g. guidance face-to-face, by telephone, by video conference, by e-mail, by instant messaging).

- Participant subgroups (e.g. veterans, female victims of sexual abuse, police officers), as some subgroups are more difficult to treat than others, and may be more or less suited to an online approach to treatment.

- Type of recruitment (e.g. from media adverts only, from healthcare services only), as this may influence motivation and symptom severity of trial participants.

- Type of CBT (e.g. predominantly CT, predominantly BT, CBT), as this may vary, and may include an efficacy outcome.

- Baseline symptom severity (e.g. high versus low baseline mean symptom severity), on the basis that I-C/BT is commonly thought to be better suited to people with milder symptoms.

- Trauma type and context (e.g. war, childhood abuse, motor vehicle accident), on the basis that I-C/BT is commonly thought to be better suited to people with less complex trauma histories.

- Trauma focus (e.g. trauma-focused versus non-trauma-focused $\mathrm{I}-\mathrm{C} / \mathrm{BT}$ ), as findings from the wider PTSD literature support trauma-focused interventions as most effective.

- Type of device (e.g. computer, smartphone), as availability on a smartphone is thought to improve outcomes.
We intended to keep subgroup analyses to a minimum to avoid issues related to multiple testing, and to only conduct these analyses on primary outcome measures.

\section{Sensitivity analysis}

We specified that we would consider sensitivity analysis to explore possible causes of methodological heterogeneity, if sufficient data allowed. We would base analyses on the following criteria.

- Sequence generation.

- Allocation concealment.

- Blinding of outcome assessment.

- Method of diagnosis (e.g. clinician diagnosis, structured interview, screening tool or questionnaire).

We planned to conduct these analyses for primary outcomes by removing studies with high or unknown risk of bias for these domains.

\section{'Summary of findings' tables}

We evaluated the quality of available evidence using the GRADE approach. We generated 'Summary of findings' tables using GRADEpro GDT software, which imports data from Review Manager 5 (GRADEpro GDT; Review Manager 2014). These tables provided outcome-specific information concerning the overall quality of evidence from studies included in the comparison, the magnitude of effect of the interventions examined, and the sum of available data on outcomes considered. We included information on the first seven outcomes of our review: severity of PTSD symptoms posttreatment, dropouts, diagnosis of PTSD after treatment, severity of depressive symptoms, severity of anxiety symptoms, costeffectiveness, and adverse events. We assessed the quality of evidence using five factors.

- Limitations in study design and implementation of available studies.

- Indirectness of evidence.

- Unexplained heterogeneity or inconsistency of results.

- Imprecision of effect estimates.

- Potential publication bias.

For each outcome, we classified the quality of evidence according to the following categories.

- High quality: further research is very unlikely to change our confidence in the estimate of effect.

- Moderate quality: further research is likely to have an important impact on our confidence in the estimate of effect, and may change the estimate.

- Low quality: further research is very likely to have an important impact on our confidence in the estimate of effect, and is likely to change the estimate.

- Very low quality: we are very uncertain about the estimate.

We downgraded the evidence from high quality by one level for serious (or by two for very serious) study limitations (risk of bias), indirectness of evidence, serious inconsistency, imprecision of effect estimates, or potential publication bias. 'Summary of findings' tables included primary outcome measures and posttreatment outcomes. 


\section{RESULTS}

\section{Description of studies}

See Characteristics of included studies table.

\section{Results of the search}

The initial searches identified 669 potentially relevant studies for consideration, plus 99 studies from other sources. After removing duplicates, we were left with 481 reports. We excluded 435 after assessing titles and abstracts. We obtained full-text papers for the remaining 46; after full inspection, we identified four that met the inclusion criteria and were included in the final qualitative and quantitative analyses (Ivarsson 2014; Lewis 2017; Litz 2007; Spence 2011),
An update search (1 March 2018) identified a further 861 records and after the Information Specialist removed 359 duplicate records and 161 reports of uncontrolled trials, left 341 records to screen. After we assessed the abstracts and relevant full-text, we identified four additional studies that met inclusion criteria (Krupnick 2017; Kuhn 2017; Littleton 2016; Miner 2016). These have been included in the final qualitative and quantitative analyses together with two studies which were previously awaiting classification (Engel 2015; Knaevelsrud 2015).

We excluded 38 studies (see Characteristics of excluded studies table).

We identified 13 ongoing studies (see Characteristics of ongoing studies table).

The process of study selection is illustrated in the PRISMA diagram in Figure 1. 
Figure 1. Study flow diagram.

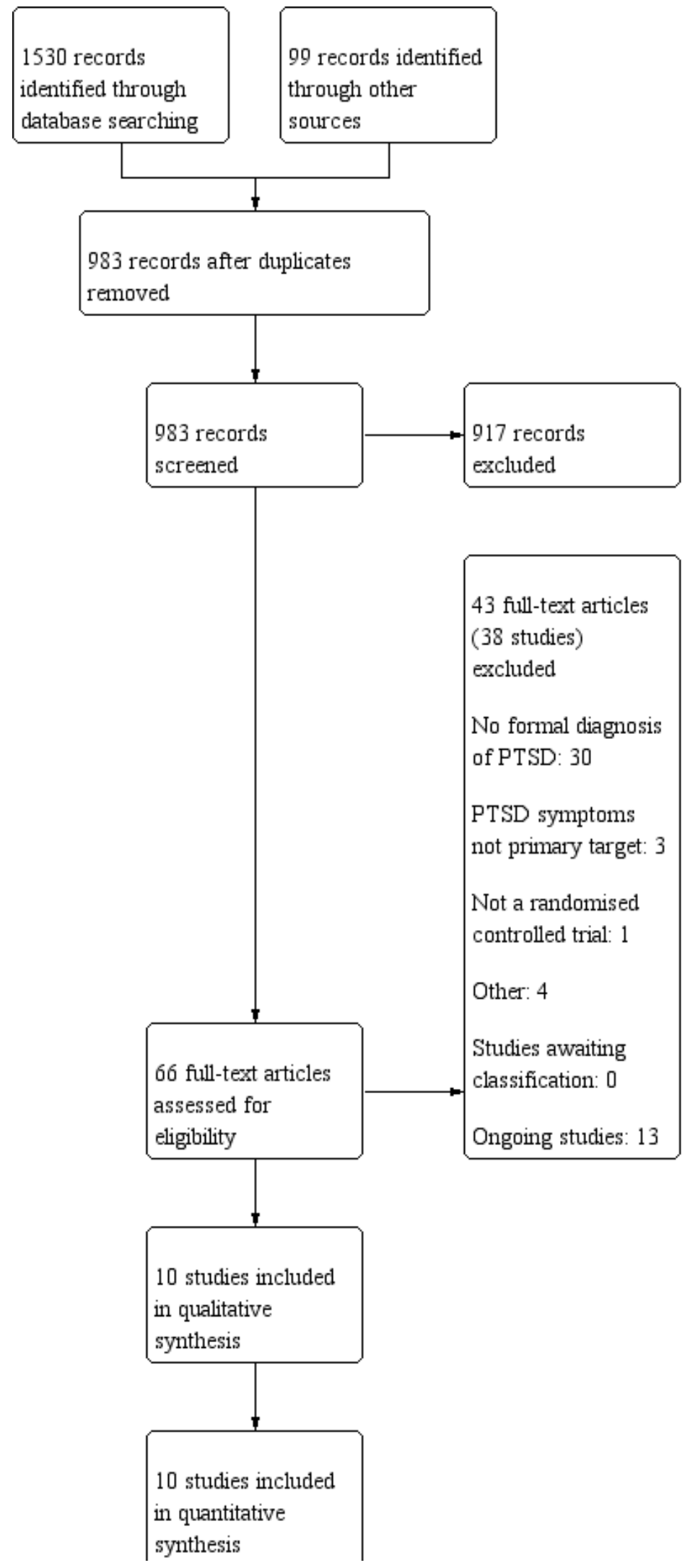


Figure 1. (Continued)

$$
\begin{aligned}
& \text { ㅍ quanulauve } \\
& \text { synthesis } \\
& \text { (meta-analysis) }
\end{aligned}
$$

\section{Included studies}

The review included 10 RCTs of 720 participants.

\section{Design}

All of the included studies were RCTs. All studies randomly assigned participants, as opposed to clinics or practices.

\section{Sample sizes}

The studies had sample sizes of 80 (I-C/BT 43; optimised usual care 37; Engel 2015); 62 (I-C/BT 31; delayed treatment 31; Ivarsson 2014); 159 (I-C/BT 79; wait list 80; Knaevelsrud 2015); 31 (I-C/BT 16; treatment as usual 15; Krupnick 2017); 120 (I-C/BT 62; wait list 58; Kuhn 2017); 42 (I-C/BT 21; wait list 21; Lewis 2017); 87 (I-C/BT 46; supportive counselling 41; Littleton 2016); 45 (I-C/BT 24; supportive counselling 21; Litz 2007); 49 (I-C/BT 25; wait list 24; Miner 2016), and 42 (I-C/BT 21; wait list 21; Spence 2011).

\section{Setting}

Six studies were conducted in the US (Engel 2015; Krupnick 2017; Kuhn 2017; Littleton 2016; Litz 2007; Miner 2016), one in Sweden (Ivarsson 2014), one in Australia (Spence 2011), one in the UK (Lewis 2017), and one in Iraq (Knaevelsrud 2015).

\section{Participants}

One included participants who met criteria for DSM-5 PTSD (Lewis 2017); the other nine studies included participants who met criteria for Diagnostic and Statistical Manual 4th Edition (DSM-IV) PTSD (Engel 2015; Ivarsson 2014; Knaevelsrud 2015; Krupnick 2017; Kuhn 2017; Littleton 2016; Litz 2007; Miner 2016; Spence 2011). One study included only military personnel traumatised after combat exposure in Afghanistan or Iraq, or the 9/11 attacks on the Pentagon (Litz 2007); one study included only female rape victims (Littleton 2016), and the remainder included participants traumatised after a variety of traumatic events that met DSM criteria. See Characteristics of included studies table for further details. Where reported, the percentage of women in studies ranged from $18.75 \%$ to $100 \%$; the percentage of participants with a university education ranged from $14.2 \%$ to $62.8 \%$; and the percentage of participants who were unemployed ranged from $8.1 \%$ to $40 \%$.

\section{Interventions}

Eight studies compared an Internet programme based on traumafocused CBT with a wait list, treatment as usual, or delayed treatment control group (Engel 2015; Ivarsson 2014; Knaevelsrud 2015; Krupnick 2017; Kuhn 2017; Lewis 2017; Miner 2016; Spence 2011). Treatment durations were four weeks (Miner 2016), five weeks (Knaevelsrud 2015), six weeks (Engel 2015), eight weeks (Ivarsson 2014; Lewis 2017; Spence 2011), 10 sessions (Krupnick 2017) and 12 weeks (Kuhn 2017).

Two studies compared an Internet programme based on traumafocused CBT with Internet psychological therapy (non-CBT) (Littleton 2016; Litz 2007). In the Litz 2007 study, the duration of treatment was eight weeks, while in the study by Littleton 2016, treatment was for 14 weeks. A therapist guided all of the Internet interventions. See Characteristics of included studies table for further details.

\section{Outcomes}

Symptoms of PTSD were measured using the Impact of Event Scale (IES-R), the Clinician-Administered PTSD Scale (CAPS-5), the PTSD Checklist - Civilian Version (PCL-C), the PTSD Symptom Scale Interview (PSS-I), and the Posttraumatic Diagnostic Scale (PDS). Depression was measured using the Beck Depression Inventory (BDI), the Patient Health Questionnaire (PHQ-7, PHQ-8, PHQ-9, PHQ-15), and the Center for Epidemiologic Studies Depression Scale (CES-D). Anxiety was measured using the Beck Anxiety Inventory (BAI) and the Generalized Anxiety Disorder 7-Item Scale (GAD-7). Quality of life was measured using the Quality of Life Inventory (QOLI) and EUROHIS-QUOL.

\section{Excluded studies}

For details of the 38 excluded studies, see the Characteristics of excluded studies table.

\section{Ongoing studies}

For details of the 13 ongoing studies, see the Characteristics of ongoing studies table.

\section{Studies awaiting classification}

There are no studies awaiting classification.

\section{Risk of bias in included studies}

For details of the risk of bias judgements for each study, see the Characteristics of included studies table. A graphical representation of the overall risk of bias in included studies is presented in Figure 2 and Figure 3. 
Figure 2. 'Risk of bias' graph: review authors' judgements about each risk of bias domain presented as percentages across all included studies.

Random sequence generation (selection bias)

Allocation concealment (selection bias)

Blinding of participants and personnel (performance bias): All outcomes

Blinding of outcome assessment (detection bias): All outcomes Incomplete outcome data (attrition bias): All outcomes

Selective reporting (reporting bias)

Other bias
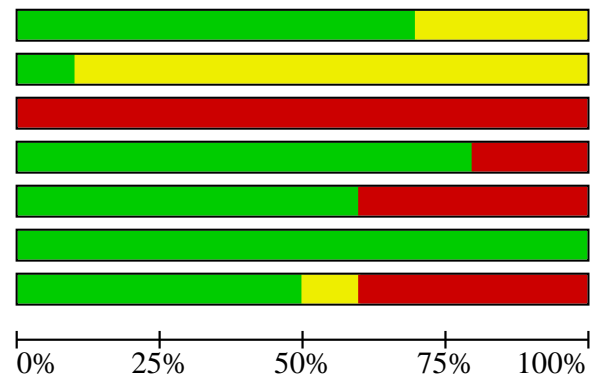
Figure 3. 'Risk of bias' summary: review authors' judgements about each risk of bias domain for each included study.

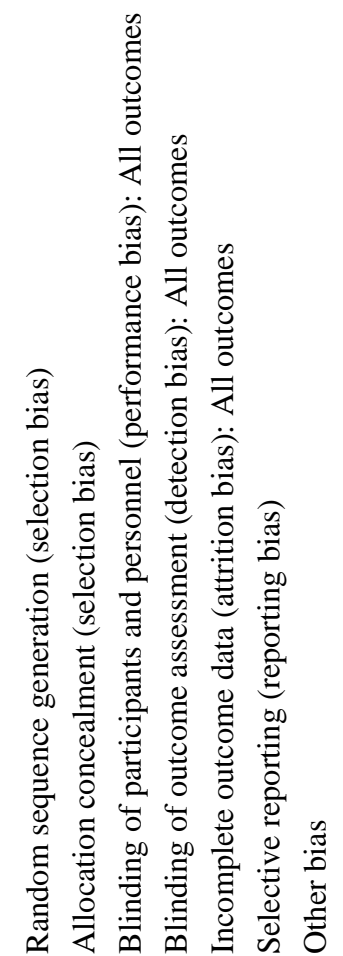

Engel 2015

Ivarsson 2014

Knaevelsrud 2015

Krupnick 2017

Kuhn 2017

Lewis 2017

Littleton 2016

Litz 2007

Miner 2016

Spence 2011

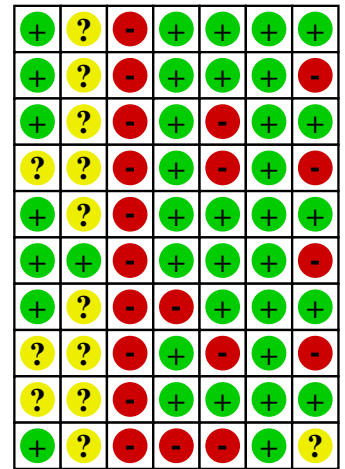

For cluster-randomised trials, we planned to take the following into account.

- Recruitment bias (e.g. if participants had been recruited to the trial after clusters were randomly assigned).

- Baseline imbalance.

- Loss of clusters.

- Incorrect analysis.

- Comparability with individual randomised trial

\section{Allocation}

Seven of the included studies provided sufficient information to determine that there was a low risk of bias associated with sequence generation (Engel 2015; Ivarsson 2014; Knaevelsrud 2015;
Kuhn 2017; Lewis 2017; Littleton 2016; Spence 2011), while three studies did not provide information to make a judgement (Krupnick 2017; Litz 2007; Miner 2016). One study reported the use of sealed, opaque envelopes to conceal the allocation of treatment and was judged at low risk of selection bias (Lewis 2017). The remaining nine studies did not provide information to make a judgement on allocation concealment and were therefore classified at unclear risk of selection bias (Engel 2015; Ivarsson 2014; Knaevelsrud 2015; Krupnick 2017; Kuhn 2017; Littleton 2016; Litz 2007; Spence 2011).

\section{Blinding}

It is impossible to blind the participants and therapists in psychological treatment trials and, therefore, all 10 studies were at high risk of performance bias. 
Eight studies reported adequate blinding of the outcome assessor (Engel 2015; Ivarsson 2014; Knaevelsrud 2015; Krupnick 2017; Kuhn 2017; Lewis 2017; Litz 2007; Miner 2016). The two remaining studies reported that outcome assessors were not blinded to treatment and were, therefore, at high risk of detection bias (Littleton 2016; Spence 2011).

\section{Incomplete outcome data}

Six of the included studies dealt with missing outcome data appropriately and were judged to be at low risk of attrition bias (Engel 2015; Ivarsson 2014; Kuhn 2017; Lewis 2017; Littleton 2016; Miner 2016). Four studies were at high risk of attrition bias (Knaevelsrud 2015; Krupnick 2017; Litz 2007; Spence 2011). One study completed intention-to-treat (ITT) analysis but missing data were more than 30\% (Knaevelsrud 2015). One study had a dropout rate of over $75 \%$ and did not fully report reasons for dropout (Krupnick 2017). One study reported completer data only, but performed ITT analyses (Litz 2007). One study used the last outcome carried forward method to impute missing data (Spence 2011). Reasons for dropout were poorly described.

\section{Selective reporting}

Only one of the included studies published the study protocol (Knaevelsrud 2015). Of the nine remaining included studies, although the study protocol was not available, it was clear that the published reports included the outcomes PTSD, depression, and anxiety, that were prespecified and expected in trials of this type in the field of PTSD. Therefore, these nine studies were judged at low risk of reporting bias (Engel 2015; Ivarsson 2014; Krupnick 2017; Kuhn 2017; Lewis 2017; Littleton 2016; Litz 2007; Miner 2016; Spence 2011).

\section{Other potential sources of bias}

Five studies were at low risk of other bias (Engel 2015; Knaevelsrud 2015; Kuhn 2017; Littleton 2016; Miner 2016). Four studies were at high risk of bias (Ivarsson 2014; Krupnick 2017; Lewis 2017; Litz 2007). We could not rule out potential researcher allegiance, since three included trials were of interventions that were evaluated by their originators (Ivarsson 2014; Lewis 2017; Litz 2007). Sample sizes in three studies were small (Krupnick 2017; Lewis 2017; Litz 2007), and follow-up was very limited in one study (Litz 2007). For practical and ethical reasons, longer-term follow-up data were not available from the wait list groups. One study was at unclear risk of bias as it terminated recruitment prematurely, and failed to recruit the prespecified number of participants (Spence 2011).

\section{Effects of interventions}

See: Summary of findings 1 Internet-based cognitive and behavioural therapy (I-C/BT) compared to wait list for posttraumatic stress disorder (PTSD) in adults; Summary of findings 2 Internet-based cognitive and behavioural therapy (I-C/BT) compared to I-non-C/BT for post-traumatic stress disorder (PTSD) in adults

\section{Comparison 1: Internet-based cognitive and behavioural therapies versus face-to-face cognitive behavioural therapy}

None of the included studies compared I-C/BT versus face-to-face CBT.
Comparison 2: Internet-based cognitive and behavioural therapies versus face-to-face non-cognitive behavioural therapy

None of the included studies compared I-C/BT versus face-to-face non-CBT.

\section{Comparison 3: Internet-based cognitive and behavioural therapies versus wait list or usual care}

Eight studies including 560 participants compared I-C/BT versus wait list or usual care (Engel 2015; Ivarsson 2014; Knaevelsrud 2015; Krupnick 2017; Kuhn 2017; Lewis 2017; Miner 2016; Spence 2011). See Summary of findings 1.

\section{Primary outcomes}

\subsection{Severity of post-traumatic stress disorder symptoms}

There was very low-quality evidence that I-C/BT was more effective than wait list when the severity of PTSD symptoms were measured post-treatment (SMD $-0.60,95 \% \mathrm{Cl}-0.97$ to -0.24 ; participants $=560$; studies $=8$; Analysis 1.1). There was considerable heterogeneity in study results $\left(1^{2}=76 \%\right)$. When duration of followup was less than six months, there was no evidence of a difference between I-C/BT and wait list groups (SMD $-0.43,95 \% \mathrm{Cl}-1.41$ to 0.56 ; studies $=3$; participants = 146; Analysis 1.2).

\subsection{Dropouts}

There was low-quality evidence of a significant difference in dropout rates from the I-C/BT and wait list groups (RR 1.39, 95\% Cl 1.03 to 1.88 ; studies $=8$; participants $=585 ; I^{2}=13 \%$; Analysis 1.3 ).

\section{Secondary outcomes}

\subsection{Diagnosis of post-traumatic stress disorder after treatment}

There was very low-quality evidence that I-C/BT was no more effective than wait list at reducing the risk of a diagnosis of PTSD after treatment (RR $0.53,95 \% \mathrm{Cl} 0.28$ to 1.00 ; participants $=62$; studies $=1$; Analysis 1.4).

\subsection{Severity of depressive symptoms}

There was very low-quality evidence that I-C/BT was more effective than wait list at reducing the severity of depressive symptoms (SMD $-0.61,95 \% \mathrm{Cl}-1.17$ to -0.05 ; participants $=425$; studies $=5$; Analysis 1.5). There was considerable heterogeneity in study results $\left(\mathrm{I}^{2}=\right.$ $86 \%)$. There was very low-quality evidence that I-C/BT was still more effective than wait list at follow-up less than six months (MD $8.95,95 \% \mathrm{Cl}-15.57$ to -2.33 ; participants $=42$; studies $=1$; Analysis 1.6).

\subsection{Severity of anxiety symptoms}

There was very low-quality evidence that I-C/BT was more effective than wait list at reducing symptoms of anxiety (SMD $-0.67,95 \% \mathrm{Cl}$ -0.98 to -0.36 ; participants $=305$; studies $=4 ; I^{2}=35 \%$; Analysis 1.7). There was very low-quality evidence that I-C/BT was still more effective than wait list at follow-up less than six months (MD -12.59, $95 \% \mathrm{Cl}-20.74$ to -4.44 ; participants $=42$; studies $=1$; Analysis 1.8$)$.

\subsection{Cost-effectiveness}

None of the included studies comparing I-C/BT to wait list or usual care reported cost-effectiveness. 


\subsection{Adverse events}

None of the included studies comparing I-C/BT to wait list or usual care reported adverse events.

\subsection{Quality of life}

There was very low-quality evidence of no significant difference between the I-C/BT and wait list groups for quality of life (SMD 0.60, $95 \% \mathrm{Cl} 0.08$ to 1.12; participants $=221$; studies $=2$; Analysis 1.9) . There was substantial heterogeneity in study results $\left(I^{2}=68 \%\right)$.

\section{Comparison 4: Internet-based cognitive and behavioural therapies versus Internet psychoeducation}

None of the included studies compared I-C/BT versus Internet psychoeducation.

\section{Comparison 5: Internet-based cognitive and behavioural therapies versus Internet-based non-cognitive and behavioural therapies}

Two studies including 82 participants compared I-C/BT versus Internet-based non-cognitive and behavioural therapies (I-non-C/ BT) (Littleton 2016; Litz 2007). See Summary of findings 2.

\subsection{Severity of post-traumatic stress disorder symptoms}

There was very low-quality evidence of no significant difference between the I-C/BT and I-non-C/BT groups in the post-treatment severity of PTSD symptoms (SMD $-0.08,95 \% \mathrm{Cl}-0.52$ to 0.35 ; participants $=82$; studies $=2 ;\left.\right|^{2}=19 \%$; Analysis 2.1 ), or at follow-up less than six months (SMD $0.08,95 \% \mathrm{Cl}-0.41$ to 0.57 ; participants $=65$; studies = 2; $\left.\right|^{2}=0 \%$; Analysis 2.2). However, there was a significant difference in favour of I-C/BT at follow-up of six to 12 months (MD $-8.83,95 \% \mathrm{Cl}-17.32$ to -0.34 ; participants $=18$; studies $=1$; Analysis 2.3 ).

\subsection{Dropouts}

There was very low-quality evidence of no significant difference between dropout rates from the I-C/BT and I-non-C/BT (RR 2.14, $95 \% \mathrm{Cl} 0.97$ to 4.73 ; participants $=132 ;$ studies $=2 ; I^{2}=0 \%$; Analysis 2.4).

\section{Secondary outcomes}

\subsection{Diagnosis of post-traumatic stress disorder after treatment}

Neither study comparing I-C/BT versus I-non-C/BT reported on diagnosis of PTSD after treatment.

\subsection{Severity of depressive symptoms}

There was very low-quality evidence of no significant difference in severity of depressive symptoms between the I-C/BT and I-nonC/BT groups post treatment (SMD $-0.12,95 \% \mathrm{Cl}-0.78$ to 0.54 ; participants $=84 ;$ studies $=2 ; I^{2}=52 \%$; Analysis 2.5$)$ or when follow-up was less than six months (SMD $0.20,95 \% \mathrm{Cl}-0.31$ to $0.71 ;$ participants $=61 ;$ studies $=2 ; I^{2}=0 \%$; Analysis 2.6). However, there was very low-quality evidence of a difference in severity of depressive symptoms between the I-C/BT and I-non-C/BT groups when follow-up was six to 12 months (MD $-8.34,95 \% \mathrm{Cl}-15.83$ to 0.85 ; participants $=18$; studies $=1$; Analysis 2.7 ).

\subsection{Severity of anxiety symptoms}

There was very low-quality evidence of no significant difference in severity of symptoms of anxiety between the I-C/BT and I-non-C/BT groups (SMD 0.08, 95\% Cl -0.78 to 0.95 ; participants $=74$; studies = $2 ; 1^{2}=70 \%$; Analysis 2.8) or when follow-up was less than six months (SMD $-0.16,95 \% \mathrm{Cl}-0.67$ to 0.35 ; participants $=60$; studies $=2 ; \mathrm{I}^{2}=$ $9 \%$; Analysis 2.9). However, there was very low-quality evidence of a difference in severity of anxiety symptoms between the I-C/BT and I-non-C/BT groups when follow-up was six to 12 months (MD -8.05, $95 \% \mathrm{Cl}-15.20$ to -0.90 ; participants $=18$; studies $=1$; Analysis 2.10).

\subsection{Cost-effectiveness}

Neither study comparing I-C/BT versus I-non-C/BT reported costeffectiveness.

\subsection{Adverse events}

Neither study comparing I-C/BT versus I-non-C/BT reported adverse effects.

\subsection{Quality of life}

Neither study comparing I-C/BT versus I-non-C/BT reported quality of life.

\section{Subgroup analyses}

There were insufficient data to perform subgroup analyses on the effect of therapist assistance; type of therapist assistance; participant subgroups; type of recruitment; type of CBT; baseline symptom severity; trauma type and context; trauma focus; or type of device.

\section{Sensitivity analyses}

We could not conduct sensitivity analyses because none of the comparisons included more than 10 studies.

\section{DISCUSSION}

\section{Summary of main results}

The review included 10 studies with 720 participants (see Summary of findings 1; Summary of findings 2). Eight of the studies compared I-C/BT delivered with therapist guidance to a wait-list control group. Two studies compared guided I-C/BT with I-non-C/BT.

Therapist-guided I-C/BT may have been more effective than a wait list in reducing PTSD symptoms, depression, and anxiety posttreatment. However, there was no significant difference between the two groups on measures of quality of life. The magnitude of effect was smaller than that found in comparisons of therapistadministered trauma-focused CBT with wait list or usual care (Bisson 2013). This may indicate that while beneficial, this is a less effective form of treatment. There was a significant difference in dropout rates between groups, with a greater number leaving I-C/ BT than wait list/usual care.

There was no significant difference between therapist-guided I-C/ BT and I-non-C/BT on any measure post-treatment. Only two small studies made this comparison; they showed that I-C/BT was no more effective in reducing severity of PTSD symptoms, anxiety, and depression at 12-month follow-up. There was no significant difference in dropout rates between groups.

\section{Overall completeness and applicability of evidence}

The field of I-C/BT for PTSD is in its infancy, and this review considered the limited number of studies currently available. 
Drawing together the results of these studies provided an indication of the effectiveness of I-C/BT. However, the small number of studies contributing to each comparison limited our ability to comprehensively answer the questions that we set out to address. Indeed, we were unable to answer many of the questions set out by the review. For example, we were unable to draw any conclusions related to cost-effectiveness. We were also unable to compare the efficacy of I-C/BT with face-to-face psychological therapy or the provision of education. This indicates a need for further research, and an update of the review once further studies have been completed.

Eligible RCTs included adults who had been exposed to a variety of traumatic events (see Types of participants). We included all studies in which at least $70 \%$ of participants had been diagnosed with PTSD. We excluded studies that evaluated I-C/BT in participants with subthreshold PTSD symptoms, or traumatised people who were not formally diagnosed as having PTSD (see Characteristics of excluded studies table). This approach is in keeping with the Cochrane Review of therapist-administered psychological therapies for PTSD, and the aim was to ensure the empirical validity of the review (Bisson 2013). However, this did result in the exclusion of studies that would have contributed additional data.

The included studies were conducted in Sweden, the UK, the USA, Iraq, and Australia, which limits generalisability of results to the rest of the world, especially to low- and middle-income countries. The studies did not include participants with comorbidities of substance dependence, psychosis, and severe depression, therefore, excluding people who are arguably more difficult to treat. That said, it is possible that I-C/BT may be most appropriate for people with mild-to-moderate PTSD, in a stepped or stratified pathway of care. Participants included in the 10 studies were predominantly white, employed, and had relatively high levels of education. Therefore, it is not possible to determine whether similar results would have been obtained from participants with more representative demographic characteristics.

Eight of the 10 included studies compared I-C/BT to a wait list or delayed treatment control group. The search identified no studies that compared I-C/BT to psychological therapy delivered face-to-face (CBT-based or non-CBT-based), or to Internet-based psychoeducation. Therefore, we were unable to draw any conclusions related to these comparisons. All studies included some level of guidance, which precluded comparison of guided and unguided interventions. None of the eligible studies included data on cost effectiveness, so we were unable to comment on whether these interventions hold promise from an economic standpoint, although clearly, they would have cost less to deliver than faceto-face therapy, given the reduced contact time with therapists. Despite high levels of dropout from the included studies, the reasons given for this were poorly described, which precluded indepth consideration related to the tolerability of interventions.

As is common in studies of psychological therapies, concurrent pharmacotherapy was permissible. This caused issues disentangling the effects of medication versus the therapy being trialled. This is largely unavoidable, due to ethical considerations, and all studies stipulated that dosage had been constant for a stipulated duration. Although concurrent psychological therapy was an exclusion criterion for the included studies, methods of formally evaluating whether additional treatment had been sought were not described. Indeed, one study gave the possibility of participants in the control group having engaged in psychological therapy during the study period as a possible explanation for a failure to find between group differences, despite large withingroup differences in the treatment group (Spence 2011).

To ensure consistency with other Cochrane Reviews of psychological therapies for PTSD, there was a requirement that at least $70 \%$ of participants met diagnostic criteria for PTSD. This resulted in us excluding several studies of traumatised people with subthreshold PTSD symptoms from the review. It may be argued that this limits the clinical relevance of the review, since Internetbased interventions may be useful to people with subthreshold PTSD symptoms, and their use not limited to those meeting full diagnostic criteria. However, it is intuitive that interventions that are effective for people meeting the criteria for a diagnosis will also be effective in reducing traumatic stress symptoms among people with subthreshold symptomology. Therefore, restricting the review to studies with clinical samples takes a conservative approach, and, consistent with the aims of the review, provides an indication of whether I-C/BT is effective for the treatment of clinically significant PTSD symptoms. It is also worth noting that people who have volunteered to be part of a trial may engage more with I-C/BT than the general population of people with PTSD, which may have an impact on results.

There were insufficient data to perform subgroup analyses and determine the potential influence of therapist assistance; type of therapist assistance; participant subgroups; type of recruitment; type of CBT; baseline symptom severity; trauma type and context; trauma-focus; or type of device.

\section{Quality of the evidence}

We used the five GRADE considerations (study limitations, consistency of effect, imprecision, indirectness, and publication bias) to assess the quality of the body of evidence for each outcome, and to draw conclusions about the quality of evidence within the text of the review. One of the main considerations for downgrading GRADE judgements was risk of bias. Concerns over the quality of the evidence also limit the extent to which conclusions can be generalised. For details of the risk of bias judgements for each study, see the Characteristics of included studies table and the graphical representation of risk of bias presented in Figure 2 and Figure 3.

There was heterogeneity across the I-C/BT programmes, which varied in content, delivery, and guidance. Although all were based on cognitive-behavioural principles, the exact nature of what was included varied. The extent and method by which the Internet-based therapies were guided by a trained professional also varied. These interventions were compared to wait list or delayed treatment control groups (eight studies) or Internet-based noncognitive/behavioural therapy (two studies).

\section{Potential biases in the review process}

The review rigorously followed guidelines set out by Cochrane (Higgins 2011). Two review authors independently screened the abstracts identified by the literature search; read all potentially relevant studies; assessed each study against the inclusion criteria; extracted data from the written reports; and rated each study for risk of bias. We discussed any disagreements with a third review author, and reached unanimous decisions for inclusion and 
classification. We carefully followed guidelines set out by Cochrane on statistical methods and used GRADE to assess the quality of evidence (Higgins 2011). Following these procedures minimised the potential for bias, but some unavoidable issues remained.

All studies included in the review were published, which led to the possibility of publication bias. Since the meta-analyses included fewer than 10 studies, we were unable to use funnel plots to visually explore this possibility (Higgins 2011). In an attempt to overcome this limitation, we searched clinical trial registries with the aim of identifying unpublished trials. That said, we could not rule out the possibility that trials were not registered, which may be the case for smaller studies, pilot work, or unfunded research.

We systematically searched numerous online databases for potentially relevant studies, and scrutinised reference lists of included studies. We also contacted experts in the field requesting help to identify missed studies or ongoing work. Nonetheless, we could not fully eliminate the possibility of overlooked RCTs. The included studies appeared to report data for all outcomes of primary interest. However, we could not identify published protocols for the included studies, and, therefore, it was possible that other outcomes of interest were collected but not reported.

There was considerable statistical heterogeneity in two of the four pooled comparisons. In circumstances where heterogeneity was thought to be potentially problematic, we used a randomeffects model. Heterogeneity was also a factor that was taken into consideration in downgrading the quality of the evidence with GRADE.

\section{Agreements and disagreements with other studies or reviews}

One Cochrane Review found that trauma-focused I-C/BT interventions showed a smaller effect than therapist-administered interventions in comparison to wait list (Bisson 2013). In part, this may have been due to the small number of studies and the fact that the study by Spence 2011 found a high level of symptom improvement in the control group. Our review also differed from the review by Bisson 2013 because we found no significant difference between trauma-focused CBT and non-CBT based psychological therapy, although this may be a function of limited power.

The comparison of I-C/BT to wait list in the current review yielded a larger effect size than the equivalent comparison in a previous Cochrane Review of media-delivered CBT for anxiety disorders in adults (Mayo-Wilson 2013). The effect size was of a similar magnitude to that obtained in the equivalent comparison in a Cochrane Review of I-C/BT for anxiety disorders, which excluded interventions provided without therapist assistance, and interventions that included face-to-face therapist assistance (Olthuis 2015). However, these comparisons should be treated with caution, due to the small number of studies and high level of heterogeneity in the studies included in the current review.

\section{AUTHORS' CONCLUSIONS}

\section{Implications for practice}

Internet-based cognitive and behavioural therapy (I-C/BT) has grown in popularity due to its potential to broaden access to cost-effective treatment in a climate of limited resources, ever increasing pressure on psychological services, and public preference to use the Internet. The interventions considered by this review required significantly less therapist time than current firstline treatments, creating an opportunity to increase therapeutic capacity and optimise access to evidence-based treatment. It had several potential benefits, including reduced distress and improved functioning. While the review found some beneficial effects of I-C/ BT for PTSD, the quality of the evidence was very low due to the small number of studies included trials.

\section{Implications for research}

The field of I-C/BT for post-traumatic stress disorder (PTSD) has lagged behind other disorders, and only a handful of studies have evaluated the efficacy of the approach within a clinical population. Replication of findings is thereby a continued requirement. There have been no randomised controlled trials to date comparing I-C/BT for PTSD with evidence-based therapistadministered psychological therapy. None of the included studies reported on adverse events.

Although systematic reviews of self-help interventions for other disorders have reported better outcomes for guided interventions compared to those without guidance, this has not been explored within the field of PTSD. There have been no trials comparing Internet cognitive behavioural therapy (CBT) with or without guidance, and no attempts to ascertain the optimal level of therapist input. In addition, we currently have a poor understanding of who can benefit from Internet-CBT for PTSD. All studies were conducted in high-income countries and participants in the included trials were predominantly white and highly educated. It is unknown how results would generalise to other populations. There is potential for I-C/BT to have an important role in the provision of global mental healthcare. It could be especially useful in low- and middle-income countries, where fewer appropriately trained clinicians are available. However, further work is needed to explore the feasibility of using I-C/BT in these countries, and to determine the acceptability of the approach within other countries.

Dropout from Internet-based therapies is a concern, and our current understanding of predictors of dropout is inadequate, indicating the need to further explore this within future trials. There is a need to explore predictors of outcome and dropout, such as participant age, trauma type, levels of computer literacy, and symptom severity. This will provide a greater understanding of the best candidates for Internet-based CBT, and enable interventions to be targeted accordingly. Future studies exploring the feasibility of I-C/BT and willingness to engage with Internet-based therapies would be valuable. We know little in terms of the long-term effects of $\mathrm{I}-\mathrm{C} / \mathrm{BT}$, indicating a need for future trials to include longerterm follow-up. Further work is also needed to back-up a common assumption that I-C/BT is cost effective, and to evaluate this for the treatment of PTSD.

We were unable to draw any conclusions related to cost effectiveness. We were also unable to compare the efficacy of IC/BT with face-to-face psychological therapy or the provision of psychoeducation. This indicates a need for further research, and an update of the review once further studies have been completed. 


\section{ACK N O WLEDGEMENTS}

With thanks to the editorial team of the Cochrane Common Mental Disorder Group.

CRG Funding Acknowledgement
The National Institute for Health Research (NIHR) is the largest single funder of the Cochrane Common Mental Disorders Group.

\section{Disclaimer}

The views and opinions expressed herein are those of the review authors and do not necessarily reflect those of Cardiff University, the NIHR, the National Health Service, or the Department of Health and Social Care. 


\section{R E F E R E N C E S}

\section{References to studies included in this review}

Engel 2015 \{published data only\}

Engel CC, Litz B, Magruder KM, Harper E, Gore K, Stein N, et al. Delivery of self training and education for stressful situations (DESTRESS-PC): a randomized trial of nurse assisted online self-management for PTSD in primary care. General Hospital Psychiatry 2015; 37(4):323-8. [NCT01474057]

Ivarsson 2014 \{published data only\}

Ivarsson D, Blom M, Hesser H, Carlbring P, Enderby P, Nordberg R, et al. Guided Internet-delivered cognitive behavior therapy for post-traumatic stress disorder: a randomized controlled trial. Internet Interventions 2014; 1:35-40.

\section{Knaevelsrud 2015 \{published data only\}12611001019998}

Knaevelsrud C, Brand J, Lange A, Ruwaard J, Wagner B. Webbased psychotherapy for posttraumatic stress disorder in wartraumatized Arab patients: randomized controlled trial. Journal of Medical Internet Research 2015; 17(3):e71.

\section{Krupnick 2017 \{published data only\}}

Krupnick JL, Green BL, Amdur R, Alaoui A, Belouali A, Roberge E, et al. An Internet-based writing intervention for PTSD in veterans: a feasibility and pilot effectiveness trial. Psychological Trauma: Theory, Research, Practice and Policy 2017; 9(4):461-70.

\section{Kuhn 2017 \{published data only\}}

Kuhn E, Kanuri N, Hoffman JE, Garvet DW, Ruzek JI. A randomized controlled trial of a smartphone app for posttraumatic stress disorder symptoms. Journal of Consulting and Clinical Psychology 2017; 85(3):267-73.

. PTSD coach app evaluation. ClinicalTrials.gov/show/ NCT02445196 Date first received: 15 May 2015

\section{Lewis 2017 \{published data only\}}

Lewis C, Farewell D, Groves V, Kitchiner N, Roberts N, Vick T, et al. Internet-based guided self-help for post-traumatic stress disorder (PTSD): randomised controlled trial. Depression and Anxiety 2017; 34(6):555-65.

\section{Littleton 2016 \{published data only\}}

Littleton H, Grills AE, Kline KD, Schoemann AM, Dodd JC. The From Survivor to Thriver program: RCT of an online therapistfacilitated program for rape-related PTSD. Journal of Anxiety Disorders 2016; 43:41-51.

. Evaluation of web-based CBT for rape victims ClinicalTrials.gov/show/NCT02777294 Date first received: 19 May 2016

\section{Litz 2007 \{published data only\}}

Litz B, Engel CC, Bryant R, Papa A. A randomized, controlled proof of concept trial of an Internet-based, therapist-assisted self-management treatment for posttraumatic stress disorder. American Journal of Psychiatry 2007; 164:1676-83.
Miner 2016 \{published data only\}

Miner A, Kuhn E, Hoffman JE, Owens JE, Ruzik JI. Feasibility, acceptability, and potential efficacy of the PTSD Coach App: a pilot randomized controlled trial with community trauma survivors. Psychological Trauma: Theory, Research, Practice and Policy 2016; 8(3):384-92.

\section{Spence 2011 \{published data only\}12611000989943}

Spence J, Titov N, Dear BF, Johnston L, Solley K, Lorian C. Randomized controlled trial of Internet-delivered cognitive behavioral therapy for post-traumatic stress disorder. Depression and Anxiety 2011; 28(7):541-50.

\section{References to studies excluded from this review}

Bahena 2016 \{published data only\}

Efficacy of a mobile application among a sample of veterans with symptoms of post-traumatic stress disorder. Dissertation Abstracts International 2016; 77:2.

Bishop 2012 \{published data only\}

Bishop TM, Possemato K, Acosta M, Lantinga LJ, Maisto SA, Marsch L, et al. Moving forward: update on the development of a web-based cognitive behavioral treatment for OEF/ OIF veterans with PTSD symptoms and substance misuse. Alcoholism, Clinical and Experimental Research 2012; 36:347A

Blankenship 2013 \{published data only\}

Blankenship AE. A prospective examination of mindfulness training on the mitigation of postraumatic stress symptoms thesis, 2013. scholarworks.wmich.edu/dissertations/161 (accessed prior to 12 November 2018).

Bottche 2016 \{published data only\}

Bottche M, Kuwert P, Pietrzak RH, Knaevelsrud C. Predictors of outcome of an Internet-based cognitive-behavioural therapy for post-traumatic stress disorder in older adults. Psychology and Psychotherapy 2016; 89(1):82-96.

Brief 2012 \{published data only\}

Brief D, Rubin A, Enggasser J, Roy M, Lachowicz M, Helmuth E, et al. Web-based intervention for returning veterans with risky alcohol use abstract. Alcoholism, Clinical and Experimental Research 2012; 36:347A.

Brief 2013 \{published data only\}

Brief DJ, Rubin A, Keane TM, Enggasser JL, Roy M, Helmuth E, et al. Web intervention for OEF/OIF veterans with problem drinking and PTSD symptoms: a randomized clinical trial. Journal of Consulting Psychology 2013; 81:890-900.

Daggett 2014 \{published data only\}

Daggett V, Bakas T, Murray L, Woodward-Hagg H, Williams JG, Maddox K, et al. Feasibility and satisfaction with the VETeranS Compensate, Adapt, REintegrate (VETS-CARE) intervention. Brain Injury 2014; 28(5-6):554-5. 
Elbers 2013 \{published data only\}

Elbers NA, Akkermans AJ, Cuijpers P, Bruinvels DJ. Effectiveness of a web-based intervention for injured claimants: a randomized controlled trial. Trials 2013; 14:227.

\section{Gawlytta 2017 \{published data only\}}

Gawlytta R, Niemeyer H, Bottche M, Scherag A, Knaevelsrud C, Rosendahl J. Internet-based cognitive-behavioural writing therapy for reducing post-traumatic stress after intensive care for sepsis in patients and their spouses (REPAIR): results of two pilot cases. Infection 2017; 45:S59.

\section{Hirai 2005 \{published data only\}}

Hirai M, Clum GA. An Internet-based self-change programme for traumatic event related fear, distress and maladaptive coping. Journal of Traumatic Stress 2005; 18:631-6.

\section{Hirai 2012 \{published data only\}}

Hirai M, Skidmore ST, Clum GA, Dolma S. An investigation of the efficacy of online expressive writing for trauma-related psychological distress in Hispanic individuals. Behavior Therapy 2012; 43:812-24.

\section{Kersting 2011 \{published data only\}}

Kersting A, Kroker K, Schlicht S, Baust K, Wagner B. Efficacy of cognitive behavioral Internet-based therapy in parents after the loss of a child during pregnancy: pilot data from a randomized controlled trial. Archives of Women's Mental Health 2011; 14:465-77.

\section{Kersting 2013 \{published data only\}}

Kersting A, Dolemeyer R, Steinig J, Walter F, Kroker K, Baust K, et al. Brief Internet-based intervention reduces posttraumatic stress and prolonged grief in parents after the loss of a child during pregnancy: a randomized controlled trial. Psychotherapy and Psychosomatics 2013; 82:372-81.

\section{Knaevelsrud 2006 \{published data only\}}

Knaevelsrud C, Maercker A. Does the quality of the working alliance predict treatment outcome in online psychotherapy for traumatized patients? Journal of Medical Internet Research 2006; 8(4):e31.

Knaevelsrud C, Maercker A. Internet-based treatment for PTSD reduces distress and facilitates the development of a strong therapeutic alliance: a randomized controlled clinical trial. BMC Psychiatry 2007; 7:13.

Knaevelsrud C, Wagner B, Maercker A. Internet-based treatment of posttraumatic stress: does the working alliance predict longterm therapy outcome. In: Joint Meeting of the European \& UK Chapters of the Society for Psychotherapy Research; 2005 March 2-5; Lausanne. Society for Psychotherapy Research UK \& European Chapters, 2005:14-5.

\section{Knaevelsrud 2010a \{published data only\}}

Knaevelsrud C, Liedl A, Maercker A. Posttraumatic growth, optimism and openness as outcomes of a cognitive-behavioural intervention for posttraumatic stress reactions. Journal of Health Psychology 2010; 15:1030-8.

\section{Knaevelsrud 2010b \{published data only\}}

Knaevelsrud C, Maercker A. Long-term effects of an Internetbased treatment for posttraumatic stress. Cognitive Behaviour Therapy 2010; 39:72-7.

. Effects of an Internet-based intervention for posttraumatic stress disorder. clinicaltrials.gov/ct2/show/NCT01508377 Date first received: 22 December 2011

\section{Knaevelsrud 2017 \{published data only\}12608000259347}

Knaevelsrud C, Bottche M, Pietrzak RH, Freyberger HJ, Kuwert P. Efficacy and feasibility of a therapist-guided Internet-based intervention for older persons with childhood traumatization: a randomized controlled trial. American Journal of Geriatric Psychiatry 2017; 25(8):878-88.

\section{Lange 2001 \{published data only\}}

Lange A, van de Ven JP, Schrieken B, Emmelkamp PM. Interapy. Treatment of posttraumatic stress through the Internet: a controlled trial. Journal of Behavior Therapy and Experimental Psychiatry 2001; 32(2):73-90.

\section{Lange 2003 \{published data only\}}

Lange A, Rietdijk D, Hudcovicova M, van de Ven JP, Schrieken B, Emmelkamp PM. Interapy: a controlled randomized trial of the standardized treatment of posttraumatic stress through the Internet. Journal of Consulting and Clinical Psychology 2003; 71(5):901-9.

Lange A, van de Ven JP, Schrieken B. Interapy: treatment of post-traumatic stress via the Internet. Cognitive Behaviour Therapy 2003; 32(3):110-24.

\section{Maercker 2004 \{published data only\}}

Maercker A, Knaevelsrud C, Wagner B, Lange A. Intherapy: an Internet-based treatment trial of CBT of traumatic stress. In: Twentieth Annual Meeting, International Society for Traumatic Stress Studies; 2004 November 14-18; New Orleans (LA). 2004.

\section{McGlinchey 2014 \{published data only\}}

McGlinchey R, Rosenblatt A, Mercado R, Esterman M, DeGutis J. Internet-based cognitive training enhances attention and functional outcomes in OEF/OIF/OND veterans abstract. In: 10th World Congress on Brain Injury of the International Brain Injury Association; 2014 March 19-22; San Francisco (CA). 2014:624-5.

\section{NCT01508377 \{published data only\}}

. Effects of an Internet-based intervention for posttraumatic stress disorder. clinicaltrials.gov/ct2/show/NCT01508377 Date first received: 22 December 2011.

\section{NCT01552278 \{published data only\}}

. Enhancing cognitive function and reintegration in Iraq and Afghanistan veterans with PTSD using computer-based cognitive training. clinicaltrials.gov/show/NCT01552278 Date first received: 15 March 2010.

\section{NCT01678196 \{published data only\}}

. Helping families help veterans with PTSD and alcohol abuse: an RCT of VA-CRAFT. clinicaltrials.gov/ct2/show/NCT01678196 Date first received: 3 September 2012. 


\section{NCT01710943 \{published data only\}}

. Web-based CBT for recent veterans experiencing problems with trauma symptoms or alcohol/drug use. ClinicalTrials.gov/ show/NCT01710943 Date first received: 19 October 2012.

\section{NCT01760213 \{published data only\}}

. Brief Internet based treatment for PTSD. ClinicalTrials.gov/ show/NCT01760213 Date first received: 4 January 2013.

\section{NCT01891734 \{published data only\}}

. Enhancing delivery of problem solving therapy using SmartPhone technology. Available from clinicaltrials.gov/show/ NCT01891734 Date first received: 25 June 2013.

\section{Nieminen 2016 \{published data only\}}

Nieminen K, Berg I, Frankenstein K, Vilta L, Larsson K, Persson U, et al. Internet-provided cognitive behaviour therapy of posttraumatic stress symptoms following childbirth - a randomized controlled trial. Cognitive Behaviour Therapy 2016; 45(4):287-306.

\section{Possemato 2010 \{published data only\}}

Possemato KA, Ouimette PC, Geller PA. Internet-based expressive writing for kidney transplant recipients: effects on posttraumatic stress and quality of life. Traumatology 2010; 16:49-54.

\section{Possemato 2011 \{published data only\}}

Possemato K, Ouimette P, Knowlton P. A brief self-guided telehealth intervention for post-traumatic stress disorder in combat veterans: a pilot study. Journal of Telemedicine and Telecare 2011; 17:245-50.

\section{Sayer 2009 \{published data only\}}

- Military to civilian: RCT of an intervention to promote postdeployment reintegration. clinicaltrials.gov/show/ NCT00640445 Date first received: 18 March 2008.

Sayer NA, Orazem R, Frazier P, Noorbaloochi S, Schnurr P, Litz B, et al. Military to Civilian: RCT of a web-based expressive writing intervention. In: Military Health Research Forum; 2009 August 31-September 3; Kansas City (MO). 2009.

\section{Solzbacher 2012 \{published data only\}}

Solzbacher S, Rueddel H. Effectiveness of E-therapy skill training in inpatients with anorexia nervosa, PTSD or personality disorders. Psychosomatic Medicine 2012; 74(3):A28-9.

\section{Steinmetz 2012 \{published data only\}}

Steinmetz SE, Benight CC, Bishop SL, James LE. My disaster recovery: a pilot randomized controlled trial of an Internet intervention. Anxiety, Stress, and Coping 2012; 25:593-600.

\section{Stockton 2014 \{published data only\}}

Stockton H, Joseph S, Hunt N. Expressive writing and posttraumatic growth: an Internet-based study. Traumatology $2014 ; 20: 75-83$
Wagner 2007 \{published data only\}

Wagner B, Knaevelsrud C, Maercker A. Post-traumatic growth and optimism as outcomes of an Internet-based intervention for complicated grief. Cognitive Behaviour Therapy 2007; 36:156-61.

Wagner 2012 \{published data only\}

Wagner B, Brand J, Schulz W, Knaevelsrud C. Online working alliance predicts treatment outcome for posttraumatic stress symptoms in Arab war-traumatized patients. Depression and Anxiety 2012; 29:646-51.

\section{Wang 2013 \{published data only\}}

Wang Z, Wang J, Maercker A. Chinese my trauma recovery, a Web-based intervention for traumatized persons in two parallel samples: randomized controlled trial. Journal of Medical Internet Research 2013; 15:e213.

Zernicke 2014 \{published data only\}

Zernicke KA, Campbell TS, Speca M, McCabe-Ruff K, Flowers S, Carlson LE. A randomized wait-list controlled trial of feasibility and efficacy of an online mindfulness-based cancer recovery program: the eTherapy for cancer applying mindfulness trial. Psychosomatic Medicine 2014; 76(4):257-67.

\section{References to ongoing studies}

\section{ACTRN12611000989943 \{published data only\}}

. A comparison of Internet-based cognitive behavioural therapy for posttraumatic stress disorder with and without exposure: a randomized controlled trial. www.anzctr.org.au/ ACTRN12611000989943.aspx Date first received: 16 September 2011.

\section{ACTRN12614001213639 \{published data only\}}

. Internet treatment for posttraumatic stress disorder (PTSD). www.anzctr.org.au/ACTRN12614001213639.aspx Date first received: 18 November 2014

Allen AR, Newby JM, Smith J, Andrews G. Internet-based cognitive behavioural therapy (iCBT) for posttraumatic stress disorder versus waitlist control: study protocol for a randomised controlled trial. Trials 2015; $\mathbf{1 6 : 5 4 4}$

\section{ACTRN12616000956404 \{published data only\}}

Internet-based intervention for posttraumatic stress disorder (PTSD) in soldiers: exploring mechanisms of treatment outcome. www.anzctr.org.au/ACTRN12616000956404.aspx Date first received: 20 July 2016.

\section{DRKS00010245 \{published data only\}}

. Evaluation of web-based cognitive and behavioural therapeutic components and its change mechanisms for Arab people with posttraumatic stress disorder. www.drks.de/ DRKS00010245 Date first received: 30 March 2016.

\section{ISRCTN13697710 \{published data only\}}

. A study of trauma-focused online guided self help versus trauma-focused cognitive behavioural therapy for posttraumatic stress disorder. isrctn.com/ISRCTN13697710 Date first received: 21 December 2016 


\section{ISRCTN16806208 \{published data only\}}

. A randomised controlled trial of therapist-assisted online psychological therapies for post-traumatic stress disorder. isrctn.com/ISRCTN16806208 Date first received: 5 January 2018.

\section{Lehavot 2017 \{published data only\}}

Lehavot K, Litz B, Millard SP, Hamilton AB, Sadler A, Simpson T. Study adaptation, design, and methods of a web-based PTSD intervention for women Veterans. Contemporary Clinical Trials 2017; 53:68-79.

\section{McLean 2017 \{published data only\}}

McLean CP, Rauch SA, Foa EB, Sripada RK, Tannahill HS, Mintz J, et al. Design of a randomized controlled trial examining the efficacy and biological mechanisms of web-prolonged exposure and present-centered therapy for PTSD among active-duty military personnel and veterans. Contemporary Clinical Trials 2017; 64:41-8.

\section{NCT02556645 \{published data only\}}

. A comparison of web-prolonged exposure (Web-PE) and present-centered therapy (PCT) for PTSD among active-duty military personnel. ClinicalTrials.gov/show/NCT02556645 Date first received: 22 September 2015.

\section{NCT02611843 \{published data only\}}

. Peer supported web-based CBT for OEF/OIF veterans with PTSD and substance misuse. ClinicalTrials.gov/show/ NCT02611843 Date first received: 23 November 2015.

NCT02929979 \{published data only\}

. Cognitive remediation for alcohol use disorder and posttraumatic stress disorder. ClinicalTrials.gov/show/ NCT02929979 Date first received: 11 October 2016.

\section{NCT03208738 \{published data only\}}

. Pilot evaluation of the VetChange mobile app for veterans with PTSD who engage in problem drinking. ClinicalTrials.gov/show/ NCT03208738 Date first registered: 5 July 2017.

\section{NTR6912 \{published data only\}}

. Addition of the SUPPORT Coach in PTSD treatment. www.trialregister.nl/trialreg/admin/rctview.asp?TC=6912 Date first received: 22 December 2017.

\section{Additional references}

\section{ACPMH 2007}

Phoenix Australia - Centre for Posttraumatic Mental Health. Australian Guidelines for the treatment of adults with acute stress disorder and posttraumatic stress disorder, 2007. phoenixaustralia.org/resources/ptsd-guidelines/ (accessed 6 June 2017).

\section{Alonso 2014}

Alonso J, Angermeyer MC, Lépine JP. The European Study of the Epidemiology of Mental Disorders (ESEMeD) project: an epidemiological basis for informing mental health policies in Europe.. Acta Psychiatrica Scandinavica 2014; 109(s420):5-7.

\section{Andersson 2005}

Andersson G, Bergstrom J, Carlbring P, Lindefors N. The use of the Internet in the treatment of anxiety disorders. Current Opinion in Psychiatry 2005; 18:73-7.

\section{Andersson 2009}

Andersson G. Using the Internet to provide cognitive behaviour therapy. Behaviour Research and Therapy 2009; 47(3):175-80.

\section{APA 1980}

American Psychiatric Association. Diagnostic and Statistical Manual of Mental Disorders (DSM-III). Washington (DC): American Psychiatric Association, 1980.

\section{APA 1987}

American Psychiatric Association. Diagnostic and Statistical Manual of Mental Disorders (DSM-IIIR). 3rd edition. Washington (DC): American Psychiatric Association, 1987.

\section{APA 2000}

American Psychiatric Association. Diagnostic and Statistical Manual of Mental Disorders (DSM IV). 4th edition. Washington (DC): American Psychiatric Association, 2000.

\section{APA 2004}

Ursano RJ, Bell C, Eth S, Friedman M, Norwood A, Pfefferbaum B, et al, Work Group on ASD and PTSD , Steering Committee on Practice Guidelines. Practice guidelines for the treatment of patients with acute stress disorder and posttraumatic stress disorder. American Journal of Psychiatry 2004; 161(11 Suppl):3-31.

\section{APA 2013}

American Psychiatric Association. Diagnostic and Statistical Manual of Mental Disorders (DSM-5). 5th edition. Washington (DC): APA, 2013.

\section{Beck 1961}

Beck AT, Ward CH. An inventory for measuring depression. Archives of General Psychiatry 1961; 4:561-71.

\section{Beck 1993}

Beck AT, Steer RA. Beck Anxiety Inventory Manual. San Antonio (TX): The Psychological Corporation, 1993.

\section{Bisson 2013}

Bisson JI, Roberts NP, Andrew M, Cooper R, Lewis CE. Psychological therapies for chronic posttraumatic stress disorder (PTSD) in adults. Cochrane Database of Systematic Reviews 2013, Issue 12. [DOI: 10.1002/14651858.CD003388.pub4]

\section{Blake 1995}

Blake DD, Weathers FW, Nagy LM, Kaloupek DG, Gusman FD, Charney DS. The development of a clinician-administered PTSD scale. Journal of Traumatic Stress 1995; 8:75-90.

\section{Bradley 2005}

Bradley R, Green J, Russ E, Dutra L, Westen D. A multidimensional meta-analysis of psychotherapy for PTSD. American Journal of Psychiatry 2005; 162(2):214-27. 


\section{Brewin 2000}

Brewin CR, Andrews B, Valentine JD. Meta-analysis of risk factors for posttraumatic stress disorder in trauma-exposed adults. Journal of Consulting and Clinical Psychology 2000; 68(5):748-66.

\section{Bryant 2003}

Bryant RA, Moulds ML, Guthrie RM, Dang ST, Nixon RDV. Imaginal exposure alone and imaginal exposure with cognitive restructuring in treatment of posttraumatic stress disorder. Journal of Consulting \& Clinical Psychology 2003; 71(4):706-12.

\section{Creamer 2001}

Creamer M, Burgess P, McFarlane AC. Post-traumatic stress disorder: findings from the Australian national survey of mental health and well-being. Psychological Medicine 2001; 31:1237-47.

\section{Creamer 2004}

Creamer M, Forbes D, Phelps A, Humphreys L. Treating traumatic stress: conducting imaginal exposure. Phoenix Australia - Centre for Posttraumatic Mental Health 2004.

\section{Cuijpers 2008}

Cuijpers P, van Straten A, Andersson G. Internet-administered cognitive behavior therapy for health problems: a systematic review. Journal of Behavioral Medicine 2008; 31(2):169-77.

\section{Cuijpers 2010}

Cuijpers P, Donker T, van Straten A, Li J. Is guided self-help as effective as face-to-face psychotherapy for depression and anxiety disorders? A systematic review and meta-analysis of comparative outcome studies. Psychological Medicine 2010; 40(12):1943-57.

\section{Ferry 2010}

Ferry F, Bolton D, Bunting B, O'Neill S, Murphy S, Devine B. Economic impact of post traumatic stress in Northern Ireland. Northern Ireland Centre for Trauma and Transformation and University of Ulster Psychology Research Institute :2010.

\section{Foa 2007}

Foa E, Hembree E, Rothbaum BO. Prolonged Exposure Therapy for PTSD: Emotional Processing of Traumatic Experiences Therapist Guide. Oxford, UK: Oxford University Press, 2007.

\section{Foa 2008}

Foa E, Keane TM, Friedman MJ, Cohen JA. Effective Treatments for PTSD: Practice Guidelines From the International Society for Traumatic Stress Studies. New York (NY): Guilford Press, 2008.

\section{Gega 2004}

Gega L, Marks I, Mataix-Cols D. Computer-aided CBT self-help for anxiety and depressive disorders: experience of a London clinic and future directions. Journal of Clinical Psychology 2004; 60(2):147-57.

\section{Gillies 2012}

Gillies D, Taylor F, Gray C, O'Brien L, D'Abrew N. Psychological therapies for the treatment of post-traumatic stress disorder in children and adolescents. Cochrane
Database of Systematic Reviews 2012, Issue 12. [DOI:

10.1002/14651858.CD006726.pub2]

\section{GRADEpro GDT [Computer program]}

GRADE Working Group, McMaster University GRADEpro GDT. Version (accessed 15 May 2017). Hamilton (ON): GRADE Working Group, McMaster University, 2015.

\section{Hetrick 2010}

Hetrick SE, Purcell R, Garner B, Parslow R. Combined pharmacotherapy and psychological therapies for posttraumatic stress disorder (PTSD). Cochrane Database of Systematic Reviews 2010, Issue 7. [DOI: 10.1002/14651858.CD007316.pub2]

\section{Higgins 2011}

Higgins JP, Green S, . Cochrane Handbook for Systematic Reviews of Interventions Version 5.1 (updated March 2011). The Cochrane Collaboration, 2011. Available from www.handbook.cochrane.org.

\section{IAPT 2010}

Baguley C, Farrand P, Hope R, Leibowitz J, Lovell K, Lucock M, et al, . Good practice guidance on the use of self-help materials within IAPT services, 2010. www.iapt.nhs.uk/silo/files/goodpractice-guidance-on-the-use-of-selfhelp-materials-within-iaptservices.pdf) (accessed 6 June 2017).

\section{Lewis 2010}

Lewis C, Bisson JI. Health economics of web based mental health interventions to mitigate the effects of trauma. In: Brunet A, Ashbaugh A, Herbert C, editors(s). The Internet in the Aftermath of Trauma. Amsterdam: IOS Press, 2010.

\section{Lewis 2012}

Lewis C, Pearce J, Bisson JI. Systematic review of the efficacy, cost effectiveness and acceptability of self-help interventions for individuals diagnosed with an anxiety disorder. British Journal of Psychiatry 2012; 200(1):15-21.

\section{Lewis 2013}

Lewis C, Roberts NR, Vick T, Bisson JI. Development of a guided self-help (GSH) program for the treatment of mild to moderate post-traumatic stress disorder (PTSD). Depression and Anxiety 2013; 30(11):1121-8.

\section{Mayo-Wilson 2013}

Mayo-Wilson E, Montgomery P. Media-delivered cognitive behavioural therapy and behavioural therapy (self-help) for anxiety disorders in adults. Cochrane Database of Systematic Reviews 2013, Issue 9. [DOI: 10.1002/14651858.CD005330.pub4]

\section{NCCMH 2005}

National Collaborating Centre for Mental Health (NCCMH). Posttraumatic stress disorder (PTSD): the management of PTSD in adults and children in primary and secondary care (CG26). Gaskell and the British Psychological Society, London 2005.

\section{Newman 2003}

Newman MG, Erickson T, Przeworski A, Dzus E. Self-help and minimal contact therapies for anxiety disorders: is human 
contact necessary for therapeutic efficacy? Journal of Clinical Psychology 2003; 59(3):251-74.

\section{NICTT 2008}

Northern Ireland Centre for Trauma and Transformation and the Psychology Research Institute. A study of the epidemiology of trauma-related disorders and qualitative investigation of the impact of trauma on the individual. Trauma, Health and Conflict in Northern Ireland. Ulster, Ireland 2008.

\section{Norris 2003}

Norris FH, Murphy AD, Baker CK, Perilla JL, Rodriguez FG, Rodriguez JDJG. Epidemiology of trauma and posttraumatic stress disorder in Mexico. Journal of Abnormal Psychology 2003; 112(4):646

\section{Olthuis 2015}

Olthuis JV, Watt MC, Bailey K, Hayden JA, Stewart SH. Therapistsupported Internet cognitive behavioural therapy for anxiety disorders in adults. Cochrane Database of Systematic Reviews 2015, Issue 3. [DOI: 10.1002/14651858.CD011565]

\section{Ozer 2003}

Ozer EJ, Lipsey TL, Weiss DS. Predictors of posttraumatic stress disorder and symptoms in adults: a meta-analysis. Psychological Bulletin 2003; 129(1):52-73.

\section{Review Manager 2014 [Computer program]}

The Nordic Cochrane Centre, The Cochrane Collaboration Review Manager 5 (RevMan 5). Version 5.3. Copenhagen: The Nordic Cochrane Centre, The Cochrane Collaboration, 2014.

\section{Roberts 2009}

Roberts NP, Kitchiner NJ, Kenardy J, Bisson JI. Multiple session early psychological interventions for the prevention of posttraumatic stress disorder. Cochrane Database of Systematic Reviews 2009, Issue 3. [DOI: 10.1002/14651858.CD006869.pub2]

\section{Roberts 2010}

Roberts NP, Kitchiner NJ, Kenardy J, Bisson JI. Early psychological interventions to treat acute traumatic stress symptoms. Cochrane Database of Systematic Reviews 2010, Issue 3. [DOI: 10.1002/14651858.CD007944.pub2]

\section{CHARACTERISTICS OF STUDIES}

Characteristics of included studies [ordered by study ID]

\section{Rose 2002}

Rose S, Bisson J, Wessely S. Psychological debriefing for preventing post traumatic stress disorder (PTSD). Cochrane Database of Systematic Reviews 2002, Issue 2. [DOI: 10.1002/14651858.CD000560]

\section{Spek 2007}

Spek V, Cuijpers P, Nyklícek I, Riper H, Keyzer J, Pop V. Internetbased cognitive behaviour therapy for symptoms of depression and anxiety: a meta-analysis. Psychological Medicine 2007; 37(3):319-28.

\section{Stein 2006}

Stein DJ, Ipser JC, ,. Pharmacotherapy for post traumatic stress disorder (PTSD). Cochrane Database of Systematic Reviews 2006, Issue 1. [DOI: 10.1002/14651858.CD002795.pub2]

\section{Weathers 2013}

Weathers FW, Litz BT, Keane TM, Palmieri PA, Marx BP, Schnurr PP. The PTSD Checklist for DSM-5 (PCL-5), 2013. www.ptsd.va.gov/professional/assessment/adult-sr/ptsdchecklist.asp (accessed prior to 24 November 2018).

\section{WHO 1979}

World Health Organization. International Statistical Classification of Diseases and Related Health Problems (ICD-9) Mental Disorders. Geneva: World Health Organization, 1979.

\section{WHO 1992}

World Health Organization. International Statistical Classification of Diseases and Related Health Problems (ICD-10) Classification of Mental and Behavioural Disorders. Geneva: World Health Organization, 1992.

\section{Wilson 2012}

Wilson JP, Friedman MJ, Lindy JD, . Treating Psychological Trauma and PTSD. New York (NY): Guilford Press, 2012.

\section{References to other published versions of this review Lewis 2015}

Lewis C, Roberts NP, Bethell A, Bisson JI. Internet-based cognitive and behavioural therapies for post-traumatic stress disorder (PTSD) in adults. Cochrane Database of Systematic Reviews 2015, Issue 5. [DOI: 10.1002/14651858.CD011710]

\section{Engel 2015}

\section{Study characteristics}

Methods Randomised controlled trial

Participants

Sample size: 80

Diagnosis: DSM-IV PTSD 
Method of diagnosis: CAPS

Trauma type: military

Age (mean): intervention: 36.2 (SD 7.75) years; usual care: 36.7 (SD 9.75) years

Sex: $18.75 \%$ women; $81.25 \%$ men

Location: US

Comorbidities: no structured screening for comorbid conditions

Adjunctive therapy: excluded

Adjunctive medication: stable

Unemployment: not reported

University education: $62.8 \%$

Exclusion criteria: active engagement in trauma-focused mental health treatment in previous 2 months; recent history of failed specialty mental health treatment for PTSD or associated condition; acute psychosis, psychotic episode, or psychotic disorder diagnosed within the past 2 years; active substance dependence in past year; active suicidal or homicidal ideation within past 2 months; currently taking antipsychotic or mood-stabilising medication; unstable administration schedule or dosing of any antidepressant, anxiolytic, or sedative-hypnotic during last month; or acute or unstable physical illness.

Baseline PTSD: all participants met diagnostic criteria for PTSD.

Baseline PCL scores (mean): intervention: 58.00 (SD 9.95); usual care: 54.48 (SD 11.23)

Interventions

Internet programme based on trauma-focused CBT versus treatment as usual

\section{Experimental arm}

Duration: 6 weeks (with access for 8 weeks, or 10 in exceptional circumstances)

Treatment protocol

- Variant of non-trauma focused CBT and stress inoculation training approaches.

- Nurse-guided.

- Participants asked to log on the secure website 3 times each week for 6 weeks and complete various homework activities.

- Educational information about PTSD, stress, and trauma, as well as common comorbid problems and symptoms (e.g. depression and survivor guilt).

- Information on strategies to manage anger and promote better sleep hygiene, as well as indepth information on how to perform and practice deep, slow diaphragmatic breathing, and simple progressive muscle relaxation.

- Cognitive reframing techniques, such as how to challenge unhelpful thought patterns and alter selftalk to manage demanding situations effectively.

- Hierarchy of difficult and avoided situations that triggered deployment memories or were generally stressful.

- At each login, participants were asked to make ratings of their PTSD symptoms and a global rating of their level of depression. Each login consisted of a new set of educational materials followed by a homework assignment to practice new techniques and skills learned. Broken up into 5 units, these assignments promoted stress-reduction skills, self-monitoring, application of stress management techniques to each stress hierarchy item, processing and coping with a particularly salient or intense traumatic memory as well as very significant current day stressors, and relapse prevention.

- Participants required to complete homework to continue subsequent content.

- Each login intended to take 15-30 minutes and homework assignments another 30 minutes. 
Therapist contact: participants encouraged to contact nurse for assistance if needed. Nurses were able to access a private portion of the DESTRESS-PC website where they could monitor compliance and symptom levels.

Type of contact: participants encouraged nurse for assistance if needed. Nurse had access to a private portion of the DESTRESS-PC website to monitor compliance and symptom levels.

Automated contact: none

Type of therapist: nurse

Treatment fidelity: unclear

\section{Comparator arm}

Duration: 6 weeks

- Optimised usual PTSD care consisted of usual primary care PTSD treatment augmented with low intensity care management, feedback to the primary care provider, and training of the clinic providers in management of PTSD.

- Designed to approximate the level of PTSD care normally provided in primary care while incorporating the non-specific treatment elements of the DESTRESS intervention.

Time points for assessment: pretreatment, post-treatment, 6 weeks, 12 weeks, 18 weeks
PCL-C; PHQ-8; PHQ-15

Notes Funding source: unclear

Declarations of interest among the primary researchers: unclear

\section{Risk of bias}

\begin{tabular}{lll}
\hline Bias & Authors' judgement & Support for judgement \\
\hline $\begin{array}{l}\text { Random sequence genera- } \\
\text { tion (selection bias) }\end{array}$ & Low risk & $\begin{array}{l}\text { Quote: "Randomization was performed centrally from Walter Reed's Deploy- } \\
\text { ment Health Clinical Center with subsequent notification of the site investiga- } \\
\text { tor and the site's DESTRESS Nurse. Stratification of randomization by health- } \\
\text { care site and gender was done to facilitate exploratory analyses on trial out- } \\
\text { comes [20]; and employed a random permuted blocking scheme (1:1 alloca- } \\
\text { tion ratio with variable block size of four to six) [21] was employed." }\end{array}$ \\
\hline $\begin{array}{l}\text { Allocation concealment } \\
\text { (selection bias) }\end{array}$ & Unclear risk & Not reported
\end{tabular}

\begin{tabular}{|c|c|c|}
\hline $\begin{array}{l}\text { Blinding of participants } \\
\text { and personnel (perfor- }\end{array}$ & High risk & $\begin{array}{l}\text { Quote: "the nature of the intervention precluded blinding of providers and } \\
\text { participants." }\end{array}$ \\
\hline
\end{tabular}

mance bias)

All outcomes

\begin{tabular}{lll}
\hline $\begin{array}{l}\text { Blinding of outcome as- } \\
\text { sessment (detection bias) } \\
\text { All outcomes }\end{array}$ & Low risk & Quote: "we blinded the outcome raters in this trial." \\
\hline $\begin{array}{l}\text { Incomplete outcome data } \\
\text { (attrition bias) }\end{array}$ & Low risk & $\begin{array}{l}\text { Quote: "Because the PROC MIXED procedure uses all of the available data and } \\
\text { restricted maximum likelihood to estimate the unknown variance-covariance } \\
\text { parameters, it ensures a robust handling of missing follow-up data [29]. We ex- } \\
\text { amined the effects of time, treatment group, and the treatment group by time } \\
\text { interaction on the primary outcome (PCL scores) for the entire sample and for } \\
\text { the two types of recruitment sites (VA, DoD) separately." }\end{array}$
\end{tabular}


$14 / 80$ dropped out

$\begin{array}{ll}\begin{array}{l}\text { Selective reporting (re- } \\ \text { porting bias) }\end{array} & \text { Low risk } \\ \text { pected outcomes, including those that were prespecified. }\end{array}$

Other bias Low risk

Ivarsson 2014

\section{Study characteristics}

\begin{tabular}{|c|c|}
\hline Methods & Randomised controlled trial \\
\hline \multirow[t]{16}{*}{ Participants } & Sample size: 62 \\
\hline & Diagnosis: DSM-IV PTSD \\
\hline & Method of recruitment: adverts in local and national newspapers \\
\hline & Method of diagnosis: CAPS by telephone \\
\hline & Trauma type: various (apart from childhood abuse) \\
\hline & Age (mean): immediate treatment: 44.8 (SD 11.2) years; delayed treatment: 47.2 (SD 12.2) years \\
\hline & Sex: $82.3 \%$ women; $17.7 \%$ men \\
\hline & Location: Sweden \\
\hline & Comorbidities: no structured screening for comorbid conditions \\
\hline & Adjunctive therapy: excluded \\
\hline & Adjunctive medication: $25.8 \%$ (stable for 3 months) \\
\hline & Unemployment: $8.1 \%$ \\
\hline & University education: $56.5 \%$ \\
\hline & $\begin{array}{l}\text { Exclusion criteria: imminent suicide risk; concurrent psychological treatment; presence of alcohol } \\
\text { abuse; ongoing trauma, or trauma within the past } 3 \text { months; symptoms following childhood abuse. }\end{array}$ \\
\hline & Baseline PTSD: all participants met diagnostic criteria for PTSD. \\
\hline & $\begin{array}{l}\text { Baseline IES-R Scores (mean): immediate treatment: } 54.65 \text { (SD 13.16); delayed treatment: } 54.87 \text { (SD } \\
\text { 15.48) }\end{array}$ \\
\hline
\end{tabular}

Interventions

\section{Internet programme based on trauma-focused CBT versus treatment as usual}

\section{Experimental arm}

Duration: 8 weeks

Treatment protocol

- 8 text-based modules delivered once a week.

- Included psychoeducation, anxiety coping skill training (controlled breathing and conditioned relaxation, with skills training to facilitate trauma exposure, and some information on sleep); exposure (imaginal exposure carried out through writing and reading trauma narratives); and cognitive restructuring (psychoeducation about common thoughts and beliefs related to trauma and their impact on emotions and behaviour, especially avoidance behaviour). Participants given an opportunity to make 
a personal commitment for change through a treatment contract. Final module aimed at relapse prevention and maintenance of progress.

- Mostly text and images with a "basic layout."

- All modules accompanied by written homework assignments sent to therapist once a week.

- New modules only made available once previous one had been completed.

Therapist contact: once a week and occasional reminders via website.

Type of contact: encrypted web-service

Automated contact: none

Type of therapist: clinical psychology students (towards the end of course)

Treatment fidelity: weekly supervision with an experienced clinical psychologist to monitor fidelity

\section{Comparator arm}

Duration: 8 weeks

Participants in treatment group who did not finish all modules within 8 weeks were able to continue using the website without therapist support after post-treatment data had been collected.

Treatment protocol

- Voluntarily answering questions on well-being, stress, and sleep on a weekly basis. Participants not required to answer questions and were told that this would not affect their later treatment.

- Weekly questions were neutral to minimise spontaneous trauma writing.

- Clinician monitored responses for suicidal ideation and answered questions about trial.

Therapist contact: kept to a minimum. Therapist screened responses for suicidal ideation and was available to answer questions on the trial.

\begin{tabular}{ll}
\hline Outcomes & Time points for assessment: pretreatment, post-treatment, 1-year follow-up \\
& Primary outcome: IES-R, PDS \\
& Secondary outcome: BDI-II, BAI, QOLI, CGI-I \\
\hline Notes & Funding source: grant from Linköping University \\
& Declarations of interest among primary researchers: unclear
\end{tabular}

\section{Risk of bias}

\begin{tabular}{|c|c|c|}
\hline Bias & Authors' judgement & Support for judgement \\
\hline $\begin{array}{l}\text { Random sequence genera- } \\
\text { tion (selection bias) }\end{array}$ & Low risk & $\begin{array}{l}\text { Quote: "Randomization was conducted by an individual who was not other- } \\
\text { wise involved in the research project, using an online true random number ser- } \\
\text { vice (www.random.org)." }\end{array}$ \\
\hline $\begin{array}{l}\text { Allocation concealment } \\
\text { (selection bias) }\end{array}$ & Unclear risk & $\begin{array}{l}\text { Quote: "Randomization was conducted by an individual who was not other- } \\
\text { wise involved in the research project, using an online true random number ser- } \\
\text { vice (www.random.org)." }\end{array}$ \\
\hline $\begin{array}{l}\text { Blinding of participants } \\
\text { and personnel (perfor- } \\
\text { mance bias) } \\
\text { All outcomes }\end{array}$ & High risk & Impossible to blind participants or therapists. \\
\hline
\end{tabular}

\begin{tabular}{|c|c|c|}
\hline $\begin{array}{l}\text { Blinding of outcome as- } \\
\text { sessment (detection bias) }\end{array}$ & Low risk & $\begin{array}{l}\text { Quote: "The post-treatment interviewers were blind to participant status (i.e. } \\
\text { treatment or control)." }\end{array}$ \\
\hline
\end{tabular}

Internet-based cognitive and behavioural therapies for post-traumatic stress disorder (PTSD) in adults (Review) 
Ivarsson 2014 (Continued)

All outcomes
Blinding impossible at 1-year follow-up since there was no longer a control group.

\section{Incomplete outcome data Low risk} (attrition bias)

All outcomes
Quote: "All analyses, both the pre- to post-treatment and the pre-treatment to one year follow-up analysis, made use of all available data from all randomized participants, following the principle of intention-to-treat. Prior to conducting primary analyses, the missing data assumption was tested by exploring associations between baseline characteristics and the presence of missing data."

\begin{tabular}{lll}
\hline $\begin{array}{l}\text { Selective reporting (re- } \\
\text { porting bias) }\end{array}$ & Low risk & $\begin{array}{l}\text { Study protocol not available but it was clear that the published reports includ- } \\
\text { ed all expected outcomes, including those that were prespecified. }\end{array}$ \\
\hline Other bias & High risk & Evaluated by the originators of the intervention. \\
\hline
\end{tabular}

\section{Knaevelsrud 2015}

\section{Study characteristics}

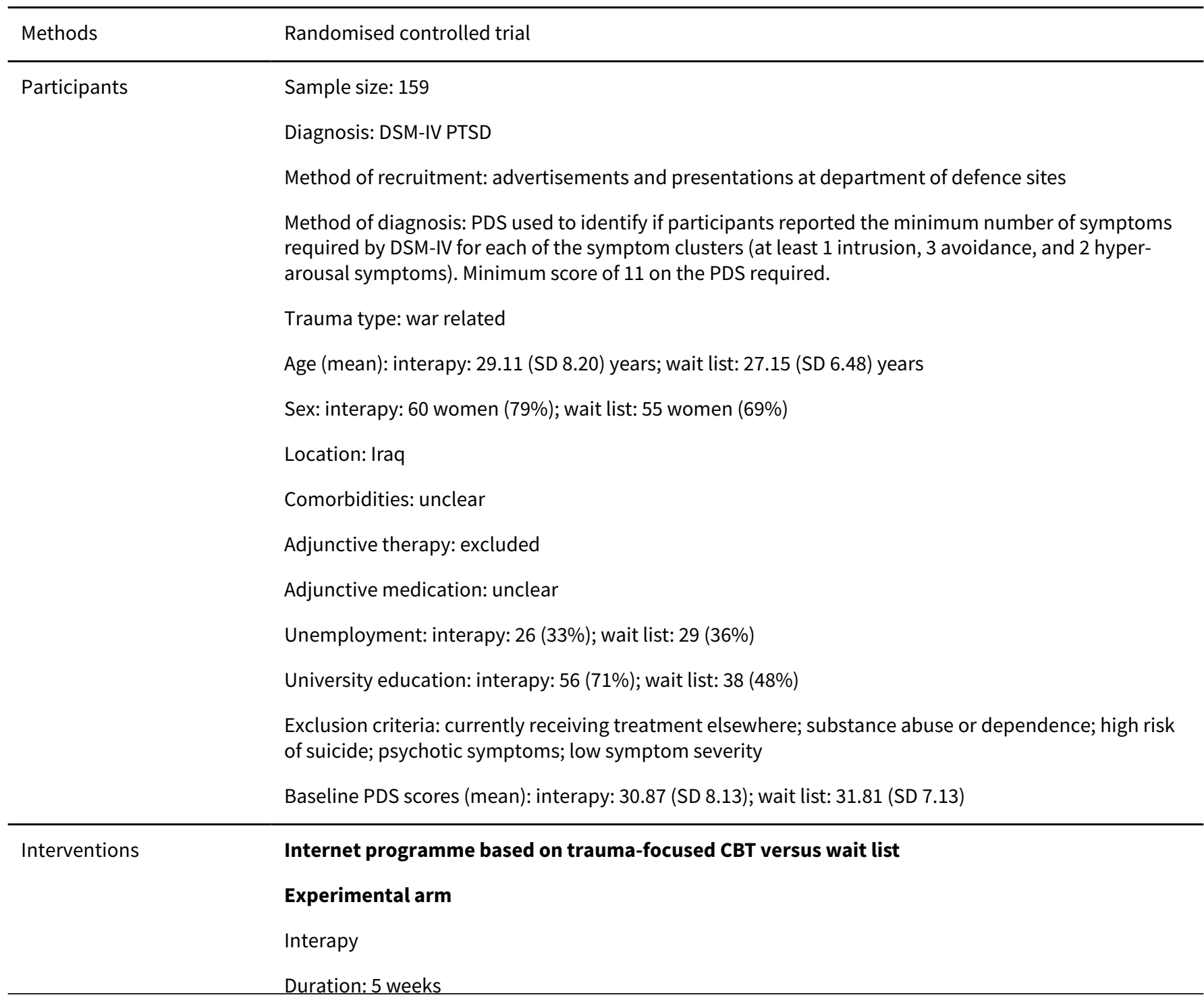


- 2 weekly structured writing activities assigned each week over period of 5 weeks.

- 3 treatment phases: self-confrontation with the traumatic event, cognitive restructuring, and social sharing.

Type of contact: short homework reminder by e-mail, if no response contacted by phone; exact method of communication unclear.

Automated contact: unknown, not reported

Type of therapist: native speaking psychotherapists

Treatment fidelity: weekly supervision sessions, either face-to-face or via Skype

\section{Comparator arm}

Duration: 6 weeks

Treatment protocol: participants were on a wait list for 6 weeks and then received the intervention.

Therapist contact: none

\begin{tabular}{ll}
\hline Outcomes & PDS; HSCL-25; EUROHIS-QUOL \\
\hline Notes & Funding source: unclear \\
& Declarations of interest among the primary researchers: unclear \\
\hline
\end{tabular}

\section{Risk of bias}

\begin{tabular}{lll}
\hline Bias & Authors' judgement & Support for judgement \\
\hline $\begin{array}{l}\text { Random sequence genera- } \\
\text { tion (selection bias) }\end{array}$ & Low risk & $\begin{array}{l}\text { Quote: "Randomisation was based on a computer-generated randomisation } \\
\text { list." }\end{array}$ \\
\hline $\begin{array}{ll}\text { Allocation concealment } \\
\text { (selection bias) }\end{array}$ & Unclear risk & Not reported \\
\hline $\begin{array}{l}\text { Blinding of participants } \\
\text { and personnel (perfor- } \\
\text { mance bias) }\end{array}$ & High risk & \\
\begin{tabular}{l} 
All outcomes \\
\hline
\end{tabular} & & $\begin{array}{l}\text { Quote: "researchers and psychotherapists were not masked to the interven- } \\
\text { tion." }\end{array}$ \\
\hline
\end{tabular}

\begin{tabular}{|c|c|c|}
\hline $\begin{array}{l}\text { Blinding of outcome as- } \\
\text { sessment (detection bias) } \\
\text { All outcomes }\end{array}$ & Low risk & Comment: self-reported measures \\
\hline
\end{tabular}

\begin{tabular}{|c|c|c|}
\hline $\begin{array}{l}\text { Incomplete outcome data } \\
\text { (attrition bias) }\end{array}$ & High risk & ITT analysis BUT missing data $>30 \%$ \\
\hline
\end{tabular}

All outcomes

\begin{tabular}{|c|c|c|}
\hline $\begin{array}{l}\text { Selective reporting (re- } \\
\text { porting bias) }\end{array}$ & Low risk & $\begin{array}{l}\text { Study protocol available (Registered Trial (p.2) - Australian New Zealand Clin- } \\
\text { ical Trial Registry) and the published reports included all expected outcomes, } \\
\text { including those that were prespecified. }\end{array}$ \\
\hline
\end{tabular}

Other bias Low risk Study appeared free from other sources of bias.


Krupnick 2017

\section{Study characteristics}

\begin{tabular}{|c|c|}
\hline Methods & Randomised controlled trial \\
\hline \multirow[t]{15}{*}{ Participants } & Sample size: 34 \\
\hline & Diagnosis: DSM-IV PTSD \\
\hline & Method of recruitment: clinician referral \\
\hline & Method of diagnosis: PCL-M with cut-off score 50 \\
\hline & Trauma type: military \\
\hline & Age (mean): WIRED: 35.44 years; WIRED+TAU: 44.75 years (SDs not reported) \\
\hline & Sex: $8.8 \%$ women; $91.2 \%$ men \\
\hline & Location: US \\
\hline & Comorbidities: no structured screening for comorbid conditions \\
\hline & Adjunctive therapy: a chart review revealed that several participants received adjunctive treatment. \\
\hline & Adjunctive medication: $50 \%$ \\
\hline & Unemployment: not reported \\
\hline & University education: not reported \\
\hline & $\begin{array}{l}\text { Exclusion criteria: current substance dependence, acute suicidality, psychosis, gross cognitive impair- } \\
\text { ment, current participation in CBT }\end{array}$ \\
\hline & Baseline PCL-M scores (mean item score): WIRED: 3.6 (SD 0.3); WIRED+TAU: 3.9 (SD 0.4) \\
\hline
\end{tabular}

\section{Internet programme based on trauma-focused CBT versus treatment as usual}

\section{Experimental arm}

WIRED (based on Interapy)

Duration: 10 sessions

Treatment protocol

- 10 writing sessions (adapted from Interapy).

- First 4 sessions confronted the trauma.

- Next 4 focused on cognitive restructuring of maladaptive thoughts about the experience.

- Final 2 sessions emphasises leave-taking and social sharing.

Therapist contact: as required. A short response and further instructions sent after each writing session.

Type of contact: online

Automated contact: none

Type of therapist: psychologist

Treatment fidelity: unclear

\section{Comparator arm}

TAU 
- A chart review after completion of the study showed that 4 participants began and 1 completed a course of cognitive processing therapy.

- 8 participants received antidepressant medication.

- 1 participant received 13 sessions of acupuncture.

\begin{tabular}{ll}
\hline Outcomes & PCL-M (mean item scores); PHQ-9 (could not be entered into meta-analysis) \\
\hline Notes & $\begin{array}{l}\text { Funding source: grant from Telemedicine and Advanced Technology Research Center, U.S. Department } \\
\text { of Defense. }\end{array}$ \\
& Declarations of interest among the primary researchers: unclear \\
\hline
\end{tabular}

\section{Risk of bias}

\begin{tabular}{|c|c|c|}
\hline Bias & Authors' judgement & Support for judgement \\
\hline $\begin{array}{l}\text { Random sequence genera- } \\
\text { tion (selection bias) }\end{array}$ & Unclear risk & Not reported \\
\hline $\begin{array}{l}\text { Allocation concealment } \\
\text { (selection bias) }\end{array}$ & Unclear risk & Not reported \\
\hline $\begin{array}{l}\text { Blinding of participants } \\
\text { and personnel (perfor- } \\
\text { mance bias) } \\
\text { All outcomes }\end{array}$ & High risk & Impossible to blind participants or therapists. \\
\hline $\begin{array}{l}\text { Blinding of outcome as- } \\
\text { sessment (detection bias) } \\
\text { All outcomes }\end{array}$ & Low risk & $\begin{array}{l}\text { All measures administered after baseline were self-reported and completed } \\
\text { online. }\end{array}$ \\
\hline $\begin{array}{l}\text { Incomplete outcome data } \\
\text { (attrition bias) } \\
\text { All outcomes }\end{array}$ & High risk & Dropout rate $>75 \%$. Reasons for dropout not fully reported. \\
\hline $\begin{array}{l}\text { Selective reporting (re- } \\
\text { porting bias) }\end{array}$ & Low risk & $\begin{array}{l}\text { Study protocol not available but it was clear that the published reports includ- } \\
\text { ed all expected outcomes, including those that were prespecified. }\end{array}$ \\
\hline Other bias & High risk & Small sample size. Participants received other interventions during the trial. \\
\hline
\end{tabular}

Kuhn 2017

\section{Study characteristics}

\begin{tabular}{ll}
\hline Methods & Randomised controlled trial \\
\hline Participants & Sample size: 120 \\
& Diagnosis: DSM-IV PTSD \\
& Method of recruitment: advertisements through fliers, media coverage, social media and websites \\
& (Craigslist) \\
& Method of diagnosis: PCL-C \\
& Trauma type: various
\end{tabular}


Kuhn 2017 (Continued)

Age (mean): PTSD Coach: 39.43 (SD 15.16) years; wait list: 39.12 (SD 14.08) years

Sex: $69.2 \%$ women, $30.8 \%$ men

Location: US

Comorbidities: not reported

Adjunctive therapy: not reported

Adjunctive medication: not reported

Unemployment: not reported

University education: $14.2 \%$

Exclusion criteria: currently receiving treatment for PTSD

Baseline PTSD: all participants met diagnostic criteria for PTSD (no participant had a PCL score < 35).

Baseline PCL scores (mean): for PTSD Coach: 63.19 (SD 11.78); wait list: 60.59 (SD 10.24)

Interventions

Internet programme based on trauma-focused CBT versus wait list

\section{Experimental arm}

Duration: 12 weeks

Treatment protocol: PTSD Coach

PTSD Coach condition participants were instructed to download the app and use it however they would like in an attempt to mimic real use. They were e-mailed links to the post-treatment assessment 3 months later and follow-up assessment 6 months.

\section{Comparator arm}

Duration: 12 weeks

Treatment protocol: wait list

Wait list participants received no intervention during the treatment period. After the post-treatment assessment, they were told that the app being studied was PTSD Coach, that the app was available in the App Store and Google Play Store, and that they were now free to download and use it if they would like. They were e-mailed links to the post-treatment assessment 3 months later and follow-up assessment 6 months.

\begin{tabular}{|c|c|c|}
\hline \multirow[t]{3}{*}{ Outcomes } & \multicolumn{2}{|c|}{ Time points for assessment: 3 and 6 months } \\
\hline & \multicolumn{2}{|l|}{ Primary outcome: PCL } \\
\hline & \multicolumn{2}{|c|}{ Secondary outcome: PTSD symptom coping self-efficacy, PHQ-8 and B-IPF } \\
\hline \multirow[t]{2}{*}{ Notes } & \multicolumn{2}{|c|}{ Funding source: not reported } \\
\hline & \multicolumn{2}{|c|}{ Declarations of interest among the primary researchers: none } \\
\hline \multicolumn{3}{|l|}{ Risk of bias } \\
\hline Bias & Authors' judgement & Support for judgement \\
\hline $\begin{array}{l}\text { Random sequence genera- } \\
\text { tion (selection bias) }\end{array}$ & Low risk & $\begin{array}{l}\text { Quote: "randomized to conditions by the study coordinator using adaptive } \\
\text { randomization, with the probability of condition assignment changing based } \\
\text { on the assignment of participants already in the trial using www.randomiz- } \\
\text { er.com." }\end{array}$ \\
\hline
\end{tabular}


Kuhn 2017 (Continued)

\begin{tabular}{lll}
$\begin{array}{l}\text { Allocation concealment } \\
\text { (selection bias) }\end{array}$ & Unclear risk & Not reported \\
\hline $\begin{array}{l}\text { Blinding of participants } \\
\text { and personnel (perfor- }\end{array}$ & High risk & Impossible to blind participants or therapists. \\
mance bias) & & \\
All outcomes &
\end{tabular}

\begin{tabular}{lll}
\hline $\begin{array}{l}\text { Blinding of outcome as- } \\
\text { sessment (detection bias) } \\
\text { All outcomes }\end{array}$ & Low risk & Comment: self-reported measures. \\
\hline $\begin{array}{l}\text { Incomplete outcome data } \\
\begin{array}{l}\text { (attrition bias) } \\
\text { All outcomes }\end{array}\end{array}$ & Low risk & $\begin{array}{l}\text { Quote: "Following the intent-to-treat principle, data from all randomized par- } \\
\text { ticipants were analyzed, and multiple imputation was used to replace missing } \\
\text { values, with } 10 \text { imputed data sets being generated." }\end{array}$ \\
\hline
\end{tabular}

Selective reporting (re- Low risk Study protocol not available but it was clear that the published reports includporting bias) ed all expected outcomes, including those that were prespecified.

Other bias Low risk Study appeared free from other sources of bias.

Lewis 2017

\section{Study characteristics}

\begin{tabular}{|c|c|}
\hline Methods & Randomised controlled trial \\
\hline \multirow[t]{12}{*}{ Participants } & Sample size: 42 \\
\hline & Diagnosis: DSM-5 PTSD \\
\hline & $\begin{array}{l}\text { Method of recruitment: primary and secondary care clinicians referred participants in accordance with } \\
\text { a brief recruitment protocol and a checklist of inclusion criteria. Study also advertised through local } \\
\text { media and by means of posters and leaflets in high-footfall National Health Service waiting areas. } 40 \\
\text { (95\%) had been referred to the study by treating clinicians and } 2(5 \%) \text { were recruited by means of ad- } \\
\text { verts in the media. }\end{array}$ \\
\hline & Method of diagnosis: DSM-5 PTSD as measured by CAPS- 5 . \\
\hline & $\begin{array}{l}\text { Trauma type: transportation accidents ( } 9 \text { participants); witnessing a sudden, violent, or accidental } \\
\text { death (9); traumatic childbirth or stillbirth (8); sexual assault or rape (5); physical attack (4); life-threat- } \\
\text { ening illness or injury (3); serious accident (1); learning of the violent death of a loved one (1); seeing a } \\
\text { mutilated body (1); and being held hostage/detained (1). }\end{array}$ \\
\hline & $\begin{array}{l}\text { Age (mean): treatment: } 38.86 \text { (SD 11.91) years; range 20-65 years; delayed treatment: } 37.71 \text { (SD 13.8) } \\
\text { years; range } 21-64 \text { years }\end{array}$ \\
\hline & Sex: $59.5 \%$ women; $40.5 \%$ men \\
\hline & Location: UK \\
\hline & Comorbidities: not reported \\
\hline & Adjunctive therapy: excluded \\
\hline & Adjunctive medication: stable for 1 month \\
\hline & Unemployment: $16.6 \%$ \\
\hline
\end{tabular}


Lewis 2017 (Continued)

University education: $42.8 \%$

Exclusion criteria: psychosis, previous trauma-focused psychological therapy, DSM- 5 severe major depressive episode, substance dependence, inability to read and write fluent English, inability to access the Internet, change in psychotropic medication within 1-month, concurrent psychological therapy, and suicidal intent.

Baseline PTSD: all participants met diagnostic criteria for PTSD.

Baseline CAPS-5 scores (mean): immediate treatment: 35.99 (SD 6.29); delayed treatment: 37.12 (SD 6.95)

Interventions

Internet programme based on trauma-focused CBT versus wait list

\section{Experimental arm}

Duration: 8 weeks

Treatment protocol: interactive, online, guided self-help intervention, which included 8 online steps designed for delivery over 8 weeks.

- Step 1: learning about my PTSD: psychoeducational material related to PTSD, illustrated by video clips of 4 characters describing their experiences.

- Step 2: grounding myself: explanation of grounding and its uses along with descriptions and demonstrations of grounding exercises.

- Step 3: managing my anxiety: education around relaxation with learning through videos of a controlled breathing technique, deep muscular relaxation, and relaxation through imagery.

- Step 4: reclaiming my life: behavioural reactivation to help people return to previously undertaken and explore new activities.

- Step 5: coming to terms with my trauma: provides rationale for imaginal exposure, narratives of the 4 video characters are provided. The therapist helps the participant to begin writing a narrative, which they complete remotely and read every day for at least 30 minutes.

- Step 6: changing my thoughts: cognitive techniques to address associated negative appraisals.

- Step 7: overcoming my avoidance: graded in vivo exposure work to trauma-related avoided and feared situations.

- Step 8: keeping myself well: reinforces what has been learnt during the programme, provides relapse prevention measures and guidance on what to do if symptoms return.

The programme was initiated with a 1-hour session with a therapist-guide, followed by 30-minute appointments every 2 weeks.

Therapist contact: mean amount of therapist input per participant was 147.53 minutes (SD 57.01). This included a mean of 3.09 (SD 1.84) face-to-face meetings, 2.09 (SD 1.85) telephone calls, and 1.00 (SD 1.62) e-mails.

Type of contact: face-to-face, telephone, e-mail

Automated contact: not reported

Type of therapist: 3 male and 2 female therapists who were experienced in the delivery of trauma-focused CBT guided the programme. They were trained to deliver the Internet-based therapy, followed an intervention manual, and attended regular supervision meetings to maximise adherence to the manual.

Treatment fidelity: therapists attended regular supervision meetings to maximise adherence to the manual.

\section{Comparator arm}

Duration: 8 weeks

Treatment protocol: participants were on a wait list for 14 weeks and then received the intervention. 
Lewis 2017 (Continued)

Therapist contact: none

Outcomes Time points for assessment:

Treatment group: pretreatment, post-treatment (10 weeks after randomisation), 1 month (14 weeks after randomisation), and 3-month follow-up (22 weeks after randomisation).

Delayed treatment group: pretreatment, 10 weeks after randomisation, 14 weeks after randomisation, and post-treatment ( 22 weeks after randomisation)

Primary outcome: CAPS-5

Secondary outcome: BDI, BAI, AUDIT, SSQ, SDS

Notes

Funding source: Knowledge Transfer Partnership (KTP008512)

Declarations of interest among the primary researchers: undertaken as part of a Knowledge Transfer Partnership between Cardiff University and Healthcare Learning Smile-on. If the programme is marketed, royalties will be payable to Cardiff University and Cardiff and Vale University Health Board, with a proportion of these being shared with CL, NK, NR, TV, and JB. DF and VG have no conflicts of interest to disclose.

\section{Risk of bias}

\begin{tabular}{|c|c|c|}
\hline Bias & Authors' judgement & Support for judgement \\
\hline $\begin{array}{l}\text { Random sequence genera- } \\
\text { tion (selection bias) }\end{array}$ & Low risk & $\begin{array}{l}\text { Quote: "Participants were randomly assigned, through a system of sealed, } \\
\text { opaque envelopes containing an allocation code generated by an independent } \\
\text { statistician, to an immediate or delayed treatment group." }\end{array}$ \\
\hline $\begin{array}{l}\text { Allocation concealment } \\
\text { (selection bias) }\end{array}$ & Low risk & $\begin{array}{l}\text { Quote: "Participants were randomly assigned, through a system of sealed, } \\
\text { opaque envelopes containing an allocation code generated by an independent } \\
\text { statistician, to an immediate or delayed treatment group." }\end{array}$ \\
\hline $\begin{array}{l}\text { Blinding of participants } \\
\text { and personnel (perfor- } \\
\text { mance bias) } \\
\text { All outcomes }\end{array}$ & High risk & Impossible to blind participants or therapists. \\
\hline $\begin{array}{l}\text { Blinding of outcome as- } \\
\text { sessment (detection bias) } \\
\text { All outcomes }\end{array}$ & Low risk & $\begin{array}{l}\text { Quote: "A trained postdoctoral researcher who was blind to group allocation } \\
\text { carried out all assessments." }\end{array}$ \\
\hline $\begin{array}{l}\text { Incomplete outcome data } \\
\text { (attrition bias) } \\
\text { All outcomes }\end{array}$ & Low risk & $\begin{array}{l}\text { Quote: "Throughout, varying engagement in the trial and with the treatment } \\
\text { was handled using intention-to-treat principles." }\end{array}$ \\
\hline $\begin{array}{l}\text { Selective reporting (re- } \\
\text { porting bias) }\end{array}$ & Low risk & $\begin{array}{l}\text { Study protocol not available but it was clear that the published reports includ- } \\
\text { ed all expected outcomes, including those that were prespecified. }\end{array}$ \\
\hline Other bias & High risk & Small sample size. Evaluated by the originators of the intervention. \\
\hline
\end{tabular}

\section{Study characteristics}


Littleton 2016 (Continued)

Participants
Sample size: 87

Diagnosis: DSM-IV PTSD

Method of recruitment: advertisements at 4 universities/community colleges, including postings on university psychology department participant management websites, and via social media.

Method of diagnosis: PSS-SI

Trauma type: rape

Age (mean): 22 years (range 18-42 years) for whole sample

Sex: $100 \%$ women

Location: US

Comorbidities: SUD excluded

Adjunctive therapy: not reported

Adjunctive medication: not reported

Unemployment: not reported

University education: all students

Exclusion criteria: current psychotherapy; changes in psychotropic medication in past 3 months; active suicidality; DSM-IV criteria for current substance dependence.

Baseline PTSD: not reported

Baseline PSS-I scores (mean): From Survivor to Thriver: 11.2 (SD 5.8); psychoeducational website: 10.4 (SD 8.5)

Interventions

\section{Internet programme based on trauma-focused CBT with Internet psychological therapy (non-} (BT)

\section{Experimental arm}

Duration: 14 weeks

Treatment protocol: From Survivor to Thriver

From Survivor to Thriver program consisted of 9 programme modules to be completed sequentially, 1 module at a time. The program had 3 phases. The first phase (modules 1-3) was designed to provide psychoeducation about PTSD and the impact of unwanted sex, as well as introduce general distress management strategies (i.e. relaxation, grounding) and healthy coping (e.g. asking others for help, setting an action plan) skills. The second phase (modules 4-5) introduced the cognitive model and taught participants to identify distorted and unhelpful automatic thoughts and utilise the challenging questions technique to respond to these thoughts. The third phase (modules 6-9) focused on using a number of cognitive behavioural techniques (e.g. the challenging questions technique, the pros and cons technique, behavioural experiments) to address specific concerns common among women following sexual assault (e.g. difficulties with trust, self-blame for the assault). Each module contained several components, including: a video clip of the program developers discussing the topic covered in the module, a written description of the skills or techniques being introduced or utilised in the module, and, for modules 4-9, written examples of women modelling using the skills and techniques utilised in the module. The final program component included in all modules was the interactive exercises. These interactive exercises were designed either to help women think about the extent to which the particular issue raised in the module was a concern for them (e.g. the extent to which they blame themselves for the sexual assault) or to enable them to practice a described cognitive-behavioural skill or technique and receive feedback on their practice. The interactive exercises were completed asynchronously, and were designed in a question and answer format. After a participant completed the interactive exercise, her program therapist then provided written feedback to each answer provided, as well as 
embedded a video message containing more extensive feedback on the interactive exercise page in the program. Therapist feedback focused on encouraging and praising participants for their effort, expressing empathy, encouraging continued program progress, socialising participants to the cognitive mod$\mathrm{el}$, and providing assistance in challenging automatic thoughts, such as through further use of Socratic questioning.

\section{Comparator arm}

Duration: 14 weeks

Treatment protocol: psychoeducational website

The psychoeducational website contained the written informational content of the first 3 modules of the interactive program including the symptoms of PTSD, information about relaxation and grounding, and information about healthy coping strategies. The website did not contain any of the multimedia content (videos from the program developers, audio recorded relaxation exercises), or any interactive exercises. Women assigned to the psychoeducational website condition were given access to all the website content and instructed to log in to the program and utilise the content in whatever manner and however frequently they desired to do so over the course of the study.

\begin{tabular}{ll}
\hline Outcomes & Time points for assessment: 3 months \\
& Primary outcome: PSS-I \\
& Secondary outcome: CES-D, FDAS \\
\hline Notes & Funding source: not reported \\
& Declarations of interest among the primary researchers: none
\end{tabular}

\section{Risk of bias}

\begin{tabular}{lll}
\hline Bias & Authors' judgement & Support for judgement \\
\hline $\begin{array}{l}\text { Random sequence genera- } \\
\text { tion (selection bias) }\end{array}$ & Low risk & $\begin{array}{l}\text { Quote: "Participants were randomized to the interactive program or psychoe- } \\
\text { ducational website based on a computerized coin flip." }\end{array}$ \\
\hline $\begin{array}{l}\text { Allocation concealment } \\
\text { (selection bias) }\end{array}$ & Unclear risk & Not reported \\
\hline $\begin{array}{l}\text { Blinding of participants } \\
\begin{array}{l}\text { and personnel (perfor- } \\
\text { mance bias) }\end{array}\end{array}$ & High risk & Impossible to blind participants or therapists. \\
All outcomes & &
\end{tabular}

\begin{tabular}{lll}
\hline Blinding of outcome as- & High risk & Quote: "individuals completing pre-, post- and follow-up assessments were \\
sessment (detection bias) & not blind to participants' intervention condition." \\
All outcomes &
\end{tabular}

\begin{tabular}{|c|c|c|}
\hline $\begin{array}{l}\text { Incomplete outcome data } \\
\text { (attrition bias) } \\
\text { All outcomes }\end{array}$ & Low risk & $\begin{array}{l}\text { Quote: "In addition, estimates of change from pre-test to post-test and fol- } \\
\text { low-up were estimated for all participants assigned to a program, an Intent to- } \\
\text { Treat (ITT) model. For the ITT analyses, post-test and follow-up data for par- } \\
\text { ticipants who left the study was imputed using the R package. Twenty imputa- } \\
\text { tions were used with all outcome variables." }\end{array}$ \\
\hline
\end{tabular}

\begin{tabular}{|c|c|c|}
\hline $\begin{array}{l}\text { Selective reporting (re- } \\
\text { porting bias) }\end{array}$ & Low risk & $\begin{array}{l}\text { Study protocol not available but it was clear that the published reports includ- } \\
\text { ed all expected outcomes, including those that were prespecified. }\end{array}$ \\
\hline
\end{tabular}

Other bias Low risk Study appeared to be free from other sources of bias.




\section{Study characteristics}

\begin{tabular}{|c|c|}
\hline Methods & Randomised controlled trial \\
\hline \multirow[t]{16}{*}{ Participants } & Sample size: 45 \\
\hline & Diagnosis: DSM-IV PTSD \\
\hline & Method of recruitment: advertisements and presentations at department of defence sites \\
\hline & Method of diagnosis: PSS-I \\
\hline & Trauma type: combat exposure: $9 / 11$ attack on the Pentagon, or combat in Iraq or Afghanistan \\
\hline & Age (mean): Internet CBT: 38.63 (SD 9.41) years; Internet supportive counselling: 39.86 (SD 7.72) years \\
\hline & Sex: unclear \\
\hline & Location: US \\
\hline & Comorbidities: unclear \\
\hline & Adjunctive therapy: excluded \\
\hline & Adjunctive medication: unclear \\
\hline & Unemployment: unclear \\
\hline & University education: unclear \\
\hline & $\begin{array}{l}\text { Exclusion criteria: active substance dependence; current suicidal ideation; history of psychotic disor- } \\
\text { der; aged < } 21 \text { or }>65 \text { years; PTSD or depression immediately before the trauma; current psychiatric } \\
\text { treatment; marked ongoing stressors; inadequate social supports; change in medication. }\end{array}$ \\
\hline & Baseline PTSD: all participants met diagnostic criteria for PTSD. \\
\hline & Baseline PSS-I scores: Internet CBT: 26.71 (SD 9.02); Internet supportive counselling: 29.16 (SD 9.93) \\
\hline
\end{tabular}

Interventions

Internet programme based on trauma-focused CBT with Internet psychological therapy (nonCBT)

\section{Experimental arm}

Duration: 8 weeks

Treatment protocol

- Self-monitoring of situations that triggered trauma-related distress.

- Generation of a serial ordering (hierarchy) of these trigger contexts in terms of their degree of threat or avoidance.

- Stress management strategies.

- Graduated, self-guided, in vivo exposure to items from the personalised hierarchy (starting with the least threatening or least avoided item in week 3 ).

- 7 online trauma writing sessions.

- A review of progress (charts of daily symptom reports were presented), a series of didactics on relapse prevention, and the generation of a personalised plan for future challenges.

Therapist contact: initial face-to-face (2 hours, including baseline assessment); telephone (ad-lib); email (ad-lib)

Type of contact: online, e-mail, face-to-face 
Litz 2007 (Continued)

\author{
Automated contact: none \\ Type of therapist: unclear \\ Treatment fidelity: not reported
}

\title{
Comparator arm
}

Duration: 8 weeks

Treatment protocol

- Monitoring non-trauma-related concerns and online writing about these experiences.

- Psychoeducation materials were available about the psychological, emotional, and cognitive effects of trauma, but this was provided for information only, and no action was required.

- Supportive counselling group was asked to visit the website daily to report their symptoms, read about stress and stress management, and write about current concerns.

- Website allowed the participants to ask for an immediate telephone call from their therapists, and they were called periodically by their study therapist to check in on how they were doing and to answer any questions they might have about the self-help program. Through e-mail and the telephone, supportive counselling therapists were instructed to be empathic and validating, non-directive and supportive, and to focus on non-trauma-related present-day concerns. In week 8 , participants in the supportive counselling arm were asked to plan ways of using what they learned in the course of the therapy from that point forward, and to plan for future stressors; they also were shown graphs of the course of their progress (symptom reporting).

Therapist contact: initial face-to-face ( 2 hours, including baseline assessment), telephone (ad-lib), email (ad-lib)

Type of contact: online, e-mail, face-to-face

Automated contact: none

Type of therapist: unclear

\begin{tabular}{|c|c|c|}
\hline \multirow[t]{3}{*}{ Outcomes } & \multicolumn{2}{|c|}{ Time points for assessment: pretreatment, post-treatment, 3-month follow-up, and 6-month follow-up } \\
\hline & \multicolumn{2}{|c|}{ Primary outcome: PSS-I } \\
\hline & \multicolumn{2}{|c|}{ Secondary outcome: BDI, BAI } \\
\hline \multirow[t]{2}{*}{ Notes } & \multicolumn{2}{|c|}{ Funding source: supported by grant from NIHM (MH66589) } \\
\hline & \multicolumn{2}{|c|}{ Declarations of interest among the primary researchers: none } \\
\hline \multicolumn{3}{|l|}{ Risk of bias } \\
\hline Bias & Authors' judgement & Support for judgement \\
\hline $\begin{array}{l}\text { Random sequence genera- } \\
\text { tion (selection bias) }\end{array}$ & Unclear risk & Not reported \\
\hline $\begin{array}{l}\text { Allocation concealment } \\
\text { (selection bias) }\end{array}$ & Unclear risk & Not reported \\
\hline $\begin{array}{l}\text { Blinding of participants } \\
\text { and personnel (perfor- } \\
\text { mance bias) } \\
\text { All outcomes }\end{array}$ & High risk & Impossible to blind participants or therapists. \\
\hline
\end{tabular}


Litz 2007 (Continued)

Blinding of outcome as- Low risk Quote: "The study therapists conducted the baseline assessments in order sessment (detection bias)

All outcomes

Incomplete outcome data High risk

(attrition bias)

All outcomes

Selective reporting (re- Low risk
porting bias)

Other bias

High risk to initially establish rapport. Clinicians blind to study arm conducted the follow-up evaluations."
Authors stated that they performed ITT analyses, but only completer analyses were reported.

Miner 2016

\section{Study characteristics}

\begin{tabular}{|c|c|}
\hline Methods & Randomised controlled trial \\
\hline \multirow[t]{16}{*}{ Participants } & Sample size: 49 \\
\hline & Diagnosis: DSM-IV PTSD \\
\hline & Method of recruitment: advertisements through fliers and websites \\
\hline & Method of diagnosis: PCL-C \\
\hline & Trauma type: various \\
\hline & Age (mean): total: 45.7 (SD 13.9) years. Age range not reported by group. \\
\hline & Sex: $81.6 \%$ women, $18.4 \%$ men \\
\hline & Location: US \\
\hline & Comorbidities: not reported \\
\hline & Adjunctive therapy: not reported \\
\hline & Adjunctive medication: not reported \\
\hline & Unemployment: not reported \\
\hline & University education: not reported \\
\hline & Exclusion criteria: currently receiving treatment for PTSD \\
\hline & $\begin{array}{l}\text { Baseline PTSD: all participants met diagnostic criteria for PTSD (no participant had a PCL score lower } \\
\text { than the recommended range (i.e. } 30-35 \text { ). }\end{array}$ \\
\hline & Baseline PSS-I scores (mean): Internet CBT: 63.00 (SD 11.28); wait list: 59.33 (SD 11.34) \\
\hline \multirow[t]{3}{*}{ Interventions } & Internet programme based on trauma-focused CBT versus wait list \\
\hline & Experimental arm \\
\hline & Duration: 4 weeks \\
\hline
\end{tabular}


Miner 2016 (Continued)

Treatment protocol: PTSD Coach

Participants given the app and instructed to use it however they would like for the following month. No specific training, instructions for use, or suggestions of how PTSD Coach might be helpful were provided in attempt to represent real-world use. Participants completed the post-condition assessment 1 month later.

\section{Comparator arm}

Duration: 4 weeks

Treatment protocol: wait list

No intervention over 1 month. Participants completed the post-condition assessment 1 month later. Upon completion of the post-condition assessment, participants received the PTSD Coach.

\begin{tabular}{ll}
\hline Outcomes & Time points for assessment: 4 weeks \\
& Primary outcome: $P C L$ \\
& Secondary outcome: acceptability, feasibility \\
\hline Notes & Funding source: not reported \\
& Declarations of interest among the primary researchers: none
\end{tabular}

\section{Risk of bias}

\begin{tabular}{lll}
\hline Bias & Authors' judgement & Support for judgement \\
\hline $\begin{array}{l}\text { Random sequence genera- } \\
\text { tion (selection bias) }\end{array}$ & Unclear risk & Quote: "Participants were randomly assigned with an equal chance." \\
& & Comment: insufficient reporting of methods to determine risk of bias. \\
\hline $\begin{array}{l}\text { Allocation concealment } \\
\text { (selection bias) }\end{array}$ & Unclear risk & Not reported \\
\hline $\begin{array}{l}\text { Blinding of participants } \\
\text { and personnel (perfor- } \\
\text { mance bias) } \\
\text { All outcomes }\end{array}$ & High risk & Impossible to blind participants or therapists. \\
\hline
\end{tabular}

Blinding of outcome as- Low risk Comment: all outcomes self-reported.

sessment (detection bias)

All outcomes

\section{Incomplete outcome data Low risk} (attrition bias)

All outcomes

\begin{abstract}
Quote: "The condition (PTSD Coach vs. waitlist) by time (baseline and post condition) interaction was used to estimate an effect size and assess if there was significant differential change in PCL scores using intention-to-treat (ITT) and completer analyses. For the ITT analysis multiple imputation with $10 \mathrm{im}$ puted data sets was used to replace missing PCL values at the post-condition $(n=5 ; 10.2 \%)$ and follow-up $(n=9 ; 18.4 \%)$ assessments and ranges and averages of statistics across these imputed datasets are presented."
\end{abstract}

Comment: ITT analysis and missing data $<20 \%$.

\begin{tabular}{lll}
$\begin{array}{l}\text { Selective reporting (re- } \\
\text { porting bias) }\end{array}$ & Low risk & $\begin{array}{l}\text { Study protocol not available but it was clear that the published reports includ- } \\
\text { ed all expected outcomes, including those that were prespecified. }\end{array}$ \\
\hline Other bias & Low risk & Study appeared free from other sources of bias.
\end{tabular}




\section{Study characteristics}

\begin{tabular}{|c|c|}
\hline Methods & Randomised controlled trial \\
\hline \multirow[t]{16}{*}{ Participants } & Sample size: 42 \\
\hline & Diagnosis: DSM-IV PTSD \\
\hline & $\begin{array}{l}\text { Method of recruitment: recruitment from a website that offers participation in trials, adverts in local } \\
\text { media. }\end{array}$ \\
\hline & Method of diagnosis: MINI administered via telephone \\
\hline & Trauma type: various \\
\hline & Age (mean): I-CBT: 43.0 (SD 15.2) years; wait list: 42.0 (SD 10.4) years \\
\hline & Sex: $81 \%$ women; $19 \%$ men \\
\hline & Location: Australia \\
\hline & Comorbidities: unclear \\
\hline & Adjunctive therapy: excluded \\
\hline & Adjunctive medication: stable for 1 month \\
\hline & Unemployment: 40\% \\
\hline & University education: unclear \\
\hline & $\begin{array}{l}\text { Exclusion criteria: currently experiencing a psychotic mental illness; severe symptoms of depression; } \\
\text { currently highly dissociative; current CBT. }\end{array}$ \\
\hline & Baseline PTSD: all participants met diagnostic criteria for PTSD. \\
\hline & Baseline PCL-C scores (mean): I-CBT: 60.78 (SD 10.03); wait list: 57.00 (SD 9.69) \\
\hline
\end{tabular}

\section{Internet programme based on trauma-focused CBT versus wait list}

\section{Experimental arm}

Duration: 8 weeks

Treatment protocol

- Lesson 1: education about the prevalence, symptoms, and treatment of PTSD, including an explanation of the functional relationship between symptoms.

- Lesson 2: instructions about controlling physical symptoms including dearousal strategies.

- Lesson 3: basic principles of cognitive therapy, including strategies for monitoring and challenging thoughts.

- Lesson 4: education and guidelines about practicing graded exposure.

- Lesson 5: education and guidelines about practicing imaginal exposure, using repeated written exposure, audio-recording, or both, and repeatedly listening to the recording.

- Lesson 6: education and guidelines about challenging dysfunctional beliefs, including trauma related beliefs.

- Lesson 7: information about relapse prevention and constructing relapse prevention plans.

Therapist contact (mean): 103.91 (SD 96.53) minutes

Type of contact: telephone, e-mail, forum 
Type of therapist: clinical psychologist

Treatment fidelity: not reported

\section{Comparator arm}

Duration: 8 weeks

Treatment protocol: wait list

\begin{tabular}{ll}
\hline Outcomes & Time points for assessment: pretreatment, post-treatment, and 3-month follow-up \\
& Primary outcome: PCL-C \\
& Secondary outcome: PHQ-9, GAD-7, SDS \\
\hline Notes & Funding source: supported by a research fellowship from the New South Wales Institute of Psychiatry. \\
& $\begin{array}{l}\text { Declarations of interest among the primary researchers: contract grant sponsor: New South Wales Insti- } \\
\text { tute of Psychiatry }\end{array}$ \\
\hline
\end{tabular}

\section{Risk of bias}

\section{Bias Authors' judgement Support for judgement}

Random sequence genera- Low risk
tion (selection bias)

Quote: "Forty-four applicants met all inclusion criteria and were randomized via a true randomization process (www.random.org), generated by an independent person."

\begin{tabular}{lll}
\hline $\begin{array}{l}\text { Allocation concealment } \\
\text { (selection bias) }\end{array}$ & Unclear risk & Not reported \\
\hline
\end{tabular}

Blinding of participants High risk Impossible to blind participants or therapists.
and personnel (perfor-
mance bias)
All outcomes

\begin{tabular}{lll}
\hline $\begin{array}{l}\text { Blinding of outcome as- } \\
\text { sessment (detection bias) }\end{array}$ & High risk & $\begin{array}{l}\text { Quote: "Treatment group participants had a similar diagnostic assessment at } \\
\text { three-month follow-up. These assessments were conducted by JS and KS, who } \\
\text { All outcomes }\end{array}$ \\
\hline
\end{tabular}

Incomplete outcome data High risk ITT analyses using LOCF.

(attrition bias)

All outcomes

\begin{tabular}{|c|c|c|}
\hline $\begin{array}{l}\text { Selective reporting (re- } \\
\text { porting bias) }\end{array}$ & Low risk & $\begin{array}{l}\text { Study protocol not available but it was clear that the published reports includ } \\
\text { ed all expected outcomes, including those that were prespecified. }\end{array}$ \\
\hline
\end{tabular}

\begin{tabular}{ll}
\hline Other bias & Small sample size \\
& $\begin{array}{l}\text { Recruitment ceased after } 4 \text { weeks due to staff availability. Did not meet the re- } \\
\text { cruitment target (indicated by power calculation) of } 26 \text { per group. } \\
\text { Evaluated by the originators of the intervention. } \\
\text { Time-since-trauma not recorded, so unsure about the requirement for the } \\
\text { trauma to have been at least } 1 \text { month previously. }\end{array}$
\end{tabular}


AUDIT: Alcohol Use Disorders Identification Test; BAI: Beck Anxiety Inventory; BDI-II: Beck Depression Inventory II; B-IPF: Brief-Inventory of Psychosocial Functioning; CAPS: Clinician Administered PTSD Scale; CBT: cognitive behavioural therapy; CES-D: Center for Epidemiologic Studies Depression Scale; CGI-I: Clinical Global Impressions - Improvement; DSM-IV: Diagnostic and Statistical Manual of Mental Disorders; FDAS: Food \& Drug Analytical Services; GAD-7: Generalized Anxiety Disorder 7; HSCL-25: ; I-CBT: Internet-based cognitive behavioural therapy; IES-R: Impact of Event Scale - Revised; ITT: intention to treat; LOCF: last observation carried forward; MINI: Mini-International Neuropsychiatric Interview; PCL: PTSD Checklist; PCL-C: PTSD Checklist - Civilian Version; PCL-M: PTSD Checklist - Military Version; PDS: Posttraumatic Diagnostic Scale; PHQ-9: Patient Health Questionnaire-9; PSS-I: PTSD Symptom Scale - Interview; PTSD: post-traumatic stress disorder; QOLI: Quality of Life Inventory; SD: standard deviation; SDS: Shehan Disability Scale; SSQ: Social Support Questionnaire; TAU: treatment as usual; WIRED: Warriors Internet Recovery \& EDucation.

Characteristics of excluded studies [ordered by study ID]

\begin{tabular}{|c|c|}
\hline Study & Reason for exclusion \\
\hline Bahena 2016 & No formal diagnosis of DSM/ICD PTSD. \\
\hline Bishop 2012 & Reduction in PTSD symptoms was not the main aim of the intervention. \\
\hline Blankenship 2013 & No formal diagnosis of DSM/ICD PTSD. \\
\hline Bottche 2016 & No formal diagnosis of DSM/ICD PTSD. \\
\hline Brief 2012 & No formal diagnosis of DSM/ICD PTSD. \\
\hline Brief 2013 & No formal diagnosis of DSM/ICD PTSD. \\
\hline Daggett 2014 & No formal diagnosis of DSM/ICD PTSD. \\
\hline Elbers 2013 & No formal diagnosis of DSM/ICD PTSD. \\
\hline Gawlytta 2017 & Not an RCT. \\
\hline Hirai 2005 & No formal diagnosis of DSM/ICD PTSD. \\
\hline Hirai 2012 & No formal diagnosis of DSM/ICD PTSD. \\
\hline Kersting 2011 & No formal diagnosis of DSM/ICD PTSD. \\
\hline Kersting 2013 & No formal diagnosis of DSM/ICD PTSD. \\
\hline Knaevelsrud 2006 & No formal diagnosis of DSM/ICD PTSD. \\
\hline Knaevelsrud 2010a & No formal diagnosis of DSM/ICD PTSD. \\
\hline Knaevelsrud 2010b & No formal diagnosis of DSM/ICD PTSD. \\
\hline Knaevelsrud 2017 & No formal diagnosis of DSM/ICD PTSD. \\
\hline Lange 2001 & No formal diagnosis of DSM/ICD PTSD. \\
\hline Lange 2003 & No formal diagnosis of DSM/ICD PTSD. \\
\hline Maercker 2004 & No formal diagnosis of DSM/ICD PTSD. \\
\hline McGlinchey 2014 & No formal diagnosis of DSM/ICD PTSD. \\
\hline NCT01508377 & No formal diagnosis of DSM/ICD PTSD. \\
\hline
\end{tabular}




\begin{tabular}{|c|c|}
\hline Study & Reason for exclusion \\
\hline NCT01552278 & Reduction in PTSD symptoms was not the main aim of the intervention. \\
\hline NCT01678196 & No formal diagnosis of DSM/ICD PTSD. \\
\hline NCT01710943 & Participants required to screen positive for PTSD or alcohol/drug use. \\
\hline NCT01760213 & No formal diagnosis of DSM/ICD PTSD. \\
\hline NCT01891734 & No formal diagnosis of DSM/ICD PTSD. \\
\hline Nieminen 2016 & Only $25 \%$ of the sample met diagnostic criteria for PTSD at baseline. \\
\hline Possemato 2010 & A brief self-guided telehealth intervention for PTSD in combat veterans: a pilot study. \\
\hline Possemato 2011 & A brief self-guided telehealth intervention for PTSD in combat veterans: a pilot study. \\
\hline Sayer 2009 & No formal diagnosis of DSM/ICD PTSD. \\
\hline Solzbacher 2012 & No formal diagnosis of DSM/ICD PTSD. \\
\hline Steinmetz 2012 & No formal diagnosis of DSM/ICD PTSD. \\
\hline Stockton 2014 & Reduction in PTSD symptoms was not the main aim of the intervention. \\
\hline Wagner 2007 & No formal diagnosis of DSM/ICD PTSD. \\
\hline Wagner 2012 & No formal diagnosis of DSM/ICD PTSD. \\
\hline Wang 2013 & No formal diagnosis of DSM/ICD PTSD. \\
\hline Zernicke 2014 & No formal diagnosis of DSM/ICD PTSD. \\
\hline
\end{tabular}

DSM: Diagnostic and Statistical Manual of Mental Disorders; ICD: International Classification of Diseases; PTSD: post-traumatic stress disorder; RCT: randomised controlled trial.

Characteristics of ongoing studies [ordered by study ID]

\section{ACTRN12611000989943}

\begin{tabular}{ll}
\hline Study name & $\begin{array}{l}\text { A comparison of Internet-based cognitive behavioural therapy for posttraumatic stress disorder } \\
\text { with and without exposure: a randomized controlled trial. }\end{array}$ \\
\hline Methods & $\begin{array}{l}\text { Randomised controlled trial using a list generated prior to the study via a software program } \\
\text { (www.random.org), at another site, in another country. The list will then be transcribed and details } \\
\text { transferred to sealed envelopes. }\end{array}$ \\
\hline Participants & Inclusion criteria \\
- PTSD DSM-IV \\
- Internet + printer access \\
- Australian resident \\
Exclusion criteria \\
- Psychotic disorder
\end{tabular}


ACTRN12611000989943 (Continued)

- Current or planned psychological treatment during study duration

- Change in medications during last 1 month or intended change during study duration

- Actively suicidal

- Highly dissociative

Interventions

\section{Experimental arm}

Clinician-guided I-CBT with exposure

Participants will complete 6 lessons of Internet-based treatment about management of symptoms of PTSD. 1 lesson will be completed every 7-10 days over 8 weeks.

\section{Comparator arm}

Clinician-guided I-CBT minus exposure

Participants will complete 4 lessons of Internet-based treatment about management of symptoms of PTSD using the same protocol with 2 lessons about exposure removed. 1 lesson will be completed every $7-14$ days over 8 weeks.

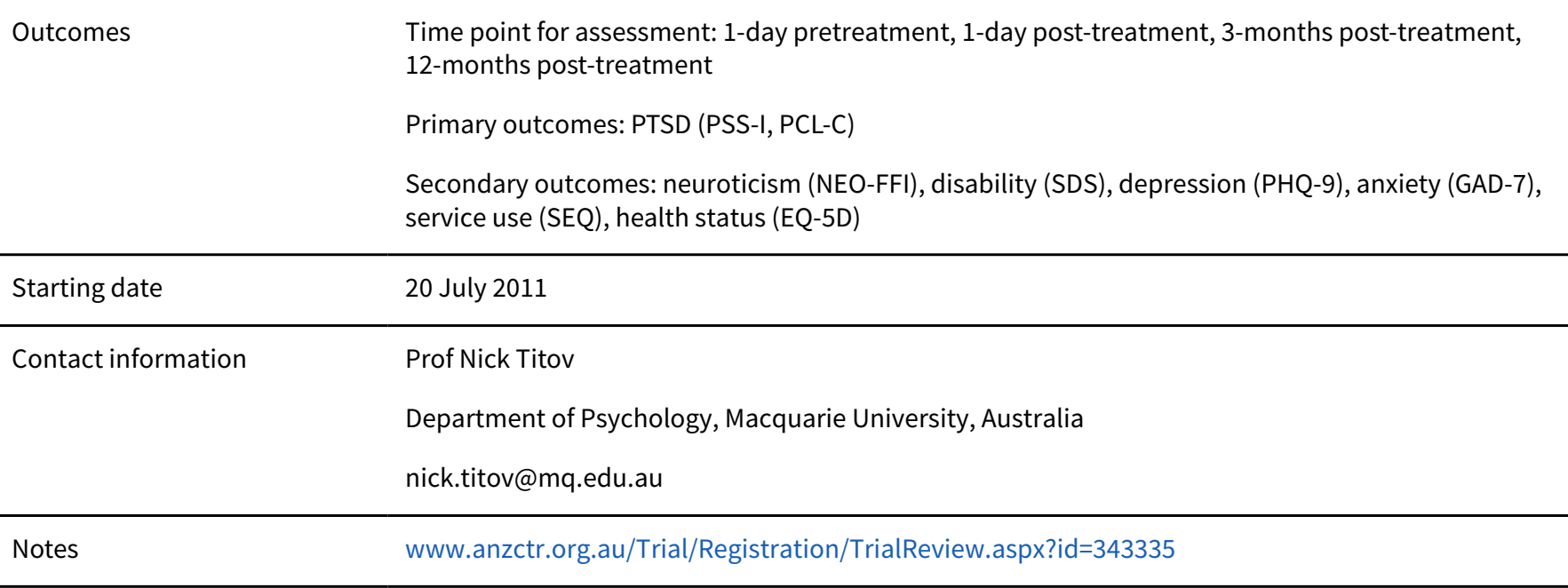

Study name Internet treatment for posttraumatic stress disorder (PTSD).

Methods

Randomised controlled trial using a list generated prior to the study. For allocation concealment, a staff member not involved in the clinical trial will generate the sequence using computer software and place each choice in a sequentially numbered, opaque sealed and stapled envelope.

\section{Participants}

Inclusion criteria

- Self-identified as having PTSD/experienced a major trauma

- Meet criteria for PTSD as determined by MINI

- Australian residential status

- Aged $\geq 18$ years

- Access to a computer, Internet, and printer

- If taking medication, must have been taking the same dose for $\geq 1$ month and not intend to change that dose during the course of the programme

- Prepared to provide name, telephone number, and address, and to provide the name and address of a local general practitioner

- Willing to provide written informed consent 
- Does not meet PTSD diagnostic criteria

- Trauma occurrence within the past 4 weeks

- Currently participating in CBT or other treatment for trauma or PTSD

- Currently highly dissociative (indicated by score $\geq 40$ on the DES)

- Current or pending medicolegal proceedings associated with the reported trauma

- Applying for or receiving Workers Compensation associated with their trauma or PTSD

- Regularly using illicit drugs or regularly consuming more than 3 standard drinks a day

- Current regular use of benzodiazepines

- Currently experiencing a psychotic mental illness or bipolar disorder

- Severe symptoms of depression (defined as a total score $\geq 22$ or responding at least ' 2 ' to Question 9 (suicidal ideation) on PHQ-9

\section{Interventions}

\section{Experimental arm}

The Internet PTSD program comprises 6 lessons completed over 10 weeks. 1 lesson will be completed every 7-14 days with a minimum of 5 days between lessons and a maximum of 14 days.

\section{Comparator arm}

Wait list control. Participants remain on the wait list until the immediate treatment group has completed their treatment and post-treatment assessment (11 weeks). At that time (11 weeks), the wait list control group will be offered the same Internet treatment program for PTSD.

\begin{tabular}{|c|c|}
\hline \multirow[t]{3}{*}{ Outcomes } & $\begin{array}{l}\text { Time point for assessment: 1-week post-treatment, 3-months post-treatment, 6-months post-treat- } \\
\text { ment }\end{array}$ \\
\hline & Primary outcomes: PTSD (PCL-C) \\
\hline & Secondary outcomes: PHQ-9, K10, CEQ, SDS, IUS-12, ERQ, WHODAS-II \\
\hline Starting date & 14 September 2014 \\
\hline \multirow[t]{3}{*}{ Contact information } & Prof Gavin Andrews \\
\hline & Clinical Research Unit for Anxiety and Depression, 390 Victoria St, Darlinghurst, Australia \\
\hline & gavina@unsw.edu.au \\
\hline Notes & www.anzctr.org.au/Trial/Registration/TrialReview.aspx?id=366115 \\
\hline
\end{tabular}

\section{ACTRN12616000956404}

Study name Internet-based intervention for posttraumatic stress disorder (PTSD) in soldiers: exploring mecha-
nisms of treatment outcome.

Methods Randomised controlled trial. Central randomisation by computer software using a random number generator.

Inclusion criteria
$\begin{aligned} & \text { Treatment group/wait list control group } \\ & \text { - German-speaking soldiers (active and out of duty) of the German Armed Forces } \\ & \text { - PTSD } \\ & \text { - Must have access to the Internet }\end{aligned}$




\section{Healthy controls group}

- German-speaking soldiers of the German Armed Forces

- Experienced a potentially traumatic event or who have never experienced a potentially traumatic event

Exclusion criteria

Treatment group/wait list control group

- People with schizophrenia, schizotypal and delusional disorders (ICD-10: F20-F29)

- Suicidal patients

- People with acute manic episode

- People with acute substance abuse or dependence

- People receiving concurrent psychotherapeutic treatment

Healthy controls group

- People fulfil criteria of PTSD or any other mental disorder

\section{Experimental arm}

Therapist-guided Internet-based intervention delivered via an online platform lasts 5 weeks and includes 10 structured writing assignments. Participants complete 2 assignments per week. Each assignment requires approximately 45 minutes of writing time.

\section{Comparator arm}

The wait list intervention group receives the same treatment as described above after an initial waiting period of 6 weeks. Participants in this group are seen for the initial assessment and pass through 1 additional assessment before the beginning of the treatment phase. The treatment procedure hereafter is identical to the protocol above.

\section{Outcomes}

Time point for assessment: post-treatment, 3-month follow-up, 6-month follow-up, and 12-month follow-up

Primary outcomes: PCL-5, CAPS

Secondary outcomes: PHQ, GAD-7, SWLS, DERS, PTCI. PTGI, CSS

\begin{tabular}{ll}
\hline Starting date & 22 August 2016 \\
\hline Contact information & Prof Christine Knaevelsrud \\
& Freie Universitaet Berlin \\
& christine.knaevelsrud@fu-berlin.de \\
\hline Notes & www.anzctr.org.au/Trial/Registration/TrialReview.aspx?id=370924 \\
\hline
\end{tabular}

\begin{tabular}{ll}
\hline Methods & Randomised controlled trial \\
\hline Participants & Inclusion criteria
\end{tabular}


DRKS00010245 (Continued)

- Arabic-speaking

- Aged > 18 years

- History of torture or trauma

- PTSD symptoms

- Internet access during the treatment period

- Personal e-mail account

Exclusion criteria

- Substance abuse or dependence

- Psychotic symptoms

- Acute suicidal tendency

- Current psychotherapeutic treatment

- Changes in psychotropic medication in the last 6 weeks

Interventions

Experimental arm 1

Web-delivered exposure-focused therapy

Experimental arm 2

Web-delivered cognitive online therapy

\section{Comparator arm}

Untreated (wait list)

\begin{tabular}{ll}
\hline Outcomes & Time point for assessment: pre-treatment, post-treatment, 3 months after the end of treatment \\
& Primary outcomes: PCL-5 \\
& Secondary outcomes: PG-13, PHQ-9, GAD-7, PTGI, DEVS, BVI \\
\hline Starting date & 30 June 2016 \\
\hline Contact information & Freie Universität Berlin \\
\hline Notes & www.drks.de/drks_web/navigate.do?navigationId=trial.HTML\&TRIAL_ID=DRKS00010245 \\
\hline
\end{tabular}

\section{ISRCTN13697710}

\section{Study name}

A study of trauma-focused online guided self help versus trauma-focused cognitive behavioural therapy for post-traumatic stress disorder.

\begin{tabular}{ll}
\hline Methods & Phase III pragmatic, assessor-blind, individually randomised, controlled, non-inferiority trial \\
\hline Participants & Adults with PTSD following a single traumatic event
\end{tabular}

Interventions

\section{Experimental arm}

Online guided self-help based on TFCBT for 8 weeks

\section{Comparator arm}

Face-to-face individual TFCBT for up to 12 weeks 
ISRCTN13697710 (Continued)

Primary outcomes: CAPS-5

Secondary outcomes: IES-R, EQ-5D-5L, WSAS, PHQ-9, GAD-7, AUDIT, MSPSS, ISI, GSES, PTCI

\begin{tabular}{ll}
\hline Starting date & October 2016 \\
\hline Contact information & Ms Katy Addison \\
& rapid@cardiff.ac.uk \\
\hline Notes & www.isrctn.com/ISRCTN13697710 \\
\hline
\end{tabular}

\section{ISRCTN16806208}

\section{Study name}

A randomised controlled trial of therapist-assisted online psychological therapies for post-traumatic stress disorder.

\begin{tabular}{|c|c|}
\hline Methods & Randomised controlled trial \\
\hline Participants & Aged $\geq 18$ with PTSD resulting from traumatic events experienced in adulthood. \\
\hline \multirow[t]{5}{*}{ Interventions } & Experimental arm \\
\hline & Internet-delivered psychological therapy started immediately \\
\hline & Comparator arm \\
\hline & Internet-delivered psychological therapy started after a delay of 13 weeks \\
\hline & $\begin{array}{l}\text { Both therapies involve completing therapy modules online and assignments over } 3 \text { months, with } \\
\text { guidance via messages and weekly telephone calls with an experienced psychological therapist. }\end{array}$ \\
\hline \multirow[t]{2}{*}{ Outcomes } & $\begin{array}{l}\text { Time points for assessment: baseline; } 6 \text { weeks; end of therapy/waiting; and } 3 \text { months, } 6 \text { months, } \\
\text { and } 12 \text { months after end of therapy }\end{array}$ \\
\hline & $\begin{array}{l}\text { Primary outcome: PTSD symptoms (measured by weekly questionnaires and by interviewing par- } \\
\text { ticipants regarding their experiences) }\end{array}$ \\
\hline Starting date & January 2017 \\
\hline \multirow[t]{2}{*}{ Contact information } & Maxie Scheske \\
\hline & maxie.scheske@psy.ox.ac.uk \\
\hline Notes & $\begin{array}{l}\text { www.psy.ox.ac.uk/research/oxford-centre-for-anxiety-disorders-and-trauma/anxiety-disor- } \\
\text { ders/post-traumatic-stress-disorder }\end{array}$ \\
\hline
\end{tabular}

\section{Lehavot 2017}

\begin{tabular}{ll}
\hline Study name & Evaluation of web-based CBT for women Veterans with PTSD \\
\hline Methods & Randomised controlled trial \\
\hline Participants & Inclusion criteria: \\
& Age $\geq 18$ years \\
\hline
\end{tabular}


Lehavot 2017 (Continued)

- Female

- Veteran of the US military

- Current PTSD

- Reports routine access to computer and Internet

- Willing to provide $\geq 1$ collateral contact

- Willing to allow investigators to leave telephone messages pertaining to the study

- Willing to be audio-taped during assessments and study coach calls

Exclusion criteria:

- Actively engaged in individual therapy in the past 2 months, or receiving couple's or group therapy in the past 2 months that focuses on PTSD

- Scheduled to receive a future individual therapy appointment or future couples' or group therapy appointment focused on PTSD at a frequency of once per month or more

- Active suicidal or violent ideation within the past 2 months or on the VA 'high risk' list for imminent danger to self or others

- Moderate or extreme substance-use disorder in the past year

- Acute psychosis, psychotic episode, or psychotic disorder diagnosis within the past year

- Unstable administration schedule or dosing of any antidepressant, anxiolytic, or sedative-hypnotic

\section{Experimental arm}

DESTRESS-WV Tailored online intervention for PTSD for women Veterans with coach support.

Participants will be asked to log on to the website twice per week for about 30-60 minutes each time. On 2 occasions, participants will be asked to write about current stressors or hassles. Additionally, on 2 occasions participants will be asked to write about a traumatic experience and then rewrite it. Participants will be guided in using various coping skills taught in the program during this writing process. Homework assignments will include stress management skills that participants will be asked to practice and apply. A study coach will call each participant once a week for 8 weeks for about 15 minutes to review their progress with the program.

\section{Comparator arm}

Telephone monitoring

A study coach will call participants once a week for 8 weeks for approximately 15 minutes. The coach will assess participants' PTSD symptoms and safety. She will encourage participants to use the time on the call to discuss any current life issues or problems that they would like. Active listening and rephrasing will be used, while teaching cognitive-behavioural strategies will be avoided.

\section{Outcomes}

Time point for assessment: baseline, 8-16 weeks, 20-28 weeks, and 32-40 weeks

Primary outcomes: PCL-5

Secondary outcomes: PHQ-8, quality of life (Q-LES-Q -SF)

Starting date July 2016

\footnotetext{
Contact information
} Keren Lehavot

VA Puget Sound Health Care System Seattle Division, Seattle 
McLean 2017

Study name

Design of a randomized controlled trial examining the efficacy and biological mechanisms of webprolonged exposure and present-centered therapy for PTSD among active-duty military personnel and veterans.

\begin{tabular}{ll}
\hline Methods & Randomised controlled trial \\
\hline Participants & 120 Participants are active duty military personnel stationed at Fort Hood, TX, and veterans in the \\
surrounding area who have deployed since 11 September 2001 , who are seeking treatment for \\
PTSD, aged $18-65$ years. Up to 170 people will be consented and screened to obtain data for analy- \\
sis from 120 participants ( 60 participants in each treatment condition). \\
Inclusion criteria \\
- PTSD diagnosis, determined by CAPS- 5 clinical interview and CAPS-5 score $\geq 25$, as well as expo- \\
sure to a combat-related Criterion A event that was experienced during deployment. The diagno- \\
sis of PTSD may be indexed to the combat-related Criterion A event, or to another Criterion A event \\
- Remain in the local area for the next 3 months following the first assessment \\
Exclusion criteria \\
- Recent manic episode or psychotic disorder (determined by the bipolar and psychosis sections \\
of the MINI) \\
- Current alcohol dependence (determined by the AUDIT) \\
Evidence of moderate or severe TBI (determined by an inability to comprehend baseline screening \\
- Current suicidal ideation severe enough to warrant immediate intervention (determined by DSI- \\
SS and corroborated by a clinical risk assessment by a credentialed provider) \\
- Other psychiatric disorders severe enough to warrant designation as the primary disorder
\end{tabular}

Concomitant medications are not exclusionary; all medication changes are monitored for the duration of the trial.

Interventions Web PE versus PCT

Primary outcome: PTSD severity, as measured by CAPS-5, a structured diagnostic interview that assesses DSM-5 criteria for PTSD and yields information about PTSD symptom severity and PTSD diagnostic status.

Secondary measures include: AUDIT; CEQ; CTQ; DSI-SS; GAD-7; ISI; LEC; MINI-7.0; PCL-5, 1-month and 2-week versions; PHQ-9; PHQ-15; PTCl; PWPQ (based on the Perceptions of Computerized Therapy Questionnaire); STAXI-2; VR-12

\begin{tabular}{ll}
\hline Starting date & Unknown \\
\hline Contact information & Carmen P McLean \\
& Stanford University School of Medicine \\
& mcleanca@pennmedicine.upenn.edu \\
\hline Notes & \\
\hline
\end{tabular}


NCT02556645

Study name

A comparison of web-prolonged exposure (web-PE) and present-centred therapy (PCT) for PTSD among active-duty military personnel.

\section{Methods}

Randomised, single-blind, parallel-assigned clinical trial

\section{Participants}

Inclusion criteria:

- Adult male and female active duty military personnel or veterans who have deployment since 9/11, aged $18-65$ years seeking treatment for PTSD. Veterans must be eligible for military medical care on Fort Hood

- Diagnosis of PTSD as determined by DSM-5 (CAPS-5) clinical interview score $\geq 25$. Person has experienced a Criterion $A$ event that is a specific combat-related event or high magnitude operational experience that occurred during a period of recent military deployment. Diagnosis of PTSD may be indexed to that event or to another Criterion A event

- Able to speak and read English

- Indication that the participant plans to be in the area for the next 5 months following the first assessment

Exclusion criteria:

- Recent manic episode (past 12 months) or a psychotic disorder (as determined by the bipolar and psychosis sections of the MINI)

- Current alcohol dependence (as determined by score $\geq 4$ on items \#4-6 and a total score of $\geq 20$ on AUDIT)

- Evidence of a moderate or severe TBI (as determined by the inability to comprehend the baseline screening questionnaires)

- Current suicidal ideation severe enough to warrant immediate attention (as determined by the DSI-SS and corroborated by a clinical risk assessment by a credentialed provider)

- Other psychiatric disorders severe enough to warrant designation as the primary disorder

- Recent course of PE within the past 12 months

- Currently engaged in evidence-based treatment for PTSD (e.g. PET or CPT)

Interventions

\section{Experimental arm}

Web-PE

$10 \times 60$-minute psychotherapy sessions over 8 weeks, focused on gradually confronting distressing trauma-related memories and reminders. Web-PE is an Internet-based version of PE for PTSD

\section{Comparator arm}

\section{Therapist-delivered PCT}

$10 \times 60$-minute psychotherapy sessions over 8 weeks, focused on identifying and solving day-today problems as they are brought up by the participants

Outcomes Time point for assessment: 3 years

Primary outcomes: PTSD (CAPS), PTSD diagnosis and severity scores (measures of depression, general anxiety, anger, and PTSD-related cognitions)

Secondary outcomes: change in associated biomarkers (cortisol response to awakening, cortisol response to script-driven imagery, salivary and serum neurosteroids), ratings of use on the Web-PE program

Starting date 22 September 2015


NCT02556645 (Continued)

University of Pennsylvania

Notes NCT02556645

NCT02611843

Study name

Peer supported web-based CBT for OEF/OIF Veterans with PTSD and substance misuse.

\section{Methods}

\section{Participants}

Inclusion criteria:

- Aged $\geq 18$ years

- OEF or OIF or OND veteran

- Hazardous or harmful substance use as measured by 1 of the following: AUDIT score $\geq 7$ (for women) or $\geq 8$ (for men); DAST score $\geq 2$; have exited a controlled environment (e.g. detoxification unit, hospital, or correctional facility) within 30 days of screening AND within 30 days prior to entering the controlled environment report heavy drinking $(>3$ drinks for women or $>4$ drinks for men on $\geq 1$ days) or use of any illegal drugs or misuse of prescription drugs (use in excess of the directions or any non-medical use); determined to have diagnostic-level PTSD (1 A symptom, 1 B symptom, 3 C symptoms AND 2 D symptoms) or subthreshold PTSD (1 A symptom, 1 B symptom, 3 C symptoms OR 2 D symptoms) on CAPS

- Currently receiving primary medical care at the Syracuse VA Medical Center

Exclusion criteria:

- Plans to move out of the area within the next 3 months

- Enrolled in psychotherapy in the last 2 months focused on substance use or PTSD

- Incurred a change in dose or type of a psychiatric medication that treats PTSD or substance use in the last 2 months

- Inability to understand or provide informed consent

- Inability to use English to participate in the consent process, the computerised intervention, or the assessments

- In need of alcohol detoxification as determined by a score of $>15$ on the CIWA-Ar

- Current plan or intent to harm self

- Medical chart diagnosis of a current psychotic disorder, including schizophrenia or bipolar disorder

- Cognitive impairment specified by a medical chart diagnosis. Diagnoses include dementia and moderate-to-severe TBI. People with mild TBI were not excluded

\section{Experimental arm}

Peer-supported web CBT

Semi-structured brief sessions conducted weekly for the 12 weeks of study treatment by a certified peer support specialist. Web CBT consists of 24 brief (i.e. 20-minute) modules. Participants will be asked to complete 2 modules per week. Module topics include the connection between PTSD and substance use, motivational enhancement, relaxation, identifying, evaluating and challenging automatic thoughts, functional analyses of substance use, substance use refusal skills, communication, anger management, pain management, and insomnia.

\section{Comparator arm}

Self-managed web CBT

Self-managed web CBT consists of 24 brief (i.e. 20-minute) modules. Participants will be asked to complete 2 modules per week. Module topics include the connection between PTSD and substance 
NCT02611843 (Continued)

use, motivational enhancement, relaxation, identifying, evaluating and challenging automatic thoughts, functional analyses of substance use, substance use refusal skills, communication, anger management, pain management, and insomnia.

\begin{tabular}{ll}
\hline Outcomes & Time point for assessment: 12 weeks \\
& Primary outcomes: PCL \\
& Secondary outcomes: TLFB \\
\hline Starting date & November 2015 \\
\hline Contact information & Kyle Possemato \\
& Syracuse Veterans Affairs Medical Center \\
\hline
\end{tabular}

Notes

NCT02929979

\begin{tabular}{|c|c|}
\hline Study name & Cognitive remediation for alcohol use disorder (AUD) and posttraumatic stress disorder (PTSD). \\
\hline Methods & Randomised, triple-blind, factorial assignment controlled trial \\
\hline Participants & $\begin{array}{l}\text { Inclusion criteria: } \\
\text { - Veteran } \\
\text { - Aged } 18-65 \text { years } \\
\text { - Meet DSM-5 diagnostic criteria for current AUD } \\
\text { - Meet DSM-5 criteria for current PTSD } \\
\text { - Willing to perform daily home-based computer exercises for } 6 \text { weeks } \\
\text { Exclusion criteria: } \\
\text { - History of, or current, psychotic disorder or schizophrenia } \\
\text { - Current scheduled (i.e. daily) prescribed use of cognitive enhancers (e.g. memantine) or stimu- } \\
\text { lants (e.g. methylphenidate) that may enhance cognitive performance } \\
\text { - Current severe TBI (DoD TBI Screen } 2 \text { ) } \\
\text { - Any type of dementia (MMSE < } 24 \text { ), delirium or medical illnesses associated with potential cogni- } \\
\text { tive issues (HIV, hypothyroidism, } B_{12} \text { deficiency) } \\
\text { - Any level of mental retardation (WTAR) } \\
\text { - Limited ability to speak/read/write/understand English (WTAR) } \\
\text { - Inadequate vision or hearing } \\
\text { - Active suicidal/homicidal intent } \\
\text { - Self-report and collateral history from medical record/primary care physician/outpatient addic- } \\
\text { tion treatment team will be used as necessary to determine inclusion and exclusion. Suicidal and } \\
\text { homicidal intent will be assessed in the context of a structured clinical interview. In the unlikely } \\
\text { event that respondents endorse active intent they will be referred immediately for treatment and } \\
\text { will be excluded from the current study }\end{array}$ \\
\hline
\end{tabular}

Interventions

Experimental arm

Cognitive training

Participants complete 22.5 hours of cognitive training exercises over 6 weeks (training 5 times a week) using an app-based program, BrainHQ. Exercises will be packaged into 4 modules (attention 
skills, memory skills, executive functioning skills, cognitive control skills) comprised of 4 exercises each. All participants will progress through the same fixed schedule of modules.

\section{Comparator arm}

Placebo control

Participants play a rotating set of commercial computer games at the same dose and frequency as the cognitive training.

Outcomes

Time points for assessment: baseline, 6 weeks, and 3 months

Primary outcomes: neuropsychological assessment (WAIS-IV, TMT, AAT, HVLT, BVMT-R, WAIS-IV WMI, CPT-II, CWIT, WCST, IGT, DDT, Tower Test (DKEFS))

Secondary outcomes: alcohol use (TLFB), functional status (IPF), PTSD symptom severity (PCL-M)

\begin{tabular}{ll}
\hline Starting date & 1 January 2015 \\
\hline Contact information & Adrienne Julie Heinz \\
& VA Palo Alto Health Care System, CA \\
\hline Notes & NCT02929979 \\
\hline
\end{tabular}

\section{NCT03208738}

\section{Study name}

Pilot evaluation of the VetChange mobile app for veterans with PTSD who engage in problem drinking.

\begin{tabular}{ll}
\hline Methods & Randomised, open-label, parallel-assignment, controlled trial \\
\hline Participants & Inclusion criteria: \\
- Self-reported status as veteran \\
- Aged 18-65 years \\
- Moderate levels of non-dependent problem drinking as determined by score on AUDIT 8-25 for \\
- Den and 5-25 for women \\
QDS \\
- Willing to provide an e-mail address for reminders and incentives \\
Exclusion criteria: \\
- High alcohol problem severity
\end{tabular}

Interventions

\section{Experimental arm 1}

VetChange mobile app

\section{Experimental arm 2}

Web-based drinking AFT + VetChange mobile app + supportive accountability tool

\section{Comparator arm}

Assessment only 
NCT03208738 (Continued)

Primary outcomes: alcohol consumption (QDS), PTSD symptoms (PCL-5)

Secondary outcomes: alcohol-related problems (SIP)

\begin{tabular}{ll}
\hline Starting date & 5 July 2017 \\
\hline Contact information & Eric Kuhn, Clinical Psychologist \\
& VA Palo Alto Health Care System \\
\hline Notes & NCT03208738 \\
\hline
\end{tabular}

\section{NTR6912}

\begin{tabular}{ll}
\hline Study name & Addition of the SUPPORT Coach in PTSD treatment \\
\hline Methods & Randomised controlled trial \\
\hline Participants & Inclusion criteria: \\
- Adults aged $\geq 18$ years & Referred to a mental healthcare institute for trauma-focused psychotherapy for PTSD following \\
$\geq 1$ traumatic experiences \\
- Meet the DSM-5 diagnostic criteria for PTSD as confirmed by the CAPS-5 \\
- In possession of a mobile phone working on ANDROID/IOS operating systems \\
Exclusion criteria: \\
- Current high risk for suicide according to MINI \\
- Serious psychiatric comorbidity, i.e. psychotic illness, bipolar affective disorder, substance-relat- \\
- ed disorders, severe personality disorder, or mental retardation
\end{tabular}

\section{Experimental arm}

SUPPORT Coach, a mobile application aiming to help people better understand and self-manage their PTSD symptoms. The SUPPORT Coach includes psychoeducation, a self-test with a monitoring feature, and exercises and tools to cope with PTSD symptoms

\section{Comparator arm}

Trauma-focused therapy

\begin{tabular}{ll}
\hline Outcomes & Time points for assessment: post-treatment at the 13th session \\
& $\begin{array}{l}\text { Primary outcomes: PTSD symptoms (measured by the difference in PCL-5 score reduction (continu- } \\
\text { ous)) } \\
\text { Secondary outcomes: feasibility and acceptance of the SUPPORT Coach app }\end{array}$ \\
\hline Starting date & 15 October 2017 \\
\hline Contact information & Dr M Oiff \\
Academic Medical Center, Amsterdam \\
m.olff@amc.uva.nl
\end{tabular}


NTR6912 (Continued)

Notes

www.amc.nl/supportcoach

AAT: Approach Avoidance Tasks; AFT: Assessment and Feedback Tool; AUD: alcohol use disorder; AUDIT: Alcohol Use Disorders Identification Test; BVI: Bochumer Change Inventory; BVMT-R: Brief Visuospatial Memory Test - Revised; CAPS: Clinically Administered PTSD Scale; CBT: cognitive behavioural therapy; CEQ: Credibility/Expectancy Questionnaire; CIWA-Ar: Clinical Institute Withdrawal Assessment for Alcohol; CPT: cognitive processing therapy; CPT-II: Conners' Continuous Performance Task II; CTQ: Childhood Trauma Questionnaire; CSS: Crisis Support Scale; CWIT: Color-Word Interference Test; DAST: ; DDT: Delay Discounting Task; DES: ; DERS: Difficulties in Emotion Regulation Scale; DES: Dissociative Experiences Scale; DEVS: Distress and Endorsement Validation Scale; DKEFS: Delis-Kaplan Executive Functions System; DoD: Department of Defense; DSI-SS: Depressive Symptoms Index-Suicidality Subscale; DSM: Diagnostic and Statistical Manual of Mental Disorders; EQ-5D: EuroQol-5D; ERQ: Emotion Regulation Questionnaire; F20-F29: schizophrenia, schizotypal and delusional, and other non-mood psychotic disorders; GAD-7: Generalised Anxiety Disorder 7 Item Scale; GSES: General Self-Efficacy Scale; HVLT: Hopkins Verbal Learning Test; I-CBT: Internet-based cognitive behavioural therapy; ICD-10: International Classification of Diseases - 10th edition; IES-R: Impact of Events Scale - Revised; IGT: Iowa Gambling Task; IPF: Inventory of Psychosocial Functioning; ISI: Insomnia Severity Index; IUS-12: Intolerance of Uncertainty Scale Score; K10: Kessler 10; LEC: Life Events Checklist; MINI: Mini-International Neuropsychiatric Interview; MMSE: Mini Mental State Examination; MSPSS: Multidimensional Scale of Perceived Social Support; NEOFFI: NEO-Five Factor Inventory Neuroticism scale; OEF: Operation Enduring Freedom; OIF: Operation Iragi Freedom; PCL: PTSD Checklist; PCL-C: PTSD Checklist - Civilian; PCL-M: PTSD Checklist - Military Version; PCT: present-centred therapy; PE: prolonged exposure; PET: positron emission tomography; PG-13: Prolonged Grief Disorder-13; PHQ-9: Patient Health Questionnaire-9; PSS-I: Posttraumatic Stress Disorder Symptom Scale; PTCI: Posttraumatic Cognition Inventory; PTGI: Post Traumatic Growth Inventory; PTSD: post traumatic stress disorder; PWPQ: Perceptions of Web-PE Questionnaire; QDS: Quick Drinking Screen; Q-LES-Q-SF: Quality of Life Enjoyment and Satisfaction Questionnaire Short Form; SDS: Sheehan Disability Scale; SEQ: Service Use Questionnaire; SIP: Short Inventory of Problems; STAXI-2: State-Trait Anger Inventory-2; SWLS: Satisfaction With Life Scale; TBI: traumatic brain injury; TFCBT: individual trauma-focused cognitive behavioural therapy; TLFB: Timeline Follow-Back; TMT: trauma management therapy; VA: Veterans Affairs; VR-12: Veterans RAND 12Item Health Survey; WAIS: Wechsler Adult Intelligence Scale; WCST: Wisconsin Card Sorting Test; WHODAS-II: World Health Organization Disability Assessment Schedule; WMI: Working Memory Index; WSAS: Work and Social Adjustment Scale; WTAR: Wechsler Test of Adult Reading.

\section{DATA AND ANALYSES}

\section{Comparison 1. Internet-based cognitive and behavioural therapy (I-C/BT) versus wait list (WL)}

\begin{tabular}{|c|c|c|c|c|}
\hline Outcome or subgroup title & $\begin{array}{l}\text { No. of } \\
\text { studies }\end{array}$ & $\begin{array}{l}\text { No. of } \\
\text { partici- } \\
\text { pants }\end{array}$ & Statistical method & Effect size \\
\hline $\begin{array}{l}\text { 1.1 Severity of PTSD symptoms } \\
\text { (post-treatment) }\end{array}$ & 8 & 560 & Std. Mean Difference (IV, Random, 95\% CI) & $-0.60[-0.97,-0.24]$ \\
\hline $\begin{array}{l}\text { 1.2 Severity of PTSD symptoms } \\
\text { (follow-up }<6 \text { months) }\end{array}$ & 3 & 146 & Std. Mean Difference (IV, Random, 95\% CI) & $-0.43[-1.41,0.56]$ \\
\hline 1.3 Dropouts & 8 & 585 & Risk Ratio (M-H, Fixed, 95\% Cl) & $1.39[1.03,1.88]$ \\
\hline $\begin{array}{l}\text { 1.4 Diagnosis of PTSD after treat- } \\
\text { ment }\end{array}$ & 1 & 62 & Risk Ratio (M-H, Fixed, 95\% Cl) & $0.53[0.28,1.00]$ \\
\hline $\begin{array}{l}1.5 \text { Severity of depression (post- } \\
\text { treatment) }\end{array}$ & 5 & 425 & Std. Mean Difference (IV, Random, 95\% CI) & $-0.61[-1.17,-0.05]$ \\
\hline $\begin{array}{l}\text { 1.6 Severity of depression (fol- } \\
\text { low-up }<6 \text { months) }\end{array}$ & 1 & 42 & Mean Difference (IV, Fixed, 95\% CI) & $-8.95[-15.57,-2.33]$ \\
\hline $\begin{array}{l}\text { 1.7 Severity of anxiety symptoms } \\
\text { (post-treatment) }\end{array}$ & 4 & 305 & Std. Mean Difference (IV, Random, 95\% CI) & $-0.67[-0.98,-0.36]$ \\
\hline
\end{tabular}




\begin{tabular}{lllll}
\hline Outcome or subgroup title & $\begin{array}{l}\text { No. of } \\
\text { studies }\end{array}$ & $\begin{array}{l}\text { No. of } \\
\text { partici- } \\
\text { pants }\end{array}$ & Statistical method & Effect size \\
\hline $\begin{array}{l}1.8 \text { Severity of anxiety symptoms } \\
\text { (follow-up }<6 \text { months) }\end{array}$ & 1 & 42 & Mean Difference (IV, Fixed, 95\% Cl) & $-12.59[-20.74,-4.44]$ \\
\hline $\begin{array}{l}1.9 \text { Quality of Life (post-treat- } \\
\text { ment) }\end{array}$ & 2 & 221 & Std. Mean Difference (IV, Random, 95\% Cl) & $0.60[0.08,1.12]$ \\
\hline
\end{tabular}

Analysis 1.1. Comparison 1: Internet-based cognitive and behavioural therapy (IC/BT) versus wait list (WL), Outcome 1: Severity of PTSD symptoms (post-treatment)

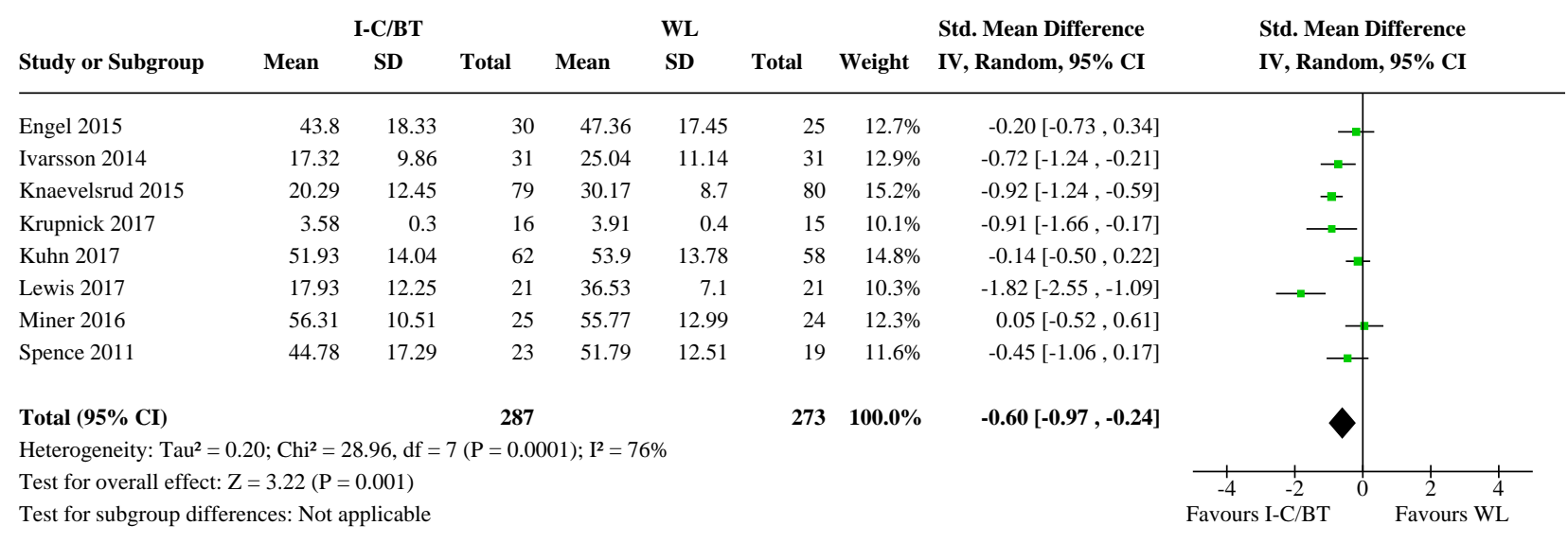

Analysis 1.2. Comparison 1: Internet-based cognitive and behavioural therapy (I-C/ BT) versus wait list (WL), Outcome 2: Severity of PTSD symptoms (follow-up $<6$ months)

\begin{tabular}{|c|c|c|c|c|c|c|c|c|c|}
\hline Study or Subgroup & Mean & $\begin{array}{l}\text { I-C/BT } \\
\text { SD }\end{array}$ & Total & Mean & $\begin{array}{l}\text { WL } \\
\text { SD }\end{array}$ & Total & Weight & $\begin{array}{l}\text { Std. Mean Difference } \\
\text { IV, Random, 95\% CI }\end{array}$ & $\begin{array}{l}\text { Std. Mean Difference } \\
\text { IV, Random, 95\% CI }\end{array}$ \\
\hline Lewis 2017 & 16.47 & 13.22 & 21 & 33.63 & 8.42 & 21 & $32.0 \%$ & $-1.52[-2.21,-0.82]$ & - \\
\hline Engel 2015 & 9.66 & 7.04 & 30 & 10.4 & 6.77 & 25 & $34.2 \%$ & $-0.11[-0.64,0.43]$ & - \\
\hline Miner 2016 & 52.01 & 14.56 & 25 & 47.87 & 13.99 & 24 & $33.8 \%$ & $0.29[-0.28,0.85]$ & \\
\hline Total $(95 \%$ CI) & & & 76 & & & 70 & $100.0 \%$ & $-0.43[-1.41,0.56]$ & \\
\hline \multicolumn{10}{|c|}{ Heterogeneity: $\mathrm{Tau}^{2}=0.66 ; \mathrm{Chi}^{2}=16.50, \mathrm{df}=2(\mathrm{P}=0.0003) ; \mathrm{I}^{2}=88 \%$} \\
\hline \multicolumn{9}{|c|}{ Test for overall effect: $Z=0.85(P=0.40)$} & $\begin{array}{cc}1 & 1 \\
-4 & -2\end{array}$ \\
\hline \multicolumn{9}{|c|}{ Test for subgroup differences: Not applicable } & Favours I-C/BT \\
\hline
\end{tabular}


Analysis 1.3. Comparison 1: Internet-based cognitive and behavioural therapy (I-C/BT) versus wait list (WL), Outcome 3: Dropouts

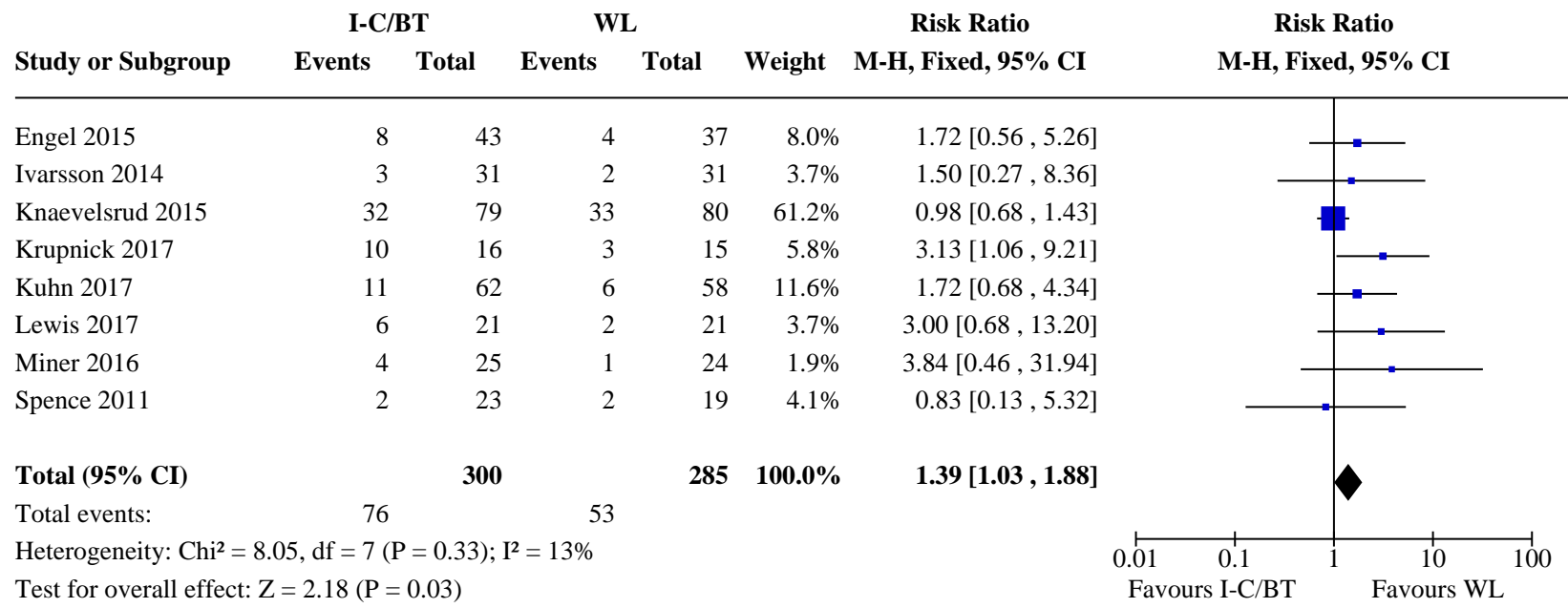

Test for subgroup differences: Not applicable Analysis 1.4. Comparison 1: Internet-based cognitive and behavioural therapy
(I-C/BT) versus wait list (WL), Outcome 4: Diagnosis of PTSD after treatment
I-C/BT
WL
Risk Ratio
Risk Ratio

Study or Subgroup Events Total Events Total Weight M-H, Fixed, 95\% CI M-H, Fixed, 95\% CI

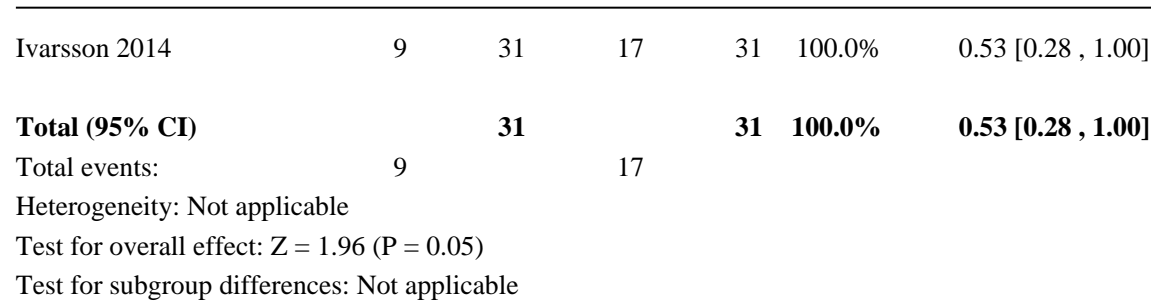

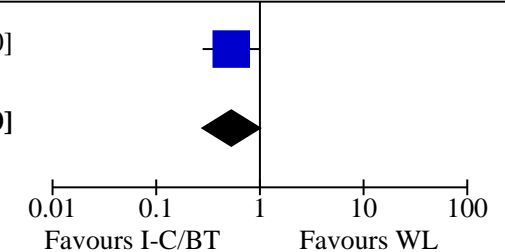

Test for subgroup differences: Not applicable

Analysis 1.5. Comparison 1: Internet-based cognitive and behavioural therapy (IC/BT) versus wait list (WL), Outcome 5: Severity of depression (post-treatment)

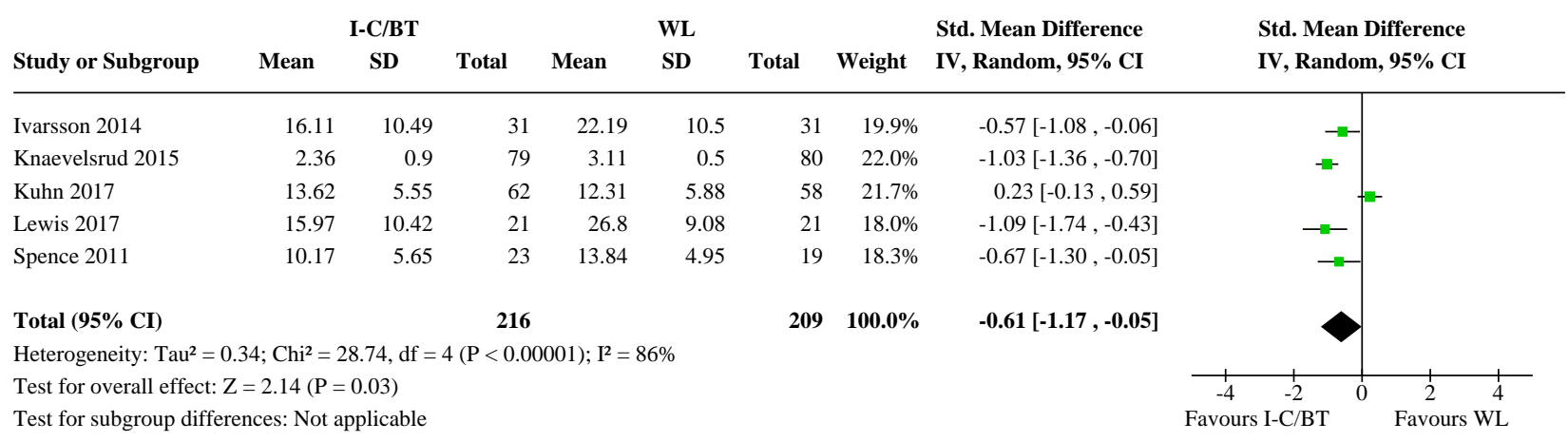


Analysis 1.6. Comparison 1: Internet-based cognitive and behavioural therapy (I-C/ BT) versus wait list (WL), Outcome 6: Severity of depression (follow-up $<6$ months)

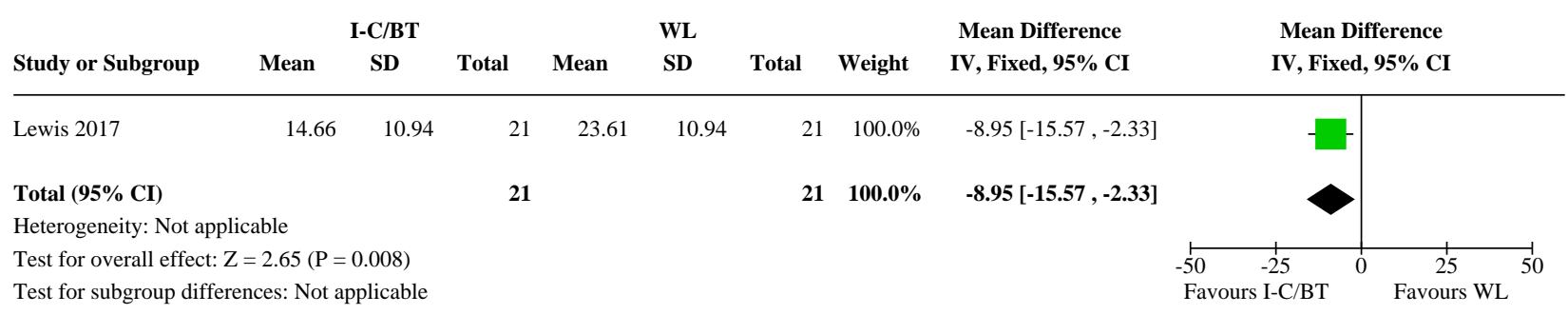

Analysis 1.7. Comparison 1: Internet-based cognitive and behavioural therapy (I-C/ BT) versus wait list (WL), Outcome 7: Severity of anxiety symptoms (post-treatment)

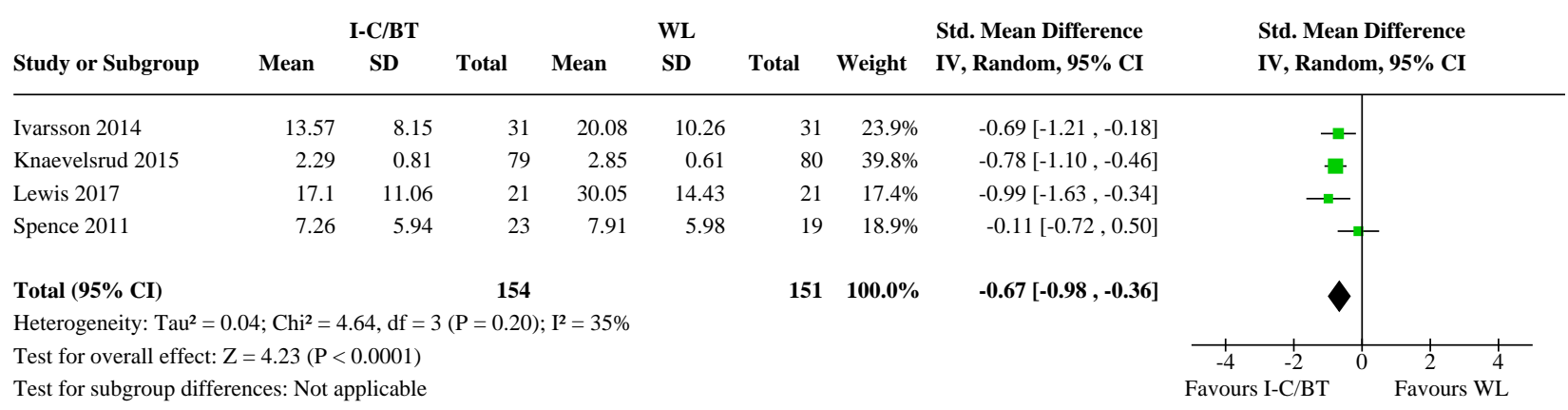

Analysis 1.8. Comparison 1: Internet-based cognitive and behavioural therapy (I-C/BT) versus wait list (WL), Outcome 8: Severity of anxiety symptoms (follow-up $<6$ months)

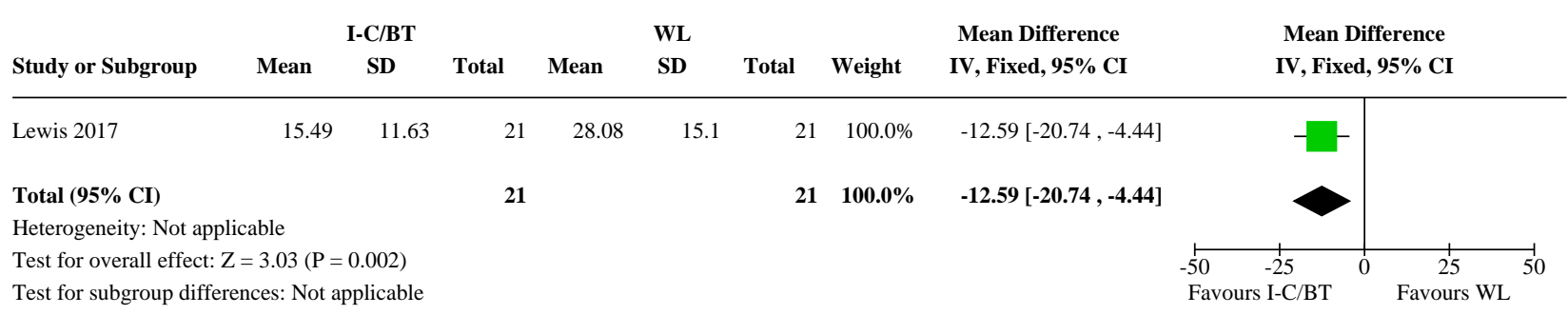

\section{Analysis 1.9. Comparison 1: Internet-based cognitive and behavioural therapy} (I-C/BT) versus wait list (WL), Outcome 9: Quality of Life (post-treatment)

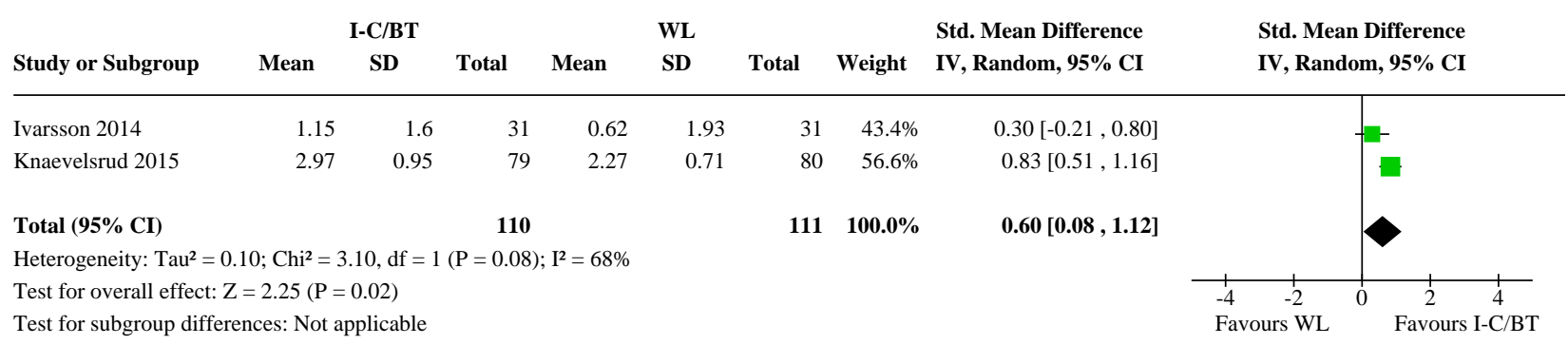


Comparison 2. Internet-based cognitive and behavioural therapy (I-C/BT) versus I-non-C/BT

\begin{tabular}{|c|c|c|c|c|}
\hline Outcome or subgroup title & $\begin{array}{l}\text { No. of } \\
\text { studies }\end{array}$ & $\begin{array}{l}\text { No. of } \\
\text { partici- } \\
\text { pants }\end{array}$ & Statistical method & Effect size \\
\hline $\begin{array}{l}\text { 2.1 Severity of PTSD symptoms } \\
\text { (post-treatment) }\end{array}$ & 2 & 82 & Std. Mean Difference (IV, Fixed, 95\% CI) & $-0.08[-0.52,0.35]$ \\
\hline $\begin{array}{l}\text { 2.2 Severity of PTSD symptoms (fol- } \\
\text { low-up }<6 \text { months) }\end{array}$ & 2 & 65 & Std. Mean Difference (IV, Fixed, 95\% CI) & $0.08[-0.41,0.57]$ \\
\hline $\begin{array}{l}2.3 \text { Severity of PTSD symptoms (fol- } \\
\text { low-up 6-12 months) }\end{array}$ & 1 & 18 & Mean Difference (IV, Fixed, 95\% CI) & $-8.83[-17.32,-0.34]$ \\
\hline 2.4 Dropouts & 2 & 132 & Risk Ratio (M-H, Fixed, 95\% Cl) & $2.14[0.97,4.73]$ \\
\hline $\begin{array}{l}\text { 2.5 Severity of depressive symptoms } \\
\text { (post-treatment) }\end{array}$ & 2 & 84 & Std. Mean Difference (IV, Random, 95\% CI) & $-0.12[-0.78,0.54]$ \\
\hline $\begin{array}{l}\text { 2.6 Severity of depressive symptoms } \\
\text { (follow-up }<6 \text { months) }\end{array}$ & 2 & 61 & Std. Mean Difference (IV, Fixed, 95\% CI) & $0.20[-0.31,0.71]$ \\
\hline $\begin{array}{l}\text { 2.7 Severity of depressive symptoms } \\
\text { (follow-up 6-12 months) }\end{array}$ & 1 & 18 & Mean Difference (IV, Fixed, 95\% CI) & $-8.34[-15.83,-0.85]$ \\
\hline $\begin{array}{l}\text { 2.8 Severity of anxiety symptoms } \\
\text { (post-treatment) }\end{array}$ & 2 & 74 & Std. Mean Difference (IV, Random, 95\% CI) & $0.08[-0.78,0.95]$ \\
\hline $\begin{array}{l}\text { 2.9 Severity of anxiety symptoms } \\
\text { (follow-up }<6 \text { months) }\end{array}$ & 2 & 60 & Std. Mean Difference (IV, Fixed, 95\% CI) & $-0.16[-0.67,0.35]$ \\
\hline $\begin{array}{l}\text { 2.10 Severity of anxiety symptoms } \\
\text { (follow-up 6-12 months) }\end{array}$ & 1 & 18 & Mean Difference (IV, Fixed, 95\% CI) & $-8.05[-15.20,-0.90]$ \\
\hline
\end{tabular}

Analysis 2.1. Comparison 2: Internet-based cognitive and behavioural therapy (IC/BT) versus I-non-C/BT, Outcome 1: Severity of PTSD symptoms (post-treatment)

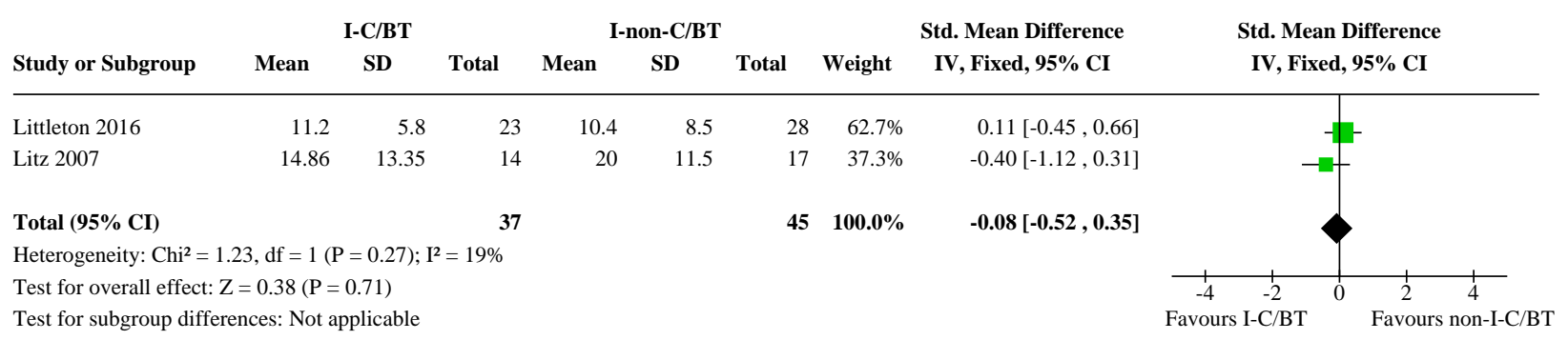


Analysis 2.2. Comparison 2: Internet-based cognitive and behavioural therapy (I-C/ BT) versus I-non-C/BT, Outcome 2: Severity of PTSD symptoms (follow-up $<6$ months)

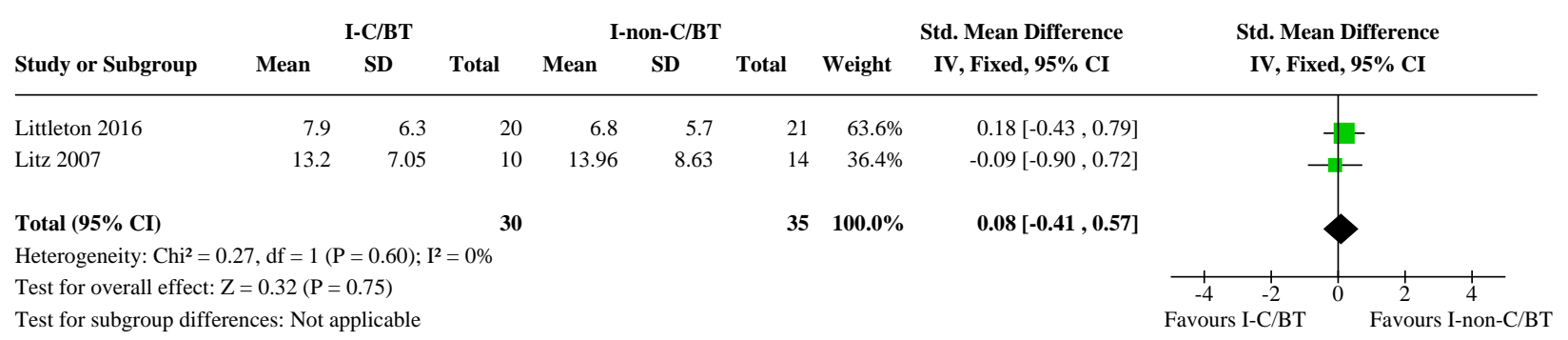

Analysis 2.3. Comparison 2: Internet-based cognitive and behavioural therapy (I-C/ BT) versus I-non-C/BT, Outcome 3: Severity of PTSD symptoms (follow-up 6-12 months)

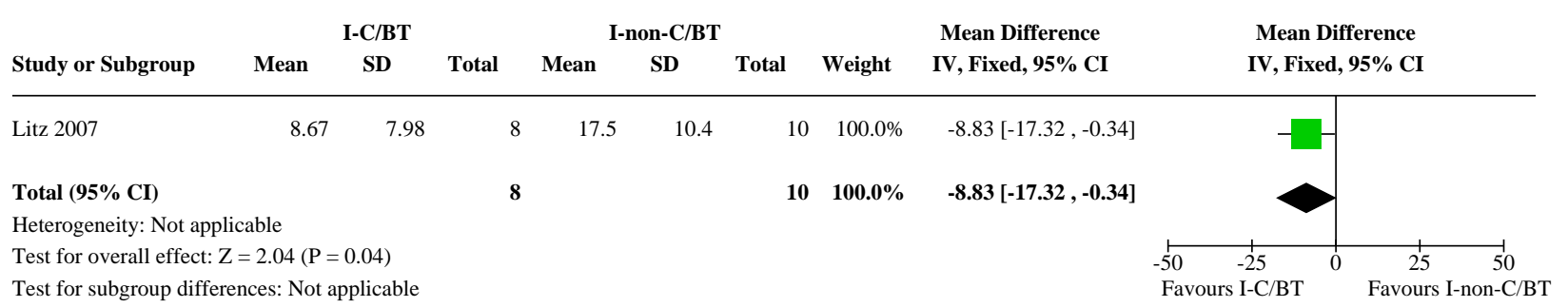

Analysis 2.4. Comparison 2: Internet-based cognitive and behavioural therapy (I-C/BT) versus I-non-C/BT, Outcome 4: Dropouts

\begin{tabular}{cccccccc} 
& \multicolumn{2}{c}{ I-C/BT } & \multicolumn{2}{c}{ I-non-C/BT } & \multicolumn{2}{c}{ Risk Ratio } & Risk Ratio \\
Study or Subgroup & Events & Total & Events & Total & Weight & M-H, Fixed, 95\% CI & M-H, Fixed, 95\% CI
\end{tabular}

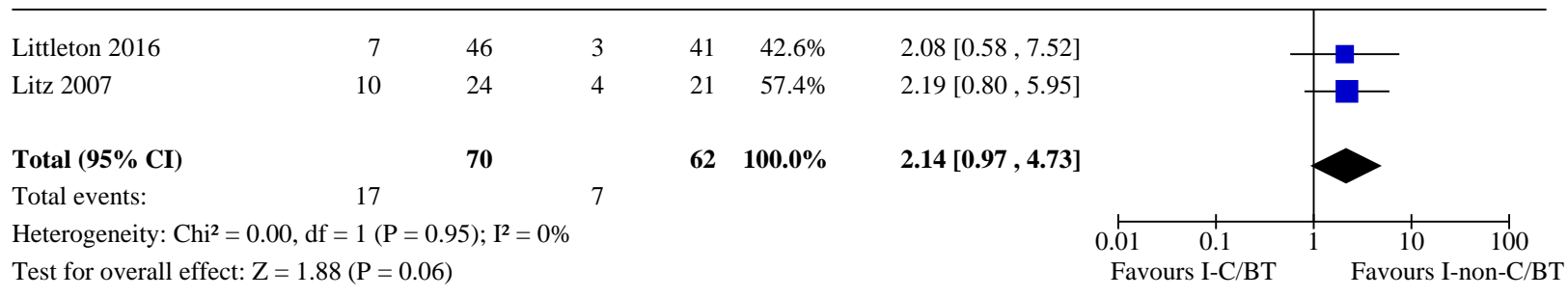

Test for subgroup differences: Not applicable

Analysis 2.5. Comparison 2: Internet-based cognitive and behavioural therapy (I-C/ BT) versus I-non-C/BT, Outcome 5: Severity of depressive symptoms (post-treatment)

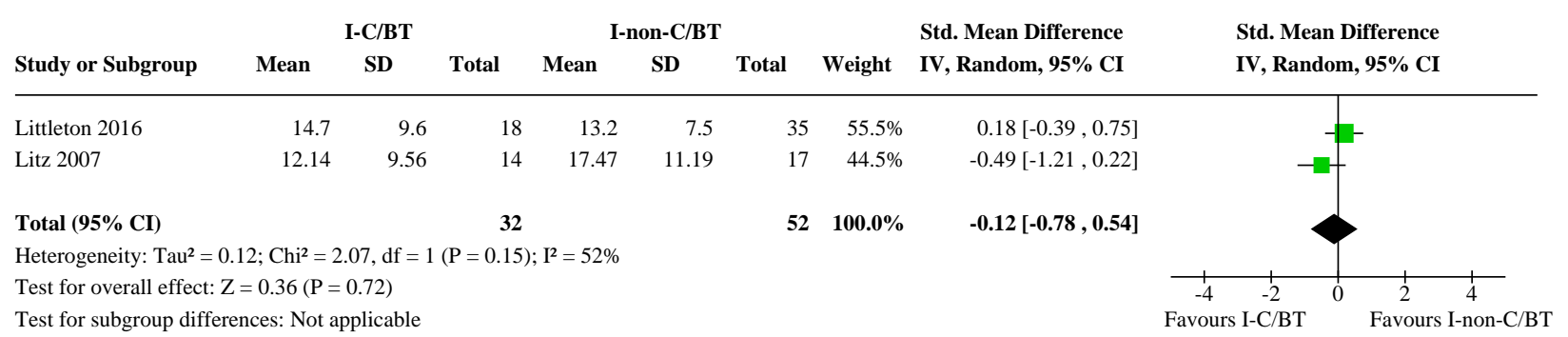


Analysis 2.6. Comparison 2: Internet-based cognitive and behavioural therapy (I-C/BT) versus I-non-C/BT, Outcome 6: Severity of depressive symptoms (follow-up $<6$ months)

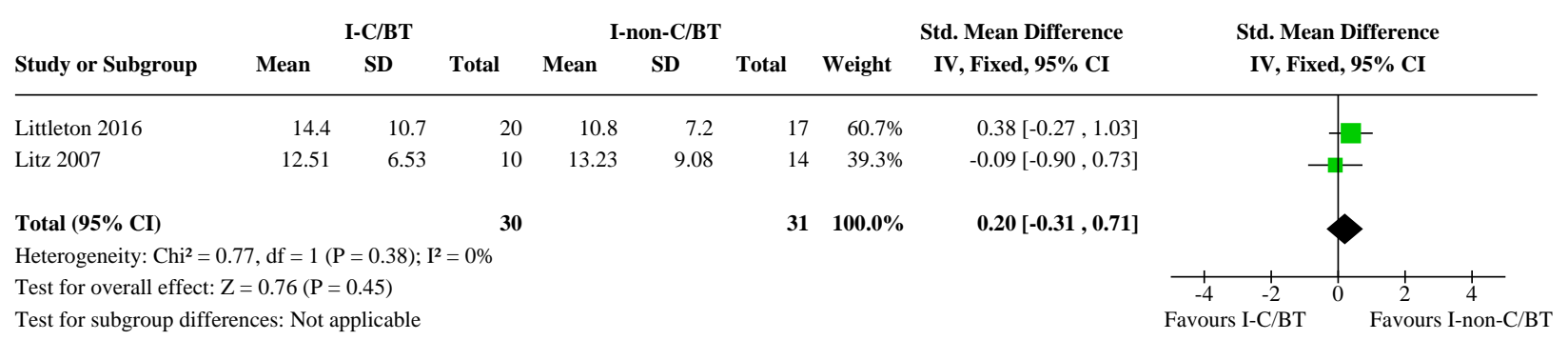

Analysis 2.7. Comparison 2: Internet-based cognitive and behavioural therapy (I-C/BT) versus I-non-C/BT, Outcome 7: Severity of depressive symptoms (follow-up 6-12 months)

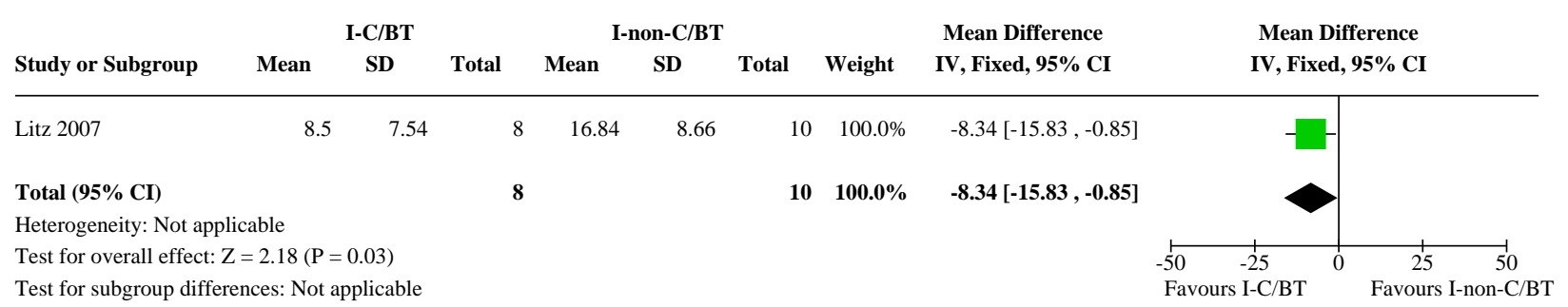

Analysis 2.8. Comparison 2: Internet-based cognitive and behavioural therapy (I-

C/BT) versus I-non-C/BT, Outcome 8: Severity of anxiety symptoms (post-treatment)

\begin{tabular}{|c|c|c|c|c|c|c|c|c|c|}
\hline \multirow[b]{2}{*}{ Study or Subgroup } & \multirow[b]{2}{*}{ Mean } & \multicolumn{2}{|c|}{ I-C/BT } & \multicolumn{3}{|c|}{ I-non-C/BT } & \multirow[b]{2}{*}{ Weight } & \multirow{2}{*}{$\begin{array}{l}\text { Std. Mean Difference } \\
\text { IV, Random, 95\% CI }\end{array}$} & \multirow{2}{*}{$\begin{array}{l}\text { Std. Mean Difference } \\
\text { IV, Random, 95\% CI }\end{array}$} \\
\hline & & SD & Total & Mean & SD & Total & & & \\
\hline Littleton 2016 & 68.9 & 23.3 & 20 & 58.7 & 16.4 & 23 & $52.3 \%$ & $0.50[-0.11,1.11]$ & - \\
\hline Litz 2007 & 8.43 & 5.93 & 14 & 12.59 & 13.45 & 17 & $47.7 \%$ & $-0.38[-1.09,0.34]$ & \\
\hline Total $(95 \%$ CI) & & & 34 & & & 40 & $100.0 \%$ & $0.08[-0.78,0.95]$ & \\
\hline \multicolumn{10}{|c|}{ Heterogeneity: $\mathrm{Tau}^{2}=0.27 ; \mathrm{Chi}^{2}=3.37, \mathrm{df}=1(\mathrm{P}=0.07) ; \mathrm{I}^{2}=70 \%$} \\
\hline \multicolumn{9}{|c|}{ Test for overall effect: $\mathrm{Z}=0.19(\mathrm{P}=0.85)$} & $\begin{array}{ll} & 1 \\
-4 & -2\end{array}$ \\
\hline \multicolumn{9}{|c|}{ Test for subgroup differences: Not applicable } & Favours I-C/BT \\
\hline
\end{tabular}

Analysis 2.9. Comparison 2: Internet-based cognitive and behavioural therapy (I-C/ BT) versus I-non-C/BT, Outcome 9: Severity of anxiety symptoms (follow-up $<6$ months)

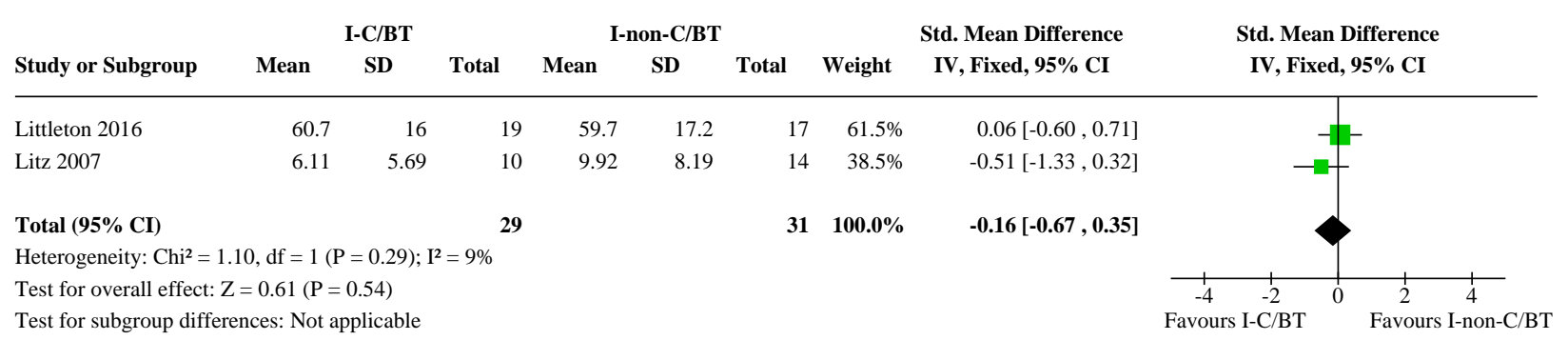


Analysis 2.10. Comparison 2: Internet-based cognitive and behavioural therapy (I-C/ BT) versus I-non-C/BT, Outcome 10: Severity of anxiety symptoms (follow-up 6-12 months)

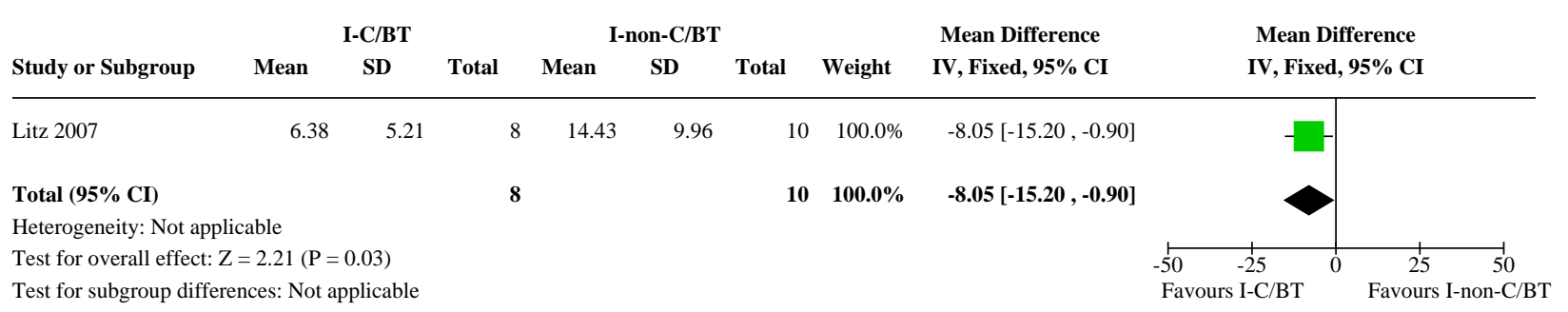

\section{APPENDICES}

\section{Appendix 1. Specialised Register - core MEDLINE search}

CCMD's core search strategy used to inform the Group's specialised register: MEDLINE Ovid A weekly search alert based on condition + RCT filter only

1. [MeSH Headings]: eating disorders/ or anorexia nervosa/ or binge-eating disorder/ or bulimia nervosa/ or female athlete triad syndrome/ or pica/ or hyperphagia/ or bulimia/ or self-injurious behavior/ or self mutilation/ or suicide/ or suicidal ideation/ or suicide, attempted/ or mood disorders/ or affective disorders, psychotic/ or bipolar disorder/ or cyclothymic disorder/ or depressive disorder/ or depression, postpartum/ or depressive disorder, major/ or depressive disorder, treatment-resistant/ or dysthymic disorder/ or seasonal affective disorder/ or neurotic disorders/ or depression/ or adjustment disorders/ or exp antidepressive agents/ or anxiety disorders/ or agoraphobia/ or neurocirculatory asthenia/ or obsessive-compulsive disorder/ or obsessive hoarding/ or panic disorder/ or phobic disorders/ or stress disorders, traumatic/ or combat disorders/ or stress disorders, post-traumatic/ or stress disorders, traumatic, acute/ or anxiety/ or anxiety, castration/ or koro/ or anxiety, separation/ or panic/ or exp anti-anxiety agents/ or somatoform disorders/ or body dysmorphic disorders/ or conversion disorder/ or hypochondriasis/ or neurasthenia/ or hysteria/ or munchausen syndrome by proxy/ or munchausen syndrome/ or fatigue syndrome, chronic/ or obsessive behavior/ or compulsive behavior/ or behavior, addictive/ or impulse control disorders/ or firesetting behavior/ or gambling/ or trichotillomania/ or stress, psychological/ or burnout, professional/ or sexual dysfunctions, psychological/ or vaginismus/ or Anhedonia/ or Affective Symptoms/ or *Mental Disorders/

2. [Title/ Author Keywords]: (eating disorder ${ }^{\star}$ or anorexia nervosa or bulimi ${ }^{\star}$ or binge eat ${ }^{\star}$ or (self adj (injur ${ }^{\star}$ or mutilat $^{\star}$ )) or suicide ${ }^{\star}$ or suicidal or parasuicid ${ }^{\star}$ or mood disorder* or affective disorder* or bipolar i or bipolar ii or (bipolar and (affective or disorder ${ }^{\star}$ )) or mania or manic or cyclothymic ${ }^{\star}$ or depression or depressive or dysthymi* or neurotic or neurosis or adjustment disorder* or antidepress ${ }^{\star}$ or anxiety disorder ${ }^{\star}$ or agoraphobia or obsess ${ }^{\star}$ or compulsi ${ }^{\star}$ or panic or phobi* or ptsd or posttrauma* or post trauma* or combat or somatoform or somati\#ation or medical ${ }^{\star}$ unexplained or body dysmorphi* or conversion disorder or hypochondria* or neurastheni* or hysteria or munchausen or chronic fatigue* or gambling or trichotillomania or vaginismus or anhedoni* or affective symptoms or mental disorder or mental health).ti,kf.

3. [RCT filter]: (controlled clinical trial.pt. or randomized controlled trial.pt. or (randomi\#ed or randomi\#ation).ab,ti. or randomly.ab. or

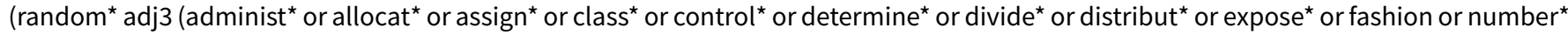
or place ${ }^{\star}$ or recruit ${ }^{\star}$ or subsitut ${ }^{\star}$ or treat $\left.{ }^{\star}\right)$ ).ab. or placebo*.ab,ti. or drug therapy.fs. or trial.ab,ti. or groups.ab. or (control* adj3 (trial ${ }^{\star}$ or study or studies)).ab,ti. or ((singl ${ }^{\star}$ or doubl* or tripl ${ }^{\star}$ or trebl ${ }^{\star}$ ) adj3 (blind ${ }^{\star}$ or mask ${ }^{\star}$ or dummy $\left.{ }^{\star}\right)$ ).mp. or clinical trial, phase ii/ or clinical trial, phase iii/ or clinical trial, phase iv/ or randomized controlled trial/ or pragmatic clinical trial/ or (quasi adj (experimental or

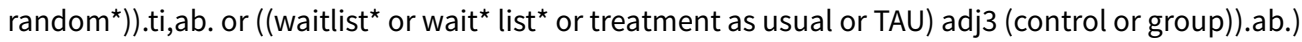

4. (1 and 2 and 3 )

Records are screened for reports of RCTs within the scope of the Cochrane Common Mental Disorders Group. Secondary reports of RCTs are tagged to the appropriate study record.

Similar weekly search alerts are also conducted on OVID Embase and PsycINFO, using relevant subject headings (controlled vocabularies) and search syntax, appropriate to each resource.

\section{Appendix 2. PILOTS: Published International Literature on Traumatic Stress - search strategy}

PILOTS was searched via ProQuest, using the following terms (RCT filter + Intervention only):

S1. su(randomized clinical trial)

S2. ti, $a b$ (randomised OR randomized OR randomisation OR randomization)

S3. ti, ab(random* NEAR/2 (allocat* OR assign* OR divid*)) 
S4. ti, ab((waitlist* OR "wait* list") NEAR/4 (control OR group))

S5. ti, ab ("treatment as usual" OR tau)

S6. ti,ab ("care as usual" OR cau)

S7. (S1 OR S2 OR S3 OR S4 OR S5 OR S6)

S8. su(self help techniques)

S9. ti,ab(self NEAR/3 (care OR change OR guid* OR help OR intervention OR manag* OR support* OR train ${ }^{\star}$ ))

S10. su(computer assisted psychotherapy)

S11. ti, ab (android OR app OR apps OR audio* OR blog OR iCBT OR i-CBT OR cCBT OR c-CBT OR cdrom OR "cell phone" OR cellphone OR chat OR computer ${ }^{\star}$ OR cyber ${ }^{\star}$ OR distance ${ }^{\star}$ OR DVD OR e-health OR eHealth OR "electronic health ${ }^{\star}$ OR e-Portal OR eportal OR eTherap* OR e-therap* OR forum* OR gaming OR "information technolog*" OR "instant messag*" OR internet* OR interapy OR ipad OR i-pad OR iphone OR ipod OR i-pod OR web* OR WWW OR "smart phone" OR smartphone OR "mobile phone" OR email* OR email ${ }^{\star}$ OR mhealth OR mhealth OR mobile OR multimedia OR multimedia OR online* OR online OR "personal digital assistant" OR PDA OR SMS OR "social medi*" OR Facebook OR software OR telecare OR telecom OR telehealth* OR telemed* OR telemonitor ${ }^{\star}$ OR telepsych*or teletherap* OR "text messag*" OR texting OR tape OR taped OR video* OR YouTube OR podcast OR virtual ${ }^{\star}$ OR remote)

S12. (S8 OR S9 OR S10 OR S11)

S13. (S7 AND S12)

[Key to database field tags: ab:abstract; ti:title; su:subject headings]

\section{Appendix 3. Other database searches}

S23 update search (March 2018)

Summary:

Ovid MEDLINE, $n=222$

Ovid Embase, $n=159$

Ovid PsycINFO, $n=79$

Proquest PILOTS, $\mathrm{n}=76$

CLib: CENTRAL, $\mathrm{n}=182$

CCMD-CTR, $\mathrm{n}=6$

Trial Registers:ICTRP, $n=58$

Trial Registers: ClinicalTrials.gov, $n=79$

Total $=861$

Duplicates removed $=359$

Records screened for study design $=502$

Non-RCTs removed $=161$

RCTs/CCTs(randomisation unclear) to screen for Population/Intervention=341

1. Ovid MEDLINE Databases: Ovid MEDLINE(R) Epub Ahead of Print, In-Process \& Other Non-Indexed Citations, Ovid MEDLINE(R) Daily and Ovid MEDLINE(R) <1946 to 1-March-2018> Search Strategy:

1 "trauma and stressor related disorders"/ or stress disorders, traumatic/ or combat disorders/ or psychological trauma/ or stress disorders, post-traumatic/ or stress disorders, traumatic, acute/ (29910)

2 (PTSD or ((posttrauma* or post-trauma* or post trauma*) adj3 (stress ${ }^{\star}$ or disorder* or psych* or symptom?)) or acute stress disorder ${ }^{\star}$ or combat disorder* or war neuros*).ti,ab,kf. (31022)

3 (((acute or traumatic) adj stress $\left.{ }^{\star}\right)$ and (expos ${ }^{\star}$ or psyc $\left.\left.{ }^{\star}\right)\right)$. ti,ab,kf. (10557)

4 (traumati\#ed adj (victim? or survivor?)).ti,ab,kf. (34)

5 (trauma* adj2 (event? or memor* or flashback* or nightmare?)).ti,ab,kf. (8079)

6 ((trauma* or posttrauma* or post-trauma* or victim* or survivor?) and (exposure adj3 (therap* or psychotherap* or training or counsel*))).ti,ab,kf. (822)

7 or/1-6 (47217)

8 (((internet or web or online) adj3 (cognitive or behavio $)$ ) or iCBT or i-CBT or ePsych* or e-Psych* or cCBT or c-CBT).ti,ab,kf. (2744)

9 (android or app or apps or blog* or CD-ROM or cell phone or cellphone or chat room or computer* or cyber* or digital or technology based or DVD or eHealth or e-health or electronic health or e-mail ${ }^{*}$ or email* or e-Portal or ePortal or eTherap* or e-therap* or forum ${ }^{\star}$ or gaming or information technolog* or instant messag* or messaging or internet* or ipad or i-pad or iphone or i-phone or ipod or i-pod or podcast or smart phone or smartphone or social network* site* or social networking or mHealth or m-health or mobile or multi-media or multimedia or online* or on-line

or personal digital assistant or PDA or SMS or social medi* or software or telecomm* or telehealth* or telemed $^{\star}$ or telemonitor $^{\star}$ or $^{\star}$

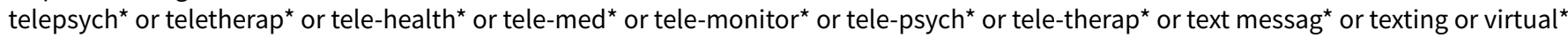
or web* or WWW).ti,ab,kf,hw. (1387965)

10 computer communication networks/ or internet/ or blogging/ or social media/ (79823)

11 cell phones/ or smartphone/ or text messaging/ or videoconferencing/ or webcasts as topic/ or wireless 
technology/ (13864)

12 (telecomm or tele-comm).ti,ab,kf. (3837)

13 Telemedicine/ (16852)

14 (eLearning or blended learning).ti,kf. (377)

15 (videoconferenc $^{\star}$ or video conferenc ${ }^{\star}$ ).ti,kf. (682)

16 (synchronous or asynchronous or (electronic adj2 deliver $\left.{ }^{\star}\right)$ ).ti,kf. (10013)

17 or/9-16 (1398330)

18 (behavio $^{\star}$ or cognitive).ti. or (psychotherap* or psychological therap* or cognitive behavio* or ((cognitive or behavio $\left.{ }^{\star}\right)$ adj2 $^{\star}$ (activat ${ }^{\star}$ or component? or defusion or modif* or restructur ${ }^{\star}$ or technique* or intervention or treatment ${ }^{\star}$ or therap $^{\star}$ or train $\left.\left.^{\star}\right)\right)$ or $\left(\left(\right.\right.$ acceptance $^{\star}$ or commitment $^{\star}$ ) adj3 therap ${ }^{\star}$ ) or rational emotive or RET or problem sol* or PST or problem focus ${ }^{\star}$ or solution focus ${ }^{\star}$ or trauma focus ${ }^{\star}$ or psychoeducat $^{\star}$ or psycho-educat ${ }^{\star}$ or psychodrama or psycho-drama* or mindfulness ${ }^{\star}$ or third wave or self-control or (self ${ }^{\star}$ adj3 (control or efficacy)) or stress manage* or exposure or reality therap* or (anxiety adj3 (management or therap* or train*)) or relaxation or guided imagery or present cent ${ }^{\star}$ or person cent ${ }^{\star}$ or person* construct $^{\star}$ or therapeutic process ${ }^{\star}$ or schema? or schemata or (thought ${ }^{\star}$ adj3 suppress $\left.{ }^{\star}\right)$ or rumination).mp. (1429420)

1917 and 18 (90124)

20 controlled clinical trial.pt. (92199)

21 randomized controlled trial.pt. (454746)

22 (randomi\#ed or randomi\#ation or randomi\#ing).ti,ab,kf. (530933)

23 (RCT or at random or (random* adj3 (assign ${ }^{\star}$ or allocat ${ }^{\star}$ or control* or crossover or cross-over or design ${ }^{\star}$ or divide $^{\star}$ or division or number))).ti,ab,kf. (417579)

24 placebo*.ab,ti,kf. (193716)

25 trial.ab,ti,kf. (497425)

26 groups.ab. (1768322)

27 (control $^{\star}$ and (trial or study or group $)$ and (placebo or waitlist ${ }^{\star}$ or wait $^{\star}$ list* or ((treatment or care) adj2 usual))).ti,ab,kf,hw. (175717)

28 ((single or double or triple or treble) adj2 (blind* or mask* or dummy)).ti,ab,kf. (155809)

29 double-blind method/ or random allocation/ or single-blind method/ (251445)

30 or/20-29 (2608017)

31 exp animals/ not humans.sh. (4430733)

3230 not 31 (2196792)

337 and (8 or 19) and $32(376)$

$34\left(2016^{\star}\right.$ or $2017^{\star}$ or $\left.2018^{\star}\right) . y r, d p, d t, e d, e p . ~(3393028)$

3533 and 34 (169)

\section{Ovid Embase < 1974 to 2018 Week 09>}

Search Strategy:

1 posttraumatic stress disorder/ (48733)

2 (PTSD or ((posttrauma* or post-trauma* or post trauma*) adj3 (stress ${ }^{\star}$ or disorder ${ }^{\star}$ or psych ${ }^{\star}$ or symptom?)) or

acute stress disorder ${ }^{\star}$ or combat disorder ${ }^{\star}$ or war neuros $\left.{ }^{\star}\right) . t i, a b, k w . ~(39840)$

3 (((acute or traumatic) adj stress $\left.{ }^{\star}\right)$ and $\left(\right.$ expos $^{\star}$ or psyc $\left.\left.c^{\star}\right)\right) . t i, a b, k w . ~(15082)$

4 (traumati\#ed adj (victim? or survivor?)).ti,ab,kw. (51)

5 (trauma* adj2 (event? or memor ${ }^{\star}$ or flashback* or nightmare?)).ti,ab,kw. (10484)

6 ((trauma* or posttrauma* or post-trauma* or victim* or survivor?) and (exposure adj3 (therap* or psychotherap* or training or counsel $\left.\left.\left.{ }^{\star}\right)\right)\right) . t i, a b, k w .(1090)$

7 or/1-6 (64330)

8 (((internet or web or online) adj3 (cognitive or behavio*)) or iCBT or i-CBT or ePsych* or e-Psych* or cCBT or c-CBT).ti,ab,kw. (3417)

9 (android or app or apps or blog* or CD-ROM or cell phone or cellphone or chat room or computer* or cyber* or digital or technology based or DVD or eHealth or e-health or electronic health or e-mail* or email* or e-Portal or ePortal or eTherap* or e-therap* or forum* or gaming or information technolog* or instant messag* or messaging or internet* or ipad or i-pad or iphone or i-phone or ipod or i-pod or podcast or smart phone or smartphone or social network* site $^{\star}$ or social networking or mHealth or m-health or mobile or multi-media or multimedia or online* or on-line

or personal digital assistant or PDA or SMS or social medi* or software or telecomm* or telehealth* or telemed ${ }^{\star}$ or telemonitor $^{\star}$ or telepsych ${ }^{\star}$ or teletherap ${ }^{\star}$ or tele-health ${ }^{\star}$ or tele-med ${ }^{\star}$ or tele-monitor ${ }^{\star}$ or tele-psych ${ }^{\star}$ or tele-therap ${ }^{\star}$

or text messag* or texting or virtual* or web* or WWW).ti,ab,kw,hw. (2257405)

10 internet/ (95360)

11 blogging/ or e-mail/ or social media/ or text messaging/ or videoconferencing/ or webcast/ or wireless communication/ (35090)

12 telecommunication/ or teleconference/ (24020)

13 telemedicine/ or telehealth/ or telepsychiatry/ or teletherapy/ (21856)

14 mobile phone/ or smartphone/ (17699)

15 mobile application.hw. (5342) 
16 *technology/ (18905)

17 computer program/ or digital computer/ or personal computer/ or computer assisted therapy/ (191199)

18 * computer/ (31065)

19 (telecomm* or tele-comm*).ti,ab,kw. (3660)

20 (eLearning or blended learning).ti,ab,kw. (1074)

21 (videoconferenc ${ }^{\star}$ or video conferenc ${ }^{\star}$ ).ti,ab,kw. (3139)

22 (synchronous or asynchronous or (electronic adj2 deliver $\left.{ }^{\star}\right)$ ).ti,ab,kw. (46708)

23 or/9-22 (2313704)

24 (behavio* or cognitive).ti. or (psychotherap* or psychological therap* or cognitive behavio* or ((cognitive or behavio*) adj2 (activat ${ }^{\star}$ or component? or defusion or modif* or restructur* or technique* or intervention or treatment*

or therap ${ }^{\star}$ or train $\left.\left.{ }^{\star}\right)\right)$ or ((acceptance ${ }^{\star}$ or commitment $\left.{ }^{\star}\right)$ adj3 therap $\left.{ }^{\star}\right)$ or rational emotive or RET or problem sol ${ }^{\star}$ or PST or problem focus ${ }^{\star}$ or solution focus ${ }^{\star}$ or trauma focus ${ }^{\star}$ or psychoeducat ${ }^{\star}$ or psycho-educat* or psychodrama or psycho-drama* or mindfulness ${ }^{\star}$ or third wave or self-control or (self* adj3 (control or efficacy)) or stress manage* or exposure or reality therap* or (anxiety adj3 (management or therap* or train*)) or relaxation or guided imagery or present cent* or person cent* or person* construct* or therapeutic process ${ }^{\star}$ or schema? or schemata or (thought* adj3 suppress ${ }^{\star}$ ) or rumination).mp. (1873339)

2523 and 24 (137132)

26 randomized controlled trial/ (488263)

27 randomization.de. (77056)

28 controlled clinical trial/ and (Disease Management or Drug Therapy or Prevention or Rehabilitation or

Therapy).fs. (251322)

29 *clinical trial/ (17644)

30 placebo.de. (319921)

31 placebo.ti,ab. (267009)

32 trial.ti. (242775)

33 (randomi\#ed or randomi\#ation or randomi\#ing).ti,ab,kw. (747796)

34 (RCT or "at random" or (random* adj3 (administ* or allocat* or assign* or class* or control* or determine* or divide* or division or distribut $^{\star}$ or expose $^{\star}$ or fashion or number ${ }^{\star}$ or place* or recruit* or subsitut ${ }^{\star}$ or treat*))).ti,ab,kw. (591088)

35 ((singl\$ or doubl\$ or trebl\$ or tripl\$) adj3 (blind\$ or mask\$ or dummy)).mp. (270404)

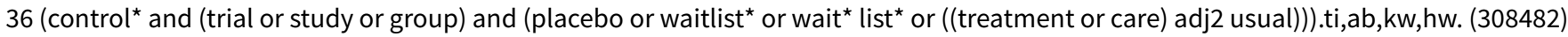

37 or/26-36 (1477215)

38 ((animal or nonhuman) not (human and (animal or nonhuman))).de. (5464926)

3937 not 38 (1348387)

407 and (8 or 25$)$ and $39(409)$

$41\left(2016^{\star}\right.$ or $2017^{\star}$ or $\left.2018^{\star}\right) \cdot y r, d c, d d .(3861880)$

4240 and 41 (159)

3. Ovid PsycINFO <1806 to February Week 4 2018>

Search Strategy:

1 posttraumatic stress disorder/ or complex ptsd/ or acute stress disorder/ or combat experience/ or "debriefing (psychological)"/ or emotional trauma/ or post-traumatic stress/ or exp stress reactions/ or traumatic neurosis/ (50805)

2 exp DISASTERS/ (8186)

3 (PTSD or ((posttrauma* or post-trauma* or post trauma*) adj3 (stress ${ }^{\star}$ or disorder* or psych* or symptom?)) or acute stress disorder ${ }^{\star}$ or combat disorder* or war neuros*).ti,ab,id. (40285)

4 (((acute or traumatic) adj stress $\left.{ }^{\star}\right)$ and $\left(\right.$ expos $^{\star}$ or psyc $\left.\left.c^{\star}\right)\right)$. ti,ab,id. (17451)

5 (traumati\#ed adj (victim? or survivor?)).ti,ab,id. (72)

6 (trauma* adj2 (event? or memor* or flashback* or nightmare?)).ti,ab,id. (12087)

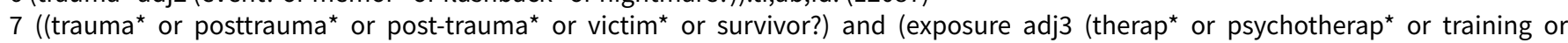
counsel $\left.\left.\left.{ }^{\star}\right)\right)\right)$. ti,ab,id,hw. (1374)

8 or/1-7 (74851)

9 (((internet or web or online) adj3 (cognitive or behavio $)$ ) or iCBT or i-CBT or ePsych* or e-Psych* or cCBT or c-CBT).ti,ab,kf. (4140)

10 (android or app or apps or blog* or CD-ROM or cell phone or cellphone or chat room or computer* or cyber* or digital or technology based or DVD or eHealth or e-health or electronic health or e-mail* or email* or e-Portal or ePortal or eTherap* or e-therap* or forum* or gaming or information technolog* or instant messag* or messaging or internet* or ipad or i-pad or iphone or i-phone or ipod or i-pod or podcast or smart phone or smartphone or social network* site* or social networking or mHealth or m-health or mobile or multi-media or multimedia or online* or on-line or personal digital assistant or PDA or SMS or social medi* or software or telecomm or telehealth* or telemed ${ }^{\star}$ or telemonitor ${ }^{\star}$ or telepsych ${ }^{\star}$ or teletherap ${ }^{\star}$ or tele-health ${ }^{\star}$ or tele-med ${ }^{\star}$ or tele-monitor ${ }^{\star}$ or tele-psych* or tele-therap* or text messag* or texting or virtual ${ }^{\star}$ or web* or WWW).ti,ab,id,hw. (307383)

11 (telecomm or tele-comm*).ti,ab,id. (2209)

12 (eLearning or blended learning).ti,ab,id. (1083) 
13 (videoconferenc* or video conferenc $\left.{ }^{\star}\right)$.ti,ab,id. (1594)

14 (synchronous or asynchronous or (electronic adj2 deliver $\left.{ }^{\star}\right)$ ).ti,ab,id. (6808)

15 internet/ or websites/ (30308)

16 mobile devices/ or cellular phones/ (5527)

17 social media/ or online social networks/or blog/ or online community/ or text messaging/ (11563)

18 electronic communication/ or exp computer mediated communication/ or electronic learning/ (9168)

19 online therapy/ or telemedicine/ (6365)

20 telecommunications media/ (1359)

21 teleconferencing/ (836)

22 technology/or information technology/ or exp computer applications/ or computer software/ (105192)

23 computers/ or computer games/ or digital computers/ or microcomputers/ (18106)

24 or/10-23 (340421)

25 (behavio* or cognitive).ti. or (psychotherap* or psychological therap* or cognitive behavio* or ((cognitive or

behavio*) adj2 (activat $^{\star}$ or component? or defusion or modif* or restructur ${ }^{\star}$ or technique ${ }^{\star}$ or intervention or treatment* ${ }^{\star}$ or therap ${ }^{\star}$ or train $\left.^{\star}\right)$ ) or ((acceptance or commitment $\left.^{\star}\right)$ adj3 therap $\left.{ }^{\star}\right)$ or rational emotive or RET or problem sol ${ }^{\star}$ or PST

or problem focus ${ }^{\star}$ or solution focus ${ }^{\star}$ or trauma focus* or psychoeducat* or psycho-educat* or psychodrama or psycho-drama* or mindfulness ${ }^{\star}$ or third wave or self-control or (self* adj3 (control or efficacy)) or stress manage* or exposure or

reality therap* or (anxiety adj3 (management or therap* or train*)) or relaxation or guided imagery or present cent ${ }^{\star}$ or person cent $^{\star}$ or person ${ }^{\star}$ construct $^{\star}$ or therapeutic process ${ }^{\star}$ or schema? or schemata or (thought* adj3 suppress*) or

rumination).ti,ab,id,hw. (795951)

26 (self adj (care or change or guide* or help or intervention or manag* or support* or train ${ }^{\star}$ )).ti,id. (12088)

2724 and (25 or 26) (56488)

28 clinical trials.sh. (10820)

29 (randomi\#ed or randomi\#ation or randomi\#ing).ti,ab,id. (72509)

30 (RCT or at random or (random* adj3 (assign* or allocat* or control* or crossover or cross-over or design* or

divide* or division or number))).ti,ab,id. (82020)

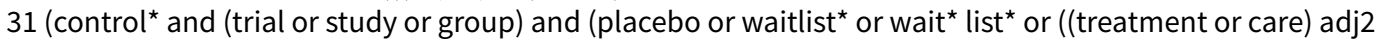

usual))).ti,ab,id,hw. (25590)

32 ((single or double or triple or treble) adj2 (blind* or mask* or dummy)).ti,ab,id. (24054)

33 trial.ti. (25583)

34 placebo.ti,ab,id,hw. (37267)

35 treatment outcome.md. (18762)

36 treatment effectiveness evaluation.sh. (21858)

37 mental health program evaluation.sh. (2028)

38 or/28-37 (169119)

398 and (9 or 27$)$ and $38(266)$

$40\left(2016^{\star}\right.$ or $2017^{\star}$ or $\left.2018^{\star}\right) \cdot y r$,an. $(411714)$

4139 and 40 (79)

\section{PILOTS: Published International Literature on Traumatic Stress}

PILOTS will be searched via ProQuest, using the following terms (RCT filter + Intervention only):

S1. su(randomized clinical trial)

S2. ti,ab(randomised OR randomized OR randomisation OR randomization)

S3. ti,ab(random* NEAR/2 (allocat ${ }^{\star}$ OR assign ${ }^{\star}$ OR divid*))

S4. ti,ab((waitlist* OR "wait* list") NEAR/4 (control OR group))

S5. ti,ab("treatment as usual" OR tau)

S6. ti,ab("care as usual" OR cau)

S7. (S1 OR S2 OR S3 OR S4 OR S5 OR S6)

$\mathrm{S} 8$. su(self help techniques)

S9. ti,ab(self NEAR/3 (care OR change OR guid* OR help OR intervention OR manag* OR support* OR train*))

S10. su(computer assisted psychotherapy)

S11. ti,ab(android OR app OR apps OR audio* OR blog OR iCBT OR i-CBT OR cCBT OR c-CBT OR cdrom OR "cell phone" OR cellphone OR chat OR computer* OR cyber* OR distance* OR DVD OR e-health OR eHealth OR "electronic health*" OR e-Portal OR eportal OR eTherap* OR e-therap* OR forum* OR gaming OR "information technolog*" OR "instant messag*" OR internet* OR interapy OR ipad OR i-pad OR iphone OR ipod OR i-pod OR web* OR WWW OR "smart phone" OR smartphone OR "mobile phone" OR email* OR email* OR mhealth OR mhealth OR mobile OR multimedia OR multimedia OR online* OR online OR "personal digital assistant" OR PDA OR SMS OR "social medi*" OR Facebook OR software OR telecare OR telecom* OR telehealth* OR telemed* OR telemonitor* OR telepsych*or teletherap* OR "text messag*" OR texting OR tape OR taped OR video* OR YouTube OR podcast OR virtual ${ }^{\star}$ OR remote)

S12. (S8 OR S9 OR S10 OR S11)

S13. (S7 AND S12)

Internet-based cognitive and behavioural therapies for post-traumatic stress disorder (PTSD) in adults (Review) 
[Key to database field tags: ab:abstract; ti:title; su:subject headings]

\section{Cochrane Central Register of Controlled Trials (CENTRAL)}

Issue 2, 2018

\#1 MESH DESCRIPTOR Stress Disorders, Post-Traumatic1318

\#2 MESH DESCRIPTOR Stress Disorders, Traumatic22

\#3 MESH DESCRIPTOR Combat Disorders97

\#4 MESH DESCRIPTOR Stress Disorders, Traumatic, Acute25

\#5 ((posttrauma* or post-trauma* or post trauma*) adj3 (stress* or disorder ${ }^{\star}$ or psych* or symptom $\left.\left.{ }^{\star}\right)\right): \mathrm{TI}, \mathrm{AB}, \mathrm{KY} 2809$

\#6 PTSD:TI,AB,KY2049

\#7 ( "acute stress disorder" or "combat disorder*" or "war neuros"):TI,AB,KY149

\#8 (\#1 OR \#2 OR \#3 OR \#4 OR \#5 OR \#6 OR \#7)3268

\#9 ((android or app or apps or audio* or blog or iCBT or CCBT or i-CBT or C-CBT or CD-ROM or ?cell phone? or cellphone or chat or computer* or cyber ${ }^{\star}$ or distance* or DVD or eHealth or e-health or "electronic health*" or e-Portal or ePortal or eTherap* or e-therap* or forum or gaming or ?information technolog*? or "instant messag*" or internet* or interapy or ipad or i-pad or iphone or i-phone or ipod or i-pod or web* $^{\star}$ or WWW or "smart phone" or smartphone or ?mobile phone? or e-mail* or email* or mHealth or m-health or mobile or multi-media or multimedia or online* or on-line or?personal digital assistant? or PDA or SMS or "social medi*" or Facebook or software or telecomm* or telehealth* or telemed ${ }^{\star}$ or telemonitor ${ }^{\star}$ or telepsych*or teletherap* or "text messag*" or texting or tape or taped or video* or YouTube or podcast or virtual* or remote)):TI,AB,KY AND 31/05/2016 TO 30/03/2018:DL26410

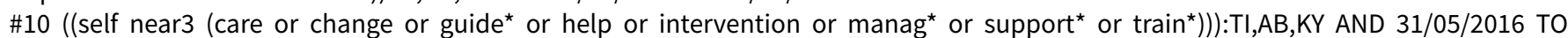
30/03/2018:DL2923

\#11 (\#9 OR \#10)28235

\#12 (\#8 AND \#11)182

\section{6a. CCMDCTR-Studies Register:}

Condition $=\left(\right.$ PTSD or *trauma* or "acute stress" or "stress reaction") AND Intervention $=$ (computer ${ }^{\star}$ or internet or web* or online or selfhelp or self-manage* or self-change)

6b. CCMDCTR-References Register was searched using a more sensitive set of terms to identify additional untagged or uncoded reports of RCTs:

\#1. (PTSD or "trauma* or "combat disorder" or "stress reaction" or "acute stress" or "stress disorder" or "war neurosis"):ab,ti,kw,ky,emt,mh,mc

\#2. (self near3 (care or change or guide* or help or intervention or manag ${ }^{\star}$ or support* or train $\left.{ }^{\star}\right)$ ):ab,ti,kw,ky,emt,mh,mc

\#3. (android or app or apps or audio* or blog or iCBT or CCBT or i-CBT or c-CBT or CD-ROM or "cell phone" or cellphone or chat or computer* or cyber ${ }^{\star}$ or distance* or DVD or eHealth or e-health or "electronic health*" or e-Portal or ePortal or eTherap* or e-therap* or forum* or gaming or "information technolog" or "instant messag*" or internet* or interapy or ipad or i-pad or iphone or i-phone or ipod or i-pod or web* or WWW or "smart phone" or smartphone or "mobile phone" or e-mail* or email* or mHealth or m-health or mobile or multi-media or multimedia or online* or on-line or "personal digital assistant" or PDA or SMS or "social medi*" or Facebook or software or telecomm* or telehealth* or telemed* or telemonitor* or telepsych*or teletherap* or "text messag*" or texting or tape or taped or video* or YouTube or podcast or virtual* or remote):ab,ti,kw,ky,emt,mh,mc

\#4. (\#1 and (\#2 or \#3)) (6)

[Key to CRS field tags: ab:abstract; ti:title; kw:CRG keywords; ky:other keywords; emt:EMTREE headings; mh:MeSH headings; mc:MeSH checkwords]

\section{International Trial Registers}

ClinicalTrials.gov (all years)

internet OR web OR online OR iCBT | PTSD OR POSTTRAUMATIC STRESS DISORDER OR POST TRAUMATIC STRESS DISORDER $n=79$

WHO International Clinical Trials Registry Platform (ICTRP

PTSD AND internet OR PTSD AND web OR PTSD AND online OR PTSD AND iCBT OR POSTTRAUMATIC STRESS DISORDER AND internet OR POSTTRAUMATIC STRESS DISORDER AND web OR POSTTRAUMATIC STRESS DISORDER AND online OR POSTTRAUMATIC STRESS DISORDER AND ICBT OR POST TRAUMATIC STRESS DISORDER AND internet OR POST TRAUMATIC STRESS DISORDER AND WEb OR POST TRAUMATIC STRESS DISORDER AND online OR POST TRAUMATIC STRESS DISORDER AND iCBT $\mathrm{n}=58$

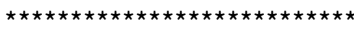

\section{WHAT'S NEW}




\begin{tabular}{lll}
\hline Date & Event & Description \\
\hline 21 May 2020 & Amended & $\begin{array}{l}\text { Clarification message from the Co-ordinating Editor added to the } \\
\text { Declarations of interest statement about the review's compli- } \\
\text { ance with the Cochrane conflict of interest policy, which includes } \\
\text { the relevant parts of the Cochrane Commercial Sponsorship Pol- } \\
\text { icy. }\end{array}$ \\
\end{tabular}

\section{HISTORY}

Protocol first published: Issue 5, 2015

Review first published: Issue 12, 2018

\section{CONTRIBUTIONS OFAUTHORS}

\begin{tabular}{|c|c|}
\hline Task & Completed by \\
\hline Drafting of the protocol & $C L, A B, N R, J B$ \\
\hline Development of a search strategy (in conjunction with CCMD's Trial Search Co-ordinator) & $\mathrm{CL}$ \\
\hline Trial selection & $\mathrm{CL}, \mathrm{AB}$, Arbitrated by NR \\
\hline Data extraction & $C L, A B$, Arbitrated by NR \\
\hline 'Risk of bias' assessment & $\mathrm{CL}, \mathrm{AB}, \mathrm{LR}$, Arbitrated by NR \\
\hline Data entry & $C L, L R$ \\
\hline Analysis & $\mathrm{CL}$ \\
\hline Interpretation of analysis & $C L, A B, N R, J B$ \\
\hline Drafting of final review & $C L, A B, N R, J B, L R$ \\
\hline Preparation of 'Summary of findings' tables & $C L, L R$ \\
\hline Checking that final review meets all mandatory MECIR standards before submission & $C L, A B, N R, J B, L R$ \\
\hline Keeping the review up-to-date & $C L, A B, N R, J B$ \\
\hline
\end{tabular}

\section{DECLARATIONS OF INTEREST}

$\mathrm{CL}$ : involved in the development and evaluation of an online, guided self-help programme (Spring: a step-by-step treatment programme for PTSD) in conjunction with the software development company Healthcare Learning Smile-on.

NR: involved in the development and evaluation of an online, guided self-help programme (Spring: a step-by-step treatment programme for PTSD) in conjunction with the software development company Healthcare Learning Smile-on.

$A B$ : none.

LR: none.

JB: involved in the development and evaluation of an online, guided self-help programme (Spring: a step-by-step treatment programme for PTSD) in conjunction with the software development company Healthcare Learning Smile-on. 
Clarification statement added from the Co-ordinating Editor on 21 May 2020: This review was found by the Cochrane Funding Arbiters, postpublication, to be noncompliant with the Cochrane conflict of interest policy, which includes the relevant parts of the Cochrane Commercial Sponsorship Policy. It will be updated within 12 months. The update will have a majority of authors and lead author free of conflicts.

\section{SOURCES OF SUPPORT}

\section{Internal sources}

- Cardiff University, Other

\section{External sources}

- No sources of support supplied

\section{DIFFERENCES BETWEEN PROTOCOLANDREVIEW}

Attrition has been removed as an outcome measure of adverse events to avoid duplication of dropout as an outcome measure.

\section{IN DEX TERMS}

\section{Medical Subject Headings (MeSH)}

Anxiety [diagnosis] [therapy]; Behavior Therapy [ ${ }^{*}$ statistics \& numerical data]; Cognitive Behavioral Therapy; Depression [diagnosis] [therapy]; Patient Dropouts [statistics \& numerical data]; Randomized Controlled Trials as Topic; Severity of Illness Index; Stress Disorders, Post-Traumatic [diagnosis] [psychology] [therapy]; Waiting Lists

\section{MeSH check words}

Adult; Humans 